\title{
Rapid Construction of Fused Heteropolycyclic Aromatics via Palladium-Catalyzed Domino Arylations of Imidazopyridine Derivatives
}

\author{
Chenwei Xue ${ }^{\dagger}$, Jianwei Han*† Min Zhao and Limin Wang*† \\ ${ }^{\dagger}$ Key Laboratory for Advanced Materials and Feringa Nobel Prize Scientist Joint Research \\ Center, Institute of Fine Chemicals, School of Chemistry and Molecular Engineering, East China \\ University of Science \& Technology, 130 Meilong Road, Shanghai, 200237, China. \\ * Shanghai-Hong Kong Joint Laboratory in Chemical Synthesis, Shanghai Institute of Organic \\ Chemistry, The Chinese Academy of Sciences, 345 Lingling Road, Shanghai 200032, China
}

Email: jianweihan@ecust.edu.cn,wanglimin@ecust.edu.cn 


\section{Table of Contents}

Part 1. General Information................................................S3

a. Methods

b. Materials

Part 2. Preparation of substituted 2-phenylimidazo[1,2-a]pyridines............S4

Part 3. Synthesis of Products from Arylation Reactions........................S4

Part 4. Synthesis of 3aa from 2-phenylimidazo[1,2-a]pyridine................S5

Part 5. Characterization data of all compounds..............................S5

Part 6. Mechanistic Studis................................................S21

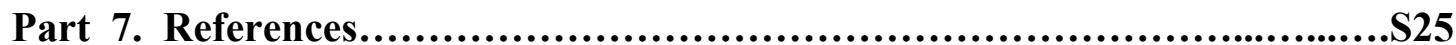

Part 8. X-Ray Diffraction Structural Determination Details..................S26

Part 9. ${ }^{1} \mathrm{H},{ }^{13} \mathrm{C}$ and ${ }^{19} \mathrm{~F}$ NMR Spectra of Products...........................S28 


\section{Part 1. General Information}

\section{a. Methods:}

NMR spectrum: ${ }^{1} \mathrm{H},{ }^{13} \mathrm{C}$, and ${ }^{19} \mathrm{~F}$ NMR spectra were recorded in $\mathrm{CDCl}_{3}$ (with tetramethylsilane as an internal standard) on a Bruker AVANCE 400 or Bruker Ascend $600(600 \mathrm{MHz})$ spectrometer at ambient temperature. Data were reported as follows: Chemical shifts $(\delta)$ are reported in ppm, coupling constants $(\mathrm{J})$ are in Hertz $(\mathrm{Hz})$. The following abbreviations were used to explain the multiplicities: $\mathrm{s}=$ singlet, $\mathrm{d}=$ doublet, $\mathrm{t}=$ triplet, $\mathrm{q}=$ quartet, $\mathrm{m}=$ multiplet, $\mathrm{br}=$ broad.

Mass spectroscopy: Mass spectra were in general recorded on a Waters LCT Premier XE spectrometer or EI Mass spectra were measured on HP HP5989A, Aglient HP5873 or Waters Micromass GCT mass spectrometer. ESI-MS analyses were performed in positive ionization mode on an Agilent 1100-MSD or Bruker Daltonics FTMS-7 mass spectrometer.

Chromatography: Column chromatography was performed with silica gel (200-300 mesh ASTM).

\section{b. Materials:}

All solvents were dried and/or distilled by standard methods. All reagents were purchased from commercial sources and used without further purification. Reactions were monitored by TLC (detection with UV light). The diaryliodonium salts were synthesized according to the literature procedures ${ }^{[1-5]}$. Substituted 2-phenylimidazo[1,2- $\left.\alpha\right]$ pyridine were prepared through the reaction of substituted 2-aminopyridine with substituted phenacyl bromide ${ }^{6}$. 
Part 2.Preparation of substituted 2-phenylimidazo[1,2- $\alpha]$ pyridines. ${ }^{6}$<smiles>Nc1c[R]ccn1</smiles>

1.25 equiv<smiles>[R][R]c1ccccc1C(=O)CBr</smiles>

1.0 equiv

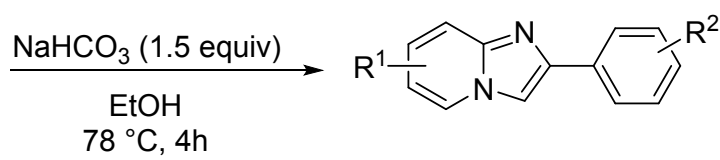

$78^{\circ} \mathrm{C}, 4 \mathrm{~h}$

To an ethanol solution containing 2-bromoacetophenones ( $10 \mathrm{mmol}, 1.0$ equiv) and 2-aminopyridines (12.5 mmol, 1.25 equiv) was added $\mathrm{NaHCO}_{3}(15 \mathrm{mmol}, 1.5$ equiv). The reaction mixture was stirred at $78^{\circ} \mathrm{C}$ for 4 hours. After completion of the reaction, the solvent was evaporated under vacuum and DCM $(20 \mathrm{~mL})$ was added. Then the mixture was diluted with saturated sodium bicarbonate solution $(20 \mathrm{~mL})$, the organic layer was separated and the water phase was washed with DCM $(20 \mathrm{~mL})$, the combined organic layer was evaporated under vacuum and dried with anhydrous $\mathrm{NaSO}_{4}$. The crude products were purified using flash column chromatography (Petroleum ether/EtOAc, 2/1) on silica gel to afford the desired substituted 2-phenylimidazo[1,2- $\alpha]$ pyridines.

\section{Part 3.Synthesis of Products from Arylation Reactions.}

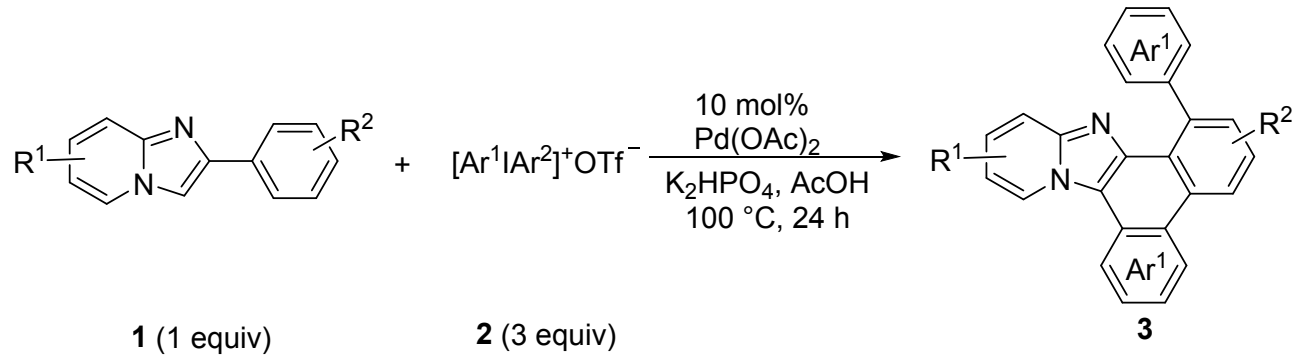

General procedure: To an oven-dried Schlenk tube was added imidazopyridine 1 (1 mmol, 1 equiv.), diaryliodonium salts 2 (3 mmol, 3 equiv.), $\mathrm{Pd}(\mathrm{OAc})_{2}(10 \mathrm{~mol} \%)$ and $\mathrm{K}_{2} \mathrm{HPO}_{4}(2 \mathrm{mmol}, 2$ equiv.). Acetic acid $(10 \mathrm{~mL})$ was added via syringe, and the mixture was stirred at $100{ }^{\circ} \mathrm{C}$ in an oil bath. After TLC indicated that the 2-aryl imidazopyridine was completely consumed. The solvent was washed with saturated sodium bicarbonate solution $(30 \mathrm{~mL})$ and extracted with DCM $(15 \mathrm{~mL})$ for three times. Then the solvent was evaporated under vacuum. The crude products were purified using flash column chromatography (eluent: Petroleum ether/EtOAc or Petroleum ether/DCM ) on silica gel to afford the desired product 3 . 
Part 4.Synthesis of 3aa from 2-phenylimidazo[1,2- $\alpha]$ pyridine.
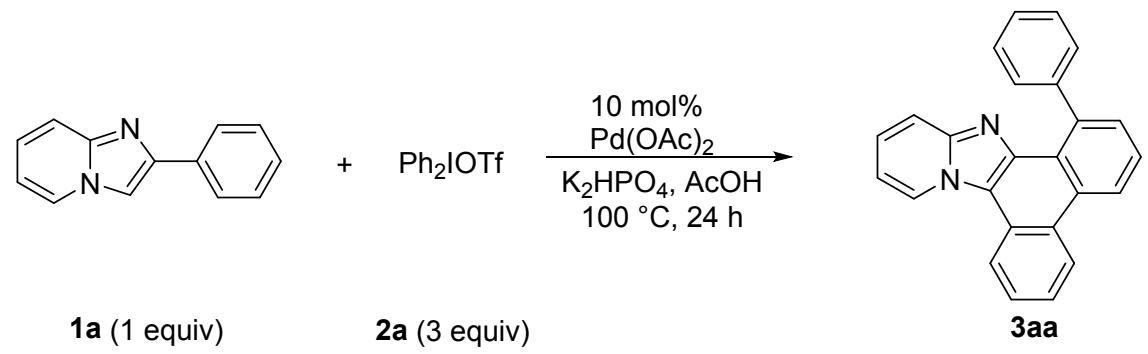

To an oven-dried Schlenk tube was added 2-phenylimidazo[1,2- $\alpha]$ pyridine 1a (1 mmol, 1 equiv.), $\mathrm{Ph}_{2} \mathrm{IOTf} 2 \mathrm{a}$ ( 3 mmol, 3 equiv.), $\mathrm{Pd}(\mathrm{OAc})_{2}\left(10 \mathrm{~mol} \%\right.$ ) and $\mathrm{K}_{2} \mathrm{HPO}_{4}$ (2 mmol, 2 equiv.). Acetic acid (10 $\mathrm{mL}$ ) was added via syringe, and the mixture was stirred at $100^{\circ} \mathrm{C}$ in an oil bath. After TLC indicated that the 2-phenylimidazo[1,2- $\alpha]$ pyridine was completely consumed. The solvent was washed with saturated sodium bicarbonate solution $(30 \mathrm{~mL})$ and extracted with DCM $(15 \mathrm{~mL})$ for three times. Then the solvent was evaporated under vacuum. The crude products were purified using flash column chromatography (eluent: Petroleum ether/EtOAc, 5/1) on silica gel to afford the desired product 3aa $275.5 \mathrm{mg}(80 \%)$.

Part 5. Characterization data of all compounds.

\section{8-Phenylphenanthro $\left[9^{\prime}, 10^{\prime}: 4,5\right]$ imidazo[1,2- $\left.\alpha\right]$ pyridine (3aa)}

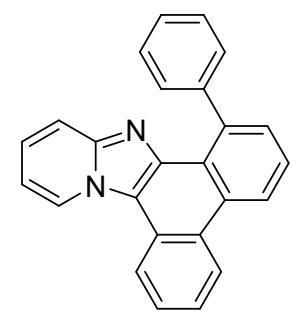

Following the general procedure, the reaction of $1 \mathbf{a}(38.8 \mathrm{mg}, 0.2 \mathrm{mmol})$, iodonium salts $2 \mathbf{a}$ ( $258 \mathrm{mg}, 0.6$ $\mathrm{mmol}), \mathrm{Pd}(\mathrm{OAc})_{2}(4.5 \mathrm{mg}, 10 \mathrm{~mol} \%), \mathrm{K}_{2} \mathrm{HPO}_{4}\left(69.7 \mathrm{mg}, 0.4 \mathrm{mmol}\right.$.) and acetic acid $(4 \mathrm{~mL})$ at $100{ }^{\circ} \mathrm{C}$ for $24 \mathrm{~h}$. The product was purified by flash chromatography (Petroleum ether/EtOAc, 5/1) to give 3aa $57.2 \mathrm{mg}(83 \%)$ as a yellow solid: M. p.: $121-123{ }^{\circ} \mathrm{C} . \mathbf{~}^{\mathbf{H}} \mathbf{H} \mathbf{N M R}\left(\mathbf{4 0 0} \mathbf{M H z}, \mathbf{C D C l}_{3}\right) \delta 9.11(\mathrm{~d}, J=7.1 \mathrm{~Hz}$, $1 \mathrm{H}), 8.92(\mathrm{~d}, J=8.3 \mathrm{~Hz}, 1 \mathrm{H}), 8.81(\mathrm{~d}, J=8.2 \mathrm{~Hz}, 1 \mathrm{H}), 8.53(\mathrm{~d}, J=8.1 \mathrm{~Hz}, 1 \mathrm{H}), 7.77(\mathrm{t}, J=7.7 \mathrm{~Hz}, 1 \mathrm{H})$, $7.75-7.70(\mathrm{~m}, 1 \mathrm{H}), 7.67(\mathrm{t}, J=7.4 \mathrm{~Hz}, 1 \mathrm{H}), 7.61(\mathrm{~d}, J=6.7 \mathrm{~Hz}, 1 \mathrm{H}), 7.57$ (ddd, $J=14.4,8.4,6.1 \mathrm{~Hz}$, 3H), $7.52-7.44$ (m, 3H), 7.26 (dd, $J=8.9,6.4 \mathrm{~Hz}, 1 \mathrm{H}), 6.96$ (t, $J=6.4 \mathrm{~Hz}, 1 \mathrm{H}) .{ }^{13} \mathbf{C}$ NMR (101 MHz, $\left.\mathbf{C D C l}_{3}\right)_{\delta} \delta 146.05,144.07,141.13,140.48,130.91,130.82,129.97,128.59,127.43,127.41,126.57$, 125.95, 125.93, 125.10, 125.01, 124.97, 124.74, 123.76, 122.70, 120.15, 119.52, 119.11, 112.20. HRMS $\mathbf{m} / \mathbf{z}$ (EI): calculated for $\mathrm{C}_{25} \mathrm{H}_{16} \mathrm{~N}_{2}[\mathrm{M}]^{+} 344.1313$, found 344.1292 . 


\section{6-Fluoro-8-phenylphenanthro[9',10':4,5]imidazo[1,2- $\alpha]$ pyridine (3ab)}

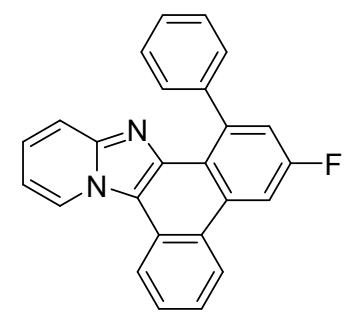

Following the general procedure, the reaction of $\mathbf{1 b}(42.4 \mathrm{mg}, 0.2 \mathrm{mmol})$, iodonium salts $\mathbf{2 a}(258 \mathrm{mg}$, $0.6 \mathrm{mmol}), \mathrm{Pd}(\mathrm{OAc})_{2}(4.5 \mathrm{mg}, 10 \mathrm{~mol} \%), \mathrm{K}_{2} \mathrm{HPO}_{4}(69.7 \mathrm{mg}, 0.4 \mathrm{mmol}$.) and acetic acid (4 mL) at 100 ${ }^{\circ} \mathrm{C}$ for $24 \mathrm{~h}$. The product was purified by flash chromatography (Petroleum ether/EtOAc, 5/1) to give 3ab $49.3 \mathrm{mg}(68 \%)$ as a yellow solid: M. p.: $213-215{ }^{\circ} \mathrm{C} .{ }^{1} \mathbf{H}$ NMR (400 MHz, $\left.\mathbf{C D C l}_{3}\right) \delta 9.09(\mathrm{~d}, J=7.1 \mathrm{~Hz}$, $1 \mathrm{H}), 8.76(\mathrm{~d}, J=8.2 \mathrm{~Hz}, 1 \mathrm{H}), 8.51(\mathrm{~d}, J=8.2 \mathrm{~Hz}, 1 \mathrm{H}), 8.41(\mathrm{dd}, J=11.2,2.5 \mathrm{~Hz}, 1 \mathrm{H}), 7.78(\mathrm{dd}, J=$ 11.2, 4.1 Hz, 1H), $7.65(\mathrm{dd}, J=11.3,4.1 \mathrm{~Hz}, 1 \mathrm{H}), 7.58-7.46(\mathrm{~m}, 6 \mathrm{H}), 7.37(\mathrm{dd}, J=8.8,2.5 \mathrm{~Hz}, 1 \mathrm{H})$, 7.27 (ddd, $J=9.0,6.6,1.0 \mathrm{~Hz}, 2 \mathrm{H}), 6.97(\mathrm{td}, J=7.0,1.2 \mathrm{~Hz}, 1 \mathrm{H}) .{ }^{13} \mathbf{C}$ NMR (101 MHz, CDCl $\left.\mathbf{3}\right) \delta 160.68$ $(\mathrm{d}, J=246.4 \mathrm{~Hz}), 146.10,143.15(\mathrm{~d}, J=8.4 \mathrm{~Hz}), 142.92(\mathrm{~d}, J=1.7 \mathrm{~Hz}), 140.95,132.66(\mathrm{~d}, J=8.3 \mathrm{~Hz})$, $129.72,128.01,127.80(\mathrm{~d}, J=3.7 \mathrm{~Hz}), 127.52,127.02,125.90,125.23,125.15,124.73,124.10$, $121.70,119.57,119.05,118.98(\mathrm{~d}, J=23.2 \mathrm{~Hz}), 112.27,108.22,108.00 .{ }^{19} \mathbf{F} \mathbf{N M R}\left(376 \mathrm{MHz}, \mathrm{CDCl}_{3}\right)$ $\delta$-114.95 (s). HRMS m/z (EI): calculated for $\mathrm{C}_{25} \mathrm{H}_{15} \mathrm{FN}_{2}[\mathrm{M}]^{+} 362.1219$, found 362.1212 .

\section{6-Chloro-8-phenylphenanthro $\left[9^{\prime}, 1^{\prime}: 4,5\right]$ imidazo $[1,2-\alpha]$ pyridine (3ac)}

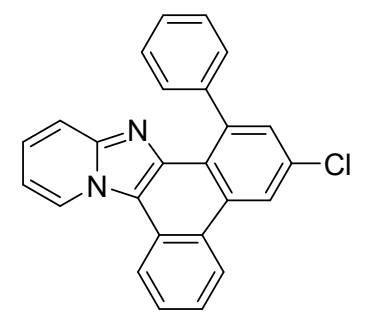

Following the general procedure, the reaction of $1 \mathbf{c}(45.7 \mathrm{mg}, 0.2 \mathrm{mmol})$, iodonium salts $\mathbf{2 a}(258 \mathrm{mg}, 0.6$ mmol), $\mathrm{Pd}(\mathrm{OAc})_{2}(4.5 \mathrm{mg}, 10 \mathrm{~mol} \%), \mathrm{K}_{2} \mathrm{HPO}_{4}\left(69.7 \mathrm{mg}, 0.4 \mathrm{mmol}\right.$.) and acetic acid $(4 \mathrm{~mL})$ at $100{ }^{\circ} \mathrm{C}$ for $24 \mathrm{~h}$. The product was purified by flash chromatography (Petroleum ether/EtOAc, 5/1) to give 3ac $49.2 \mathrm{mg}$ (65\%) as a yellow solid: M. p.: 254-257 ${ }^{\circ} \mathrm{C} .{ }^{1} \mathbf{H}$ NMR (400 MHz, $\left.\mathbf{C D C l}_{3}\right) \delta 9.05(\mathrm{~d}, J=7.1 \mathrm{~Hz}$, $1 \mathrm{H}), 8.78(\mathrm{~d}, J=8.3 \mathrm{~Hz}, 1 \mathrm{H}), 8.72(\mathrm{~d}, J=1.9 \mathrm{~Hz}, 1 \mathrm{H}), 8.47(\mathrm{~d}, J=8.1 \mathrm{~Hz}, 1 \mathrm{H}), 7.76(\mathrm{t}, J=7.6 \mathrm{~Hz}, 1 \mathrm{H})$, $7.63(\mathrm{t}, J=7.7 \mathrm{~Hz}, 1 \mathrm{H}), 7.58(\mathrm{~d}, J=2.0 \mathrm{~Hz}, 1 \mathrm{H}), 7.53(\mathrm{q}, J=3.4 \mathrm{~Hz}, 3 \mathrm{H}), 7.49(\mathrm{dd}, J=7.4,4.3 \mathrm{~Hz}, 4 \mathrm{H})$, $7.30-7.23(\mathrm{~m}, 1 \mathrm{H}), 6.99-6.94(\mathrm{~m}, 1 \mathrm{H}) .{ }^{13} \mathbf{C} \mathbf{N M R}\left(\mathbf{1 0 1} \mathbf{M H z}, \mathbf{C D C l}_{\mathbf{3}}\right) \delta 146.21,142.76,142.38$, $140.59,132.20,132.01,130.87,129.90,128.13,127.68,127.63,127.18,126.05,125.58,125.16,125.04$, 124.09, 123.44, 122.46, 120.28, 119.67, 119.20, 112.56. HRMS m/z (EI): calculated for $\mathrm{C}_{25} \mathrm{H}_{15} \mathrm{ClN}_{2}$ $[\mathrm{M}]^{+}$378.0924, found 378.0917. 


\section{6-Bromo-8-phenylphenanthro $\left[9^{\prime}, 10^{\prime}: 4,5\right]$ imidazo $[1,2-\alpha]$ pyridine (3ad)}

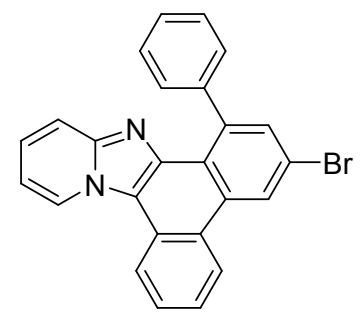

Following the general procedure, the reaction of $\mathbf{1 d}(54.6 \mathrm{mg}, 0.2 \mathrm{mmol})$, iodonium salts $2 \mathbf{a}(258 \mathrm{mg}$, $0.6 \mathrm{mmol}), \mathrm{Pd}(\mathrm{OAc})_{2}(4.5 \mathrm{mg}, 10 \mathrm{~mol} \%), \mathrm{K}_{2} \mathrm{HPO}_{4}(69.7 \mathrm{mg}, 0.4 \mathrm{mmol}$.) and acetic acid (4 mL) at 100 ${ }^{\circ} \mathrm{C}$ for $24 \mathrm{~h}$. The product was purified by flash chromatography (Petroleum ether/EtOAc, 5/1) to give 3ad $46.6 \mathrm{mg}(55 \%)$ as a yellow solid: M. p.: 256-258 ${ }^{\circ} \mathrm{C} .{ }^{1} \mathbf{H}$ NMR (400 MHz, $\left.\mathbf{C D C l}_{3}\right) \delta 9.09(\mathrm{~d}, J=7.1 \mathrm{~Hz}$, $1 \mathrm{H}), 8.91(\mathrm{~d}, J=1.8 \mathrm{~Hz}, 1 \mathrm{H}), 8.82(\mathrm{~d}, J=8.3 \mathrm{~Hz}, 1 \mathrm{H}), 8.51(\mathrm{~d}, J=8.1 \mathrm{~Hz}, 1 \mathrm{H}), 7.79(\mathrm{t}, J=7.6 \mathrm{~Hz}, 1 \mathrm{H})$, $7.72(\mathrm{~d}, J=1.9 \mathrm{~Hz}, 1 \mathrm{H}), 7.67$ (t, $J=7.7 \mathrm{~Hz}, 1 \mathrm{H}), 7.56-7.50(\mathrm{~m}, 3 \mathrm{H}), 7.50-7.45(\mathrm{~m}, 3 \mathrm{H}), 7.29-7.23$ $(\mathrm{m}, 1 \mathrm{H}), 6.98(\mathrm{dd}, J=9.8,3.8 \mathrm{~Hz}, 1 \mathrm{H}) .{ }^{13} \mathbf{C} \mathbf{N M R}\left(\mathbf{1 0 1} \mathbf{M H z}, \mathbf{C D C l}_{3}\right) \delta 146.23,142.67,142.47,140.61$, $133.53,132.44$, 129.91, 128.16, 127.69, 127.55, 127.19, 126.06, 125.62, 125.57, 125.18, 125.09, 124.07, 123.77, 120.35, 120.32, 119.68, 119.23, 112.60. HRMS m/z (EI): calculated for $\mathrm{C}_{25} \mathrm{H}_{15} \mathrm{BrN}_{2}[\mathrm{M}]^{+}$ 422.0419, found 422.0413 .

\section{6-Methyl-8-phenylphenanthro $\left[9^{\prime}, 10^{\prime}: 4,5\right]$ imidazo $[1,2-\alpha]$ pyridine (3ae)}

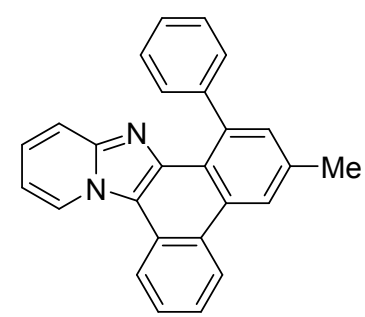

Following the general procedure, the reaction of $1 \mathrm{e}(41.6 \mathrm{mg}, 0.2 \mathrm{mmol})$, iodonium salts $2 \mathrm{a}(258 \mathrm{mg}, 0.6$ $\mathrm{mmol}), \mathrm{Pd}(\mathrm{OAc})_{2}(4.5 \mathrm{mg}, 10 \mathrm{~mol} \%), \mathrm{K}_{2} \mathrm{HPO}_{4}\left(69.7 \mathrm{mg}, 0.4 \mathrm{mmol}\right.$.) and acetic acid $(4 \mathrm{~mL})$ at $100{ }^{\circ} \mathrm{C}$ for $24 \mathrm{~h}$. The product was purified by flash chromatography (Petroleum ether/EtOAc, 5/1) to give 3ae $45.2 \mathrm{mg}$ (63\%) as a yellow solid: M. p.: $222-225{ }^{\circ} \mathrm{C} .{ }^{1} \mathbf{H}$ NMR $\left(400 \mathbf{~ M H z}, \mathbf{C D C l}_{3}\right) \delta 9.10(\mathrm{~d}, J=7.1 \mathrm{~Hz}$, $1 \mathrm{H}), 8.92(\mathrm{~d}, J=8.1 \mathrm{~Hz}, 1 \mathrm{H}), 8.60(\mathrm{~s}, 1 \mathrm{H}), 8.51(\mathrm{~d}, J=7.6 \mathrm{~Hz}, 1 \mathrm{H}), 7.80-7.71(\mathrm{~m}, 1 \mathrm{H}), 7.69-7.61(\mathrm{~m}$, 1H), $7.60-7.57(\mathrm{~m}, 1 \mathrm{H}), 7.55$ (dd, $J=9.1,5.5 \mathrm{~Hz}, 2 \mathrm{H}), 7.47$ (ddd, $J=7.8,3.8,1.5 \mathrm{~Hz}, 4 \mathrm{H}), 7.30-7.22$ (m, 1H), 6.95 (d, $J=1.1 \mathrm{~Hz}, 1 \mathrm{H}), 2.68$ (s, 3H). ${ }^{13} \mathbf{C}$ NMR (101 MHz, $\left.\mathbf{C D C l}_{3}\right) \delta 146.16,144.17,141.35$, 140.48, 135.61, 132.62, 131.07, 130.07, 128.49, 127.51, 127.37, 126.63, 125.99, 125.06, 124.65, 123.97, 122.86, 122.82, 119.80, 119.58, 119.09, 117.59, 112.17, 22.08. HRMS m/z (EI): calculated for $\mathrm{C}_{26} \mathrm{H}_{18} \mathrm{~N}_{2}$ $[\mathrm{M}]^{+}$358.1470, found 358.1464 . 


\section{6-Methoxy-8-phenylphenanthro[9',10':4,5]imidazo[1,2- $\alpha]$ pyridine (3af)}

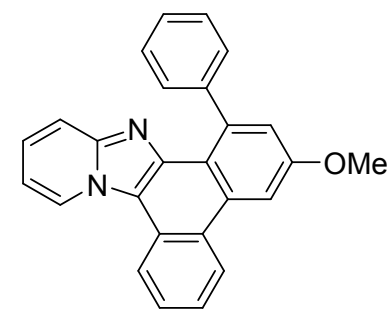

Following the general procedure, the reaction of $\mathbf{1 f}(44.8 \mathrm{mg}, 0.2 \mathrm{mmol})$, iodonium salts $\mathbf{2 a}(258 \mathrm{mg}, 0.6$ mmol), $\mathrm{Pd}(\mathrm{OAc})_{2}(4.5 \mathrm{mg}, 10 \mathrm{~mol} \%), \mathrm{K}_{2} \mathrm{HPO}_{4}\left(69.7 \mathrm{mg}, 0.4 \mathrm{mmol}\right.$.) and acetic acid $(4 \mathrm{~mL})$ at $100{ }^{\circ} \mathrm{C}$ for $24 \mathrm{~h}$. The product was purified by flash chromatography (Petroleum ether/EtOAc, 5/1) to give 3af $43.4 \mathrm{mg}(58 \%)$ as a yellow solid: M. p.: $193-195{ }^{\circ} \mathrm{C} .{ }^{1} \mathbf{H}$ NMR (400 MHz, $\left.\mathbf{C D C l}_{3}\right) \delta 9.07(\mathrm{~d}, J=7.0 \mathrm{~Hz}$, $1 \mathrm{H}), 8.82(\mathrm{~d}, J=8.3 \mathrm{~Hz}, 1 \mathrm{H}), 8.49(\mathrm{~d}, J=8.1 \mathrm{~Hz}, 1 \mathrm{H}), 8.22(\mathrm{~d}, J=2.3 \mathrm{~Hz}, 1 \mathrm{H}), 7.75(\mathrm{t}, J=7.5 \mathrm{~Hz}, 1 \mathrm{H})$, $7.63(\mathrm{t}, J=7.7 \mathrm{~Hz}, 1 \mathrm{H}), 7.58(\mathrm{dd}, J=6.4,2.9 \mathrm{~Hz}, 2 \mathrm{H}), 7.50(\mathrm{dd}, J=9.4,5.6 \mathrm{~Hz}, 4 \mathrm{H}), 7.26-7.21(\mathrm{~m}$, 1H), $6.94(\mathrm{t}, J=6.6 \mathrm{~Hz}, 1 \mathrm{H}), 4.06$ (s, 3H). ${ }^{13} \mathbf{C}$ NMR (101 MHz, $\left.\mathbf{C D C l}_{3}\right) \delta 157.55,146.18,143.85$, $142.33,141.52$, 132.55, 129.96, 128.17, 127.61, 127.54, 126.83, 125.92, 125.12, 124.96, 124.51, 124.23, 119.61, 119.46, 119.19, 119.07, 118.96, 112.08, 105.96, 55.70. HRMS m/z (EI): calculated for $\mathrm{C}_{26} \mathrm{H}_{18} \mathrm{~N}_{2} \mathrm{O}[\mathrm{M}]^{+}$374.1419, found 374.1413.

\section{6-(tert-Butyl)-8-phenylphenanthro $\left[9^{\prime}, 10^{\prime}: 4,5\right]$ imidazo $[1,2-\alpha]$ pyridine (3ag)}

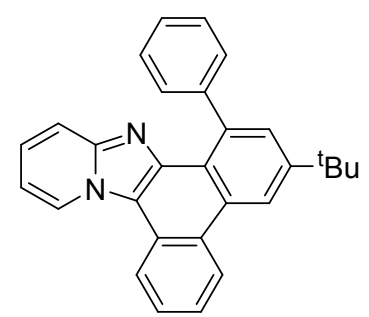

Following the general procedure, the reaction of $\mathbf{1 g}(50.1 \mathrm{mg}, 0.2 \mathrm{mmol})$, iodonium salts $\mathbf{2 a}(258 \mathrm{mg}, 0.6$ $\mathrm{mmol}), \mathrm{Pd}(\mathrm{OAc})_{2}(4.5 \mathrm{mg}, 10 \mathrm{~mol} \%), \mathrm{K}_{2} \mathrm{HPO}_{4}\left(69.7 \mathrm{mg}, 0.4 \mathrm{mmol}\right.$.) and acetic acid $(4 \mathrm{~mL})$ at $100{ }^{\circ} \mathrm{C}$ for $24 \mathrm{~h}$. The product was purified by flash chromatography (Petroleum ether/EtOAc, 5/1) to give $\mathbf{3 a g}$ $32.8 \mathrm{mg}(41 \%)$ as a yellow solid: M. p.: $169-171^{\circ} \mathrm{C} .{ }^{1} \mathbf{H}$ NMR (400 MHz, $\left.\mathbf{C D C l}_{3}\right) \delta 9.09(\mathrm{~d}, J=7.1 \mathrm{~Hz}$, $1 \mathrm{H}), 8.98(\mathrm{~d}, J=8.2 \mathrm{~Hz}, 1 \mathrm{H}), 8.86(\mathrm{~d}, J=1.6 \mathrm{~Hz}, 1 \mathrm{H}), 8.51(\mathrm{~d}, J=8.0 \mathrm{~Hz}, 1 \mathrm{H}), 7.75(\mathrm{t}, J=7.4 \mathrm{~Hz}, 1 \mathrm{H})$, $7.72(\mathrm{~d}, J=1.7 \mathrm{~Hz}, 1 \mathrm{H}), 7.68(\mathrm{t}, J=7.6 \mathrm{~Hz}, 1 \mathrm{H}), 7.64(\mathrm{dd}, J=7.3,1.9 \mathrm{~Hz}, 2 \mathrm{H}), 7.58-7.51(\mathrm{~m}, 4 \mathrm{H})$, $7.28-7.22(\mathrm{~m}, 1 \mathrm{H}), 6.94(\mathrm{t}, J=6.7 \mathrm{~Hz}, 1 \mathrm{H}), 1.59(\mathrm{~s}, 9 \mathrm{H}) .{ }^{13} \mathbf{C}$ NMR (101 MHz, CDCl $) \delta 148.62$, 146.14, 144.53, 141.30, 140.22, 130.58, 130.21, 129.21, 128.87, 127.48, 127.28, 126.63, 125.99, 124.97, 124.62, 123.97, 122.89, 119.91, 119.62, 119.11, 118.87, 112.10, 35.35, 31.70. HRMS m/z (EI): calculated for $\mathrm{C}_{29} \mathrm{H}_{24} \mathrm{~N}_{2}[\mathrm{M}]^{+} 400.1939$, found 400.1936 . 


\section{6,8-Diphenylphenanthro[9',10':4,5]imidazo[1,2- $\alpha]$ pyridine (3ah)}

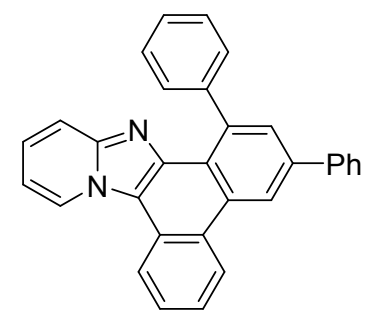

Following the general procedure, the reaction of $\mathbf{1 h}(54.1 \mathrm{mg}, 0.2 \mathrm{mmol})$, iodonium salts $\mathbf{2 a}(258 \mathrm{mg}$, $0.6 \mathrm{mmol}), \mathrm{Pd}(\mathrm{OAc})_{2}(4.5 \mathrm{mg}, 10 \mathrm{~mol} \%), \mathrm{K}_{2} \mathrm{HPO}_{4}(69.7 \mathrm{mg}, 0.4 \mathrm{mmol}$.) and acetic acid (4 mL) at 100 ${ }^{\circ} \mathrm{C}$ for $24 \mathrm{~h}$. The product was purified by flash chromatography (Petroleum ether/EtOAc, 5/1) to give 3ah $65.6 \mathrm{mg}(78 \%)$ as a yellow solid: M. p.: $233-236^{\circ} \mathrm{C} .{ }^{1} \mathbf{H}$ NMR (400 MHz, $\left.\mathbf{C D C l}_{3}\right) \delta 9.10(\mathrm{~d}, J=7.1 \mathrm{~Hz}$, $1 \mathrm{H}), 9.00(\mathrm{~d}, J=8.5 \mathrm{~Hz}, 2 \mathrm{H}), 8.52(\mathrm{~d}, J=8.0 \mathrm{~Hz}, 1 \mathrm{H}), 7.87(\mathrm{~d}, J=8.6 \mathrm{~Hz}, 3 \mathrm{H}), 7.77(\mathrm{t}, J=7.5 \mathrm{~Hz}, 1 \mathrm{H})$, $7.70-7.63(\mathrm{~m}, 3 \mathrm{H}), 7.58-7.50(\mathrm{~m}, 6 \mathrm{H}), 7.43(\mathrm{t}, J=7.4 \mathrm{~Hz}, 1 \mathrm{H}), 7.30-7.24(\mathrm{~m}, 1 \mathrm{H}), 6.97(\mathrm{t}, J=6.8$ $\mathrm{Hz}, 1 \mathrm{H}) .{ }^{13} \mathbf{C}$ NMR (101 MHz, $\mathbf{C D C l}_{3}$ ) $\delta$ 146.29, 144.07, 141.25, 141.15, 141.08, 138.58, 131.30, 130.15, 130.12 , 129.07, 128.75, 127.70, 127.65, 127.61, 127.57, 126.80, 126.03, 125.19, 125.13, 124.84, 124.18, 124.09, 121.28, 120.33, 119.69, 119.22, 112.30. HRMS m/z (EI): calculated for $\mathrm{C}_{31} \mathrm{H}_{20} \mathrm{~N}_{2}[\mathrm{M}]^{+}$ 420.1626 , found 420.1623 .

\section{8-Phenyl-6-(trifluoromethyl)phenanthro $\left[9^{\prime}, 10^{\prime}: 4,5\right]$ imidazo $[1,2-\alpha]$ pyridine (3ai)}

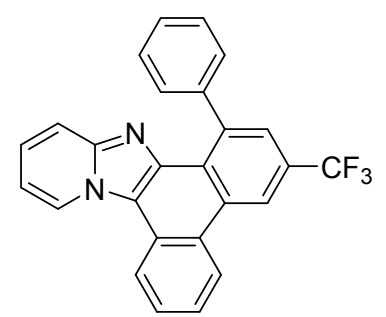

Following the general procedure, the reaction of $\mathbf{1 i}(52.4 \mathrm{mg}, 0.2 \mathrm{mmol})$, iodonium salts $\mathbf{2 a}(258 \mathrm{mg}, 0.6$ $\mathrm{mmol}), \mathrm{Pd}(\mathrm{OAc})_{2}(4.5 \mathrm{mg}, 10 \mathrm{~mol} \%), \mathrm{K}_{2} \mathrm{HPO}_{4}\left(69.7 \mathrm{mg}, 0.4 \mathrm{mmol}\right.$.) and acetic acid $(4 \mathrm{~mL})$ at $100{ }^{\circ} \mathrm{C}$ for $24 \mathrm{~h}$. The product was purified by flash chromatography (Petroleum ether/EtOAc, 5/1) to give 3ai $57.7 \mathrm{mg}(70 \%)$ as a yellow solid: M. p.: $232-234{ }^{\circ} \mathrm{C} .{ }^{1} \mathbf{H}$ NMR (400 MHz, $\left.\mathbf{C D C l}_{3}\right) \delta 9.04(\mathrm{~d}, J=9.4 \mathrm{~Hz}$, $2 \mathrm{H}), 8.88(\mathrm{~d}, J=8.2 \mathrm{~Hz}, 1 \mathrm{H}), 8.47(\mathrm{~d}, J=7.9 \mathrm{~Hz}, 1 \mathrm{H}), 7.82(\mathrm{~d}, J=1.1 \mathrm{~Hz}, 1 \mathrm{H}), 7.80-7.74(\mathrm{~m}, 1 \mathrm{H})$, $7.70-7.64(\mathrm{~m}, 1 \mathrm{H}), 7.56(\mathrm{dt}, J=6.6,3.2 \mathrm{~Hz}, 2 \mathrm{H}), 7.52(\mathrm{dd}, J=6.4,3.5 \mathrm{~Hz}, 3 \mathrm{H}), 7.32-7.25(\mathrm{~m}, 1 \mathrm{H})$, $6.97(\mathrm{td}, J=7.0,1.1 \mathrm{~Hz}, 1 \mathrm{H}) .{ }^{13} \mathbf{C}$ NMR (101 MHz, $\left.\mathbf{C D C l}_{3}\right) \delta 146.30,142.98,141.56,140.34,130.58$, $129.89,128.23,128.05,127.69,127.37,127.28,127.22,126.61$ (q, $J=3.0 \mathrm{~Hz}), 126.08,125.68,125.22$, 125.07, 124.73124 .03 (q, $J=273.7 \mathrm{~Hz}), 121.26,119.89$ (q, $J=4.0 \mathrm{~Hz}), 119.70,119.38,112.65 .{ }^{19} \mathbf{F}$ NMR (376 MHz, $\mathbf{C D C l}_{3}$ ) $\boldsymbol{\delta}$-61.67 (s). HRMS m/z (EI): calculated for $\mathrm{C}_{26} \mathrm{H}_{15} \mathrm{~F}_{3} \mathrm{~N}_{2}[\mathrm{M}]^{+} 412.1187$, found 412.1180 . 


\section{1-Methyl-8-phenylphenanthro[9',10':4,5]imidazo[1,2- $\alpha]$ pyridine (3aj)}

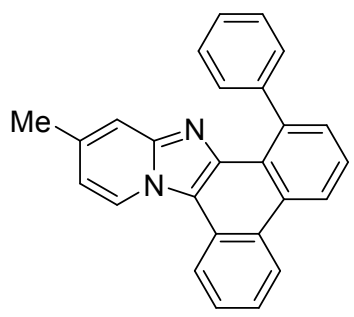

Following the general procedure, the reaction of $\mathbf{1 j}(41.6 \mathrm{mg}, 0.2 \mathrm{mmol})$, iodonium salts $\mathbf{2 a}(258 \mathrm{mg}, 0.6$ $\mathrm{mmol}), \mathrm{Pd}(\mathrm{OAc})_{2}(4.5 \mathrm{mg}, 10 \mathrm{~mol} \%), \mathrm{K}_{2} \mathrm{HPO}_{4}\left(69.7 \mathrm{mg}, 0.4 \mathrm{mmol}\right.$.) and acetic acid $(4 \mathrm{~mL})$ at $100{ }^{\circ} \mathrm{C}$ for $24 \mathrm{~h}$. The product was purified by flash chromatography (Petroleum ether/EtOAc, 5/1) to give 3aj $49.5 \mathrm{mg}(69 \%)$ as a yellow solid: M. p.: $223-225{ }^{\circ} \mathrm{C} .{ }^{1} \mathbf{H}$ NMR (400 MHz, $\left.\mathbf{C D C l}_{3}\right) \delta 8.93(\mathrm{~d}, J=7.2 \mathrm{~Hz}$, $1 \mathrm{H}), 8.90(\mathrm{~d}, J=8.4 \mathrm{~Hz}, 1 \mathrm{H}), 8.80(\mathrm{~d}, J=8.2 \mathrm{~Hz}, 1 \mathrm{H}), 8.45(\mathrm{~d}, J=8.1 \mathrm{~Hz}, 1 \mathrm{H}), 7.72$ (dt, $J=12.3,7.7$ $\mathrm{Hz}, 2 \mathrm{H}), 7.66-7.57$ (m, 4H), 7.50 (dd, $J=5.0,1.9 \mathrm{~Hz}, 3 \mathrm{H}), 7.29$ (s, 1H), 6.75 (dd, $J=7.2,1.7 \mathrm{~Hz}, 1 \mathrm{H})$, 2.38 (s, 3H). ${ }^{13} \mathbf{C}$ NMR (101 MHz, $\left.\mathbf{C D C l}_{3}\right) \delta$ 146.68, 144.28, 141.17, 140.45, 136.32, 130.90, 130.78, $130.11,128.38,127.47,127.41,126.60,125.82,125.20,125.09$, 125.03, 124.49, 123.84, 122.77, 119.96, 119.43, 117.42, 114.91, 21.31. HRMS m/z (EI): calculated for $\mathrm{C}_{26} \mathrm{H}_{18} \mathrm{~N}_{2}[\mathrm{M}]^{+} 358.1470$, found 358.1462.

\section{1-Methoxy-8-phenylphenanthro[9',10':4,5]imidazo[1,2- $\alpha]$ pyridine (3ak)}<smiles>COc1ccn2c(c1)nc1c3c(-c4ccccc4)cccc3c3ccccc3c12</smiles>

Following the general procedure, the reaction of $\mathbf{1 k}(44.8 \mathrm{mg}, 0.2 \mathrm{mmol})$, iodonium salts $\mathbf{2 a}(258 \mathrm{mg}$, $0.6 \mathrm{mmol}), \mathrm{Pd}(\mathrm{OAc})_{2}(4.5 \mathrm{mg}, 10 \mathrm{~mol} \%), \mathrm{K}_{2} \mathrm{HPO}_{4}(69.7 \mathrm{mg}, 0.4 \mathrm{mmol}$.) and acetic acid (4 mL) at 100 ${ }^{\circ} \mathrm{C}$ for $24 \mathrm{~h}$. The product was purified by flash chromatography (Petroleum ether/EtOAc, 5/1) to give 3ak $42.7 \mathrm{mg}(57 \%)$ as a yellow solid: M. p.: $229-232{ }^{\circ} \mathrm{C} .{ }^{1} \mathbf{H}$ NMR (400 MHz, $\left.\mathbf{C D C l}_{3}\right) \delta 8.94-8.88(\mathrm{~m}$, $2 \mathrm{H}), 8.81(\mathrm{~d}, J=8.1 \mathrm{~Hz}, 1 \mathrm{H}), 8.41(\mathrm{~d}, J=8.0 \mathrm{~Hz}, 1 \mathrm{H}), 7.76-7.68(\mathrm{~m}, 2 \mathrm{H}), 7.65-7.57(\mathrm{~m}, 4 \mathrm{H}), 7.54-$ $7.47(\mathrm{~m}, 3 \mathrm{H}), 6.75(\mathrm{~d}, J=2.6 \mathrm{~Hz}, 1 \mathrm{H}), 6.65(\mathrm{dd}, J=7.6,2.6 \mathrm{~Hz}, 1 \mathrm{H}), 3.84(\mathrm{~s}, 3 \mathrm{H}) .{ }^{13} \mathbf{C}$ NMR (101 MHz, $\left.\mathbf{C D C l}_{3}\right) \delta 157.96,148.11,144.35,141.39,140.32,130.87,130.59,130.07,128.13,127.46,127.40$, 126.56, 126.51, 125.63, 125.05, 124.93, 124.29, 123.63, 122.75, 119.82, 119.01, 107.35, 95.94, 55.67. HRMS m/z (EI): calculated for $\mathrm{C}_{26} \mathrm{H}_{18} \mathrm{~N}_{2} \mathrm{O}[\mathrm{M}]^{+} 374.1419$, found 374.1413. 
<smiles></smiles>

Following the general procedure, the reaction of $\mathbf{1 l}(52.4 \mathrm{mg}, 0.2 \mathrm{mmol})$, iodonium salts $\mathbf{2 a}(258 \mathrm{mg}, 0.6$ mmol), $\mathrm{Pd}(\mathrm{OAc})_{2}(4.5 \mathrm{mg}, 10 \mathrm{~mol} \%), \mathrm{K}_{2} \mathrm{HPO}_{4}\left(69.7 \mathrm{mg}, 0.4 \mathrm{mmol}\right.$.) and acetic acid $(4 \mathrm{~mL})$ at $100{ }^{\circ} \mathrm{C}$ for $24 \mathrm{~h}$. The product was purified by flash chromatography (Petroleum ether/EtOAc, 5/1) to give 3al $42.9 \mathrm{mg}(52 \%)$ as a yellow solid: M. p.: $221-223{ }^{\circ} \mathrm{C} .{ }^{1} \mathbf{H}$ NMR (400 MHz, $\left.\mathbf{C D C l}_{3}\right) \delta 9.01(\mathrm{~d}, J=7.4 \mathrm{~Hz}$, $1 \mathrm{H}), 8.82$ (d, $J=7.9 \mathrm{~Hz}, 1 \mathrm{H}), 8.72(\mathrm{~d}, J=8.0 \mathrm{~Hz}, 1 \mathrm{H}), 8.33$ (d, $J=7.8 \mathrm{~Hz}, 1 \mathrm{H}), 7.81(\mathrm{~s}, 1 \mathrm{H}), 7.75-7.60$ $(\mathrm{m}, 4 \mathrm{H}), 7.57-7.51(\mathrm{~m}, 5 \mathrm{H}), 7.04(\mathrm{dd}, J=7.3,1.8 \mathrm{~Hz}, 1 \mathrm{H}) .{ }^{\mathbf{1 3}} \mathbf{C}$ NMR (101 MHz, CDCl$)_{3} \delta 143.94$, 143.83 , 142.51, 140.83, 131.16, 131.00, 129.89, 129.18, 127.63, 127.61, 126.87, 126.62, 126.55, 126.29, $125.68,125.13,124.61,123.40(\mathrm{q}, J=272.7 \mathrm{~Hz}), 123.12,122.79,120.83,119.73,116.95(\mathrm{q}, J=5.0 \mathrm{~Hz})$ , 107.75 (q, $J=3.0 \mathrm{~Hz}) .{ }^{19} \mathbf{F}$ NMR (376 MHz, $\left.\mathbf{C D C l}_{3}\right) \delta-63.43$ (s). HRMS m/z (EI): calculated for $\mathrm{C}_{26} \mathrm{H}_{15} \mathrm{~F}_{3} \mathrm{~N}_{2}[\mathrm{M}]^{+}$412.1187, found 412.1185.

\section{1-Chloro-8-phenylphenanthro $\left[9^{\prime}, 10^{\prime}: 4,5\right]$ imidazo $[1,2-\alpha]$ pyridine (3am)}<smiles>Clc1ccn2c(c1)nc1c(-c3ccccc3)cccc12</smiles>

Following the general procedure, the reaction of $\mathbf{1 m}(45.7 \mathrm{mg}, 0.2 \mathrm{mmol})$, iodonium salts $\mathbf{2 a}(258 \mathrm{mg}$, $0.6 \mathrm{mmol}), \mathrm{Pd}(\mathrm{OAc})_{2}(4.5 \mathrm{mg}, 10 \mathrm{~mol} \%), \mathrm{K}_{2} \mathrm{HPO}_{4}(69.7 \mathrm{mg}, 0.4 \mathrm{mmol}$.) and acetic acid (4 mL) at 100 ${ }^{\circ} \mathrm{C}$ for $24 \mathrm{~h}$. The product was purified by flash chromatography (Petroleum ether/EtOAc, 5/1) to give 3am $53.8 \mathrm{mg}(71 \%)$ as a yellow solid: M. p.: 187-190 ${ }^{\circ} \mathrm{C} .{ }^{\mathbf{1}} \mathbf{H}$ NMR (400 $\left.\mathbf{M H z}, \mathbf{C D C l}_{\mathbf{3}}\right) \delta 8.84(\mathrm{~d}, J=$ $7.6 \mathrm{~Hz}, 2 \mathrm{H}), 8.74(\mathrm{~d}, J=7.7 \mathrm{~Hz}, 1 \mathrm{H}), 8.29$ (dd, $J=8.1,0.9 \mathrm{~Hz}, 1 \mathrm{H}), 7.74-7.69(\mathrm{~m}, 1 \mathrm{H}), 7.66$ (dd, $J=$ 8.1, 1.2 Hz, 1H), $7.61(\mathrm{dtd}, J=7.1,4.1,1.3 \mathrm{~Hz}, 2 \mathrm{H}), 7.57-7.53(\mathrm{~m}, 2 \mathrm{H}), 7.52-7.48(\mathrm{~m}, 4 \mathrm{H}), 6.83(\mathrm{dd}$, $J=7.5,2.2 \mathrm{~Hz}, 1 \mathrm{H}) .{ }^{13} \mathbf{C}$ NMR (101 MHz, $\left.\mathbf{C D C l}_{3}\right) \delta 145.75,143.95,141.74,140.59,131.49,131.01$, $130.88,129.98,128.68,127.51,127.49,126.74,126.24,126.06,125.05,125.04,124.70,123.22,122.75$, 120.16, 119.36, 117.60, 113.57, 77.48, 77.16, 76.84. HRMS m/z (EI): calculated for $\mathrm{C}_{25} \mathrm{H}_{15} \mathrm{ClN}_{2}[\mathrm{M}]^{+}$ 378.0924 , found 378.0915 . 


\section{6,11-Dimethyl-8-phenylphenanthro $\left[9^{\prime}, 10^{\prime}: 4,5\right]$ imidazo[1,2- $\left.\alpha\right]$ pyridine (3an)}

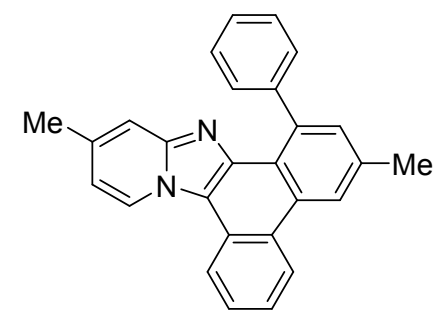

Following the general procedure, the reaction of $\mathbf{1 n}(44.4 \mathrm{mg}, 0.2 \mathrm{mmol})$, iodonium salts $2 \mathbf{a}(258 \mathrm{mg}$, $0.6 \mathrm{mmol}), \mathrm{Pd}(\mathrm{OAc})_{2}(4.5 \mathrm{mg}, 10 \mathrm{~mol} \%), \mathrm{K}_{2} \mathrm{HPO}_{4}(69.7 \mathrm{mg}, 0.4 \mathrm{mmol}$.) and acetic acid (4 mL) at 100 ${ }^{\circ} \mathrm{C}$ for $24 \mathrm{~h}$. The product was purified by flash chromatography (Petroleum ether/EtOAc, 5/1) to give 3an $60.3 \mathrm{mg}(81 \%)$ as a yellow solid: M. p.: $235-237^{\circ} \mathrm{C} . \mathbf{~}^{\mathbf{1}} \mathbf{H}$ NMR (400 $\left.\mathbf{M H z}, \mathbf{C D C l}_{3}\right) \delta 8.92(\mathrm{dd}, J=10.8$, $7.9 \mathrm{~Hz}, 2 \mathrm{H}), 8.60$ (s, 1H), 8.45 (d, $J=8.2 \mathrm{~Hz}, 1 \mathrm{H}), 7.77-7.70(\mathrm{~m}, 1 \mathrm{H}), 7.66-7.60$ (m, 1H), 7.57 (dd, $J$ $=7.5,2.0 \mathrm{~Hz}, 2 \mathrm{H}), 7.49(\mathrm{qd}, J=4.5,2.1 \mathrm{~Hz}, 3 \mathrm{H}), 7.45(\mathrm{~d}, J=1.3 \mathrm{~Hz}, 1 \mathrm{H}), 7.28(\mathrm{~d}, J=0.7 \mathrm{~Hz}, 1 \mathrm{H}), 6.75$ $(\mathrm{dd}, J=7.2,1.7 \mathrm{~Hz}, 1 \mathrm{H}), 2.67$ (s, 3H), 2.39 (s, 3H). ${ }^{13} \mathbf{C}$ NMR (101 MHz, $\left.\mathbf{C D C l}_{3}\right) \delta$ 146.66, 144.21, $141.13,140.32$, 136.38, 135.44, 132.59, 130.99, 130.14, 128.25, 127.51, 127.34, 126.62, 125.21, 125.04, $124.40,123.95,122.84,122.76,119.54,119.45,117.34,114.91,22.07,21.34$. HRMS m/z (EI): calculated for $\mathrm{C}_{27} \mathrm{H}_{20} \mathrm{~N}_{2}[\mathrm{M}]^{+} 372.1626$, found 372.1617 .

\section{6-Bromo-11-methyl-8-phenylphenanthro $\left[9^{\prime}, 10^{\prime}: 4,5\right]$ imidazo $[1,2-\alpha]$ pyridine (3ao)}<smiles></smiles>

Following the general procedure, the reaction of $\mathbf{1 0}(57.4 \mathrm{mg}, 0.2 \mathrm{mmol})$, iodonium salts $\mathbf{2 a}(258 \mathrm{mg}, 0.6$ $\mathrm{mmol}), \mathrm{Pd}(\mathrm{OAc})_{2}(4.5 \mathrm{mg}, 10 \mathrm{~mol} \%), \mathrm{K}_{2} \mathrm{HPO}_{4}\left(69.7 \mathrm{mg}, 0.4 \mathrm{mmol}\right.$.) and acetic acid $(4 \mathrm{~mL})$ at $100{ }^{\circ} \mathrm{C}$ for $24 \mathrm{~h}$. The product was purified by flash chromatography (Petroleum ether/EtOAc, 5/1) to give 3ao $49.8 \mathrm{mg}(57 \%)$ as a yellow solid: M. p.: $265-268{ }^{\circ} \mathrm{C} .{ }^{1} \mathbf{H}$ NMR (600 MHz, $\left.\mathbf{C D C l}_{3}\right) \delta 8.95(\mathrm{~d}, J=7.1 \mathrm{~Hz}$, $1 \mathrm{H}), 8.91(\mathrm{~d}, J=1.7 \mathrm{~Hz}, 1 \mathrm{H}), 8.82(\mathrm{~d}, J=8.3 \mathrm{~Hz}, 1 \mathrm{H}), 8.47(\mathrm{~d}, J=8.0 \mathrm{~Hz}, 1 \mathrm{H}), 7.80-7.76(\mathrm{~m}, 1 \mathrm{H})$, $7.71(\mathrm{~d}, J=1.9 \mathrm{~Hz}, 1 \mathrm{H}), 7.67-7.63(\mathrm{~m}, 1 \mathrm{H}), 7.54-7.50(\mathrm{~m}, 2 \mathrm{H}), 7.49-7.46(\mathrm{~m}, 3 \mathrm{H}), 7.27(\mathrm{~s}, 1 \mathrm{H})$, $6.80(\mathrm{dd}, J=7.1,1.6 \mathrm{~Hz}, 1 \mathrm{H}), 2.41$ (s, 3H). ${ }^{13} \mathbf{C}$ NMR (151 MHz, $\left.\mathbf{C D C l}_{3}\right) \delta 146.88,142.87,142.42$, $140.89,136.64,133.37,132.31,129.91,128.08,127.58,127.28,127.07,125.55,125.25,125.16,124.73$, 124.17, 124.00, 120.14, 120.02, 119.52, 117.57, 115.15, 21.37. HRMS m/z (EI): calculated for $\mathrm{C}_{26} \mathrm{H}_{17} \mathrm{BrN}_{2}[\mathrm{M}]^{+}$436.0575, found 436.0574. 


\section{1-Methoxy-6-methyl-8-phenylphenanthro $\left[9^{\prime}, 10^{\prime}: 4,5\right]$ imidazo $[1,2-\alpha]$ pyridine}

(3ap)

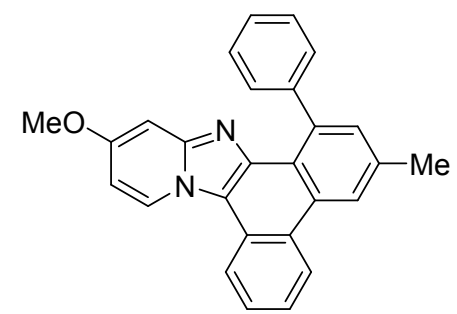

Following the general procedure, the reaction of $\mathbf{1 p}(47.6 \mathrm{mg}, 0.2 \mathrm{mmol})$, iodonium salts $2 \mathbf{a}(258 \mathrm{mg}$, $0.6 \mathrm{mmol}), \mathrm{Pd}(\mathrm{OAc})_{2}(4.5 \mathrm{mg}, 10 \mathrm{~mol} \%), \mathrm{K}_{2} \mathrm{HPO}_{4}(69.7 \mathrm{mg}, 0.4 \mathrm{mmol}$.) and acetic acid (4 mL) at 100 ${ }^{\circ} \mathrm{C}$ for $24 \mathrm{~h}$. The product was purified by flash chromatography (Petroleum ether/EtOAc, 5/1) to give 3ap $47.4 \mathrm{mg}(61 \%)$ as a yellow solid: M. p.: 239-241 ${ }^{\circ} \mathrm{C} .{ }^{1} \mathbf{H}$ NMR (400 MHz, $\left.\mathbf{C D C l}_{\mathbf{3}}\right) \delta 8.91(\mathrm{~d}, J=7.8 \mathrm{~Hz}$, $2 \mathrm{H}), 8.60(\mathrm{~s}, 1 \mathrm{H}), 8.41(\mathrm{~d}, J=7.6 \mathrm{~Hz}, 1 \mathrm{H}), 7.76-7.69(\mathrm{~m}, 1 \mathrm{H}), 7.64-7.56(\mathrm{~m}, 3 \mathrm{H}), 7.52-7.46(\mathrm{~m}$, $3 \mathrm{H}), 7.43(\mathrm{~d}, J=1.1 \mathrm{~Hz}, 1 \mathrm{H}), 6.78(\mathrm{~d}, J=2.6 \mathrm{~Hz}, 1 \mathrm{H}), 6.66(\mathrm{dd}, J=7.6,2.7 \mathrm{~Hz}, 1 \mathrm{H}), 3.84(\mathrm{~s}, 3 \mathrm{H}), 2.67$ (s, 3H). ${ }^{13}$ C NMR (101 MHz, $\left.\mathbf{C D C l}_{3}\right) \delta 158.15,148.05,144.22,141.07,140.14,135.29,132.63,130.83$, $130.13,128.00,127.55,127.35,126.63,126.53,125.06,124.25,123.68,122.81,122.48,119.36,119.04$, 107.39, 95.86, 55.75, 22.06. HRMS m/z (EI): calculated for $\mathrm{C}_{27} \mathrm{H}_{20} \mathrm{~N}_{2} \mathrm{O}[\mathrm{M}]^{+} 388.1576$, found 388.1573.

\section{1-Chloro-6-methyl-8-phenylphenanthro $\left[9^{\prime}, 10^{\prime}: 4,5\right]$ imidazo $[1,2-\alpha]$ pyridine (3aq)}<smiles></smiles>

Following the general procedure, the reaction of $\mathbf{1 q}(48.5 \mathrm{mg}, 0.2 \mathrm{mmol})$, iodonium salts $2 \mathbf{a}(258 \mathrm{mg}$, $0.6 \mathrm{mmol}), \mathrm{Pd}(\mathrm{OAc})_{2}(4.5 \mathrm{mg}, 10 \mathrm{~mol} \%), \mathrm{K}_{2} \mathrm{HPO}_{4}(69.7 \mathrm{mg}, 0.4 \mathrm{mmol}$.) and acetic acid (4 mL) at 100 ${ }^{\circ} \mathrm{C}$ for $24 \mathrm{~h}$. The product was purified by flash chromatography (Petroleum ether/EtOAc, 5/1) to give 3aq $45.6 \mathrm{mg}(58 \%)$ as a yellow solid: M. p.: 267-269 ${ }^{\circ}$ C. ${ }^{1} \mathbf{H}$ NMR (400 MHz, $\left.\mathbf{C D C l}_{3}\right) \delta 8.97-8.92(\mathrm{~m}$, $1 \mathrm{H}), 8.89(\mathrm{~d}, J=8.2 \mathrm{~Hz}, 1 \mathrm{H}), 8.57$ (s, $1 \mathrm{H}), 8.38$ (dd, $J=8.2,0.9 \mathrm{~Hz}, 1 \mathrm{H}), 7.75-7.69$ (m, 1H), 7.64 (ddd, $J=8.3,7.1,1.2 \mathrm{~Hz}, 1 \mathrm{H}), 7.57-7.50(\mathrm{~m}, 3 \mathrm{H}), 7.50-7.43(\mathrm{~m}, 4 \mathrm{H}), 6.90(\mathrm{dd}, J=7.5,2.2 \mathrm{~Hz}, 1 \mathrm{H}), 2.67$ (s, 3H). ${ }^{13} \mathbf{C}$ NMR (101 MHz, $\left.\mathbf{C D C l}_{3}\right) \delta 145.89,143.96,141.98,140.53,135.95,132.68,131.44,131.11$, $129.99,128.63,127.53,127.48,126.73,126.13,125.14,124.99,123.48,122.86,122.53,119.83,119.46$, 117.64, 113.57, 22.10. HRMS m/z (EI): calculated for $\mathrm{C}_{26} \mathrm{H}_{17} \mathrm{ClN}_{2}[\mathrm{M}]^{+}$392.1080, found 392.1075. 

midazo[1,2- $\alpha]$ pyridine (3ar)<smiles></smiles>

Following the general procedure, the reaction of $1 \mathbf{q}(48.5 \mathrm{mg}, 0.2 \mathrm{mmol})$, iodonium salts $2 \mathbf{e}$ ( $325 \mathrm{mg}, 0.6$ mmol), $\mathrm{Pd}(\mathrm{OAc})_{2}(4.5 \mathrm{mg}, 10 \mathrm{~mol} \%), \mathrm{K}_{2} \mathrm{HPO}_{4}\left(69.7 \mathrm{mg}, 0.4 \mathrm{mmol}\right.$ ) and acetic acid $(4 \mathrm{~mL})$ at $100{ }^{\circ} \mathrm{C}$ for $24 \mathrm{~h}$. The product was purified by flash chromatography (Petroleum ether/EtOAc, 5/1) to give 3ar $68.7 \mathrm{mg}(68 \%)$ as a yellow solid: M. p.: $292-294{ }^{\circ} \mathrm{C} .{ }^{1} \mathbf{H}$ NMR $\left(\mathbf{4 0 0} \mathbf{~ M H z}, \mathbf{C D C l}_{3}\right) \delta 8.98(\mathrm{~d}, J=7.5 \mathrm{~Hz}$, $1 \mathrm{H}), 8.84(\mathrm{~d}, J=8.9 \mathrm{~Hz}, 1 \mathrm{H}), 8.55(\mathrm{~s}, 1 \mathrm{H}), 8.41(\mathrm{~d}, J=1.8 \mathrm{~Hz}, 1 \mathrm{H}), 7.74(\mathrm{dd}, J=8.8,1.9 \mathrm{~Hz}, 1 \mathrm{H}), 7.48$ (dt, $J=5.5,4.6 \mathrm{~Hz}, 6 \mathrm{H}), 6.96$ (dd, $J=7.4,2.2 \mathrm{~Hz}, 1 \mathrm{H}), 2.66$ (s, 3H), 1.57 (s, 9H), 1.49 (s, 9H). ${ }^{13} \mathbf{C}$ NMR (101 MHz, $\mathbf{C D C l}_{3}$ ) $\delta$ 150.39, 149.57, 145.82, 142.15, 141.01, 140.48, 135.87, 132.42, 131.14, 129.60, $126.49,126.03,124.96,124.41,123.42,123.19,122.51,122.38,120.22,117.72,115.48,113.60,35.29$, 34.77, 31.77, 31.65, 22.08. HRMS m/z (EI): calculated for $\mathrm{C}_{34} \mathrm{H}_{33} \mathrm{ClN}_{2}[\mathrm{M}]^{+}$504.2332, found 504.2293.

\section{6-Chloro-2-methyl-8-(p-tolyl)phenanthro[9',10':4,5]imidazo[1,2- $\alpha]$ pyridine (3as)}

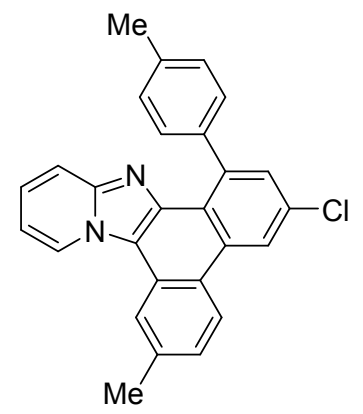

Following the general procedure, the reaction of $1 \mathbf{c}(45.7 \mathrm{mg}, 0.2 \mathrm{mmol})$, iodonium salts $2 \mathrm{~g}$ ( $275 \mathrm{mg}, 0.6$ mmol), $\mathrm{Pd}(\mathrm{OAc})_{2}(4.5 \mathrm{mg}, 10 \mathrm{~mol} \%), \mathrm{K}_{2} \mathrm{HPO}_{4}\left(69.7 \mathrm{mg}, 0.4 \mathrm{mmol}\right.$.) and acetic acid $(4 \mathrm{~mL})$ at $100{ }^{\circ} \mathrm{C}$ for $24 \mathrm{~h}$. The product was purified by flash chromatography (Petroleum ether/EtOAc, 5/1) to give 3as $55.3 \mathrm{mg}(68 \%)$ as a yellow solid: M. p.: $246-248{ }^{\circ} \mathrm{C} .{ }^{1} \mathbf{H}$ NMR $\left(\mathbf{4 0 0} \mathbf{M H z}, \mathbf{C D C l}_{3}\right) \delta 9.05(\mathrm{~d}, J=7.1 \mathrm{~Hz}$, $1 \mathrm{H}), 8.65(\mathrm{~d}, J=2.0 \mathrm{~Hz}, 1 \mathrm{H}), 8.63(\mathrm{~d}, J=8.6 \mathrm{~Hz}, 1 \mathrm{H}), 8.22(\mathrm{~s}, 1 \mathrm{H}), 7.54(\mathrm{t}, J=5.8 \mathrm{~Hz}, 2 \mathrm{H}), 7.46-7.40$ (m, 3H), $7.31-7.27$ (m, 2H), 7.25 (dd, $J=9.1,1.1 \mathrm{~Hz}, 1 \mathrm{H}), 6.96$ (td, $J=6.9,1.3 \mathrm{~Hz}, 1 \mathrm{H}), 2.65(\mathrm{~s}, 3 \mathrm{H})$, 2.52 (s, 3H). ${ }^{13} \mathbf{C}$ NMR (101 MHz, $\left.\mathbf{C D C l}_{\mathbf{3}}\right) \delta$ 146.18, 142.37, 140.95, 139.98, 138.03, 136.67, 132.29, $131.86,130.55,129.81,128.32,126.50,126.08,125.45,125.25,125.01,124.22,123.19,122.03,120.11$, $119.59,119.22,112.30,77.48,77.16,76.84,22.16,21.56$. HRMS m/z (EI): calculated for $\mathrm{C}_{27} \mathrm{H}_{19} \mathrm{ClN}_{2}$ $[\mathrm{M}]^{+} 406.1237$, found 406.1204 . 

a]pyridine (3at)

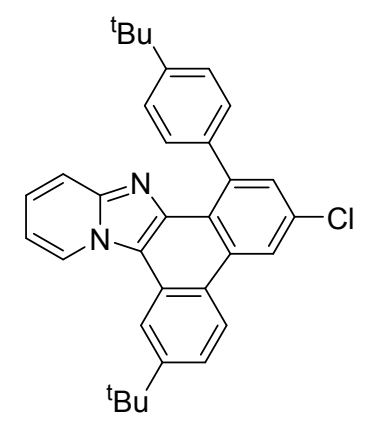

Following the general procedure, the reaction of $1 \mathbf{c}(45.7 \mathrm{mg}, 0.2 \mathrm{mmol})$, iodonium salts $2 \mathbf{e}$ ( $325 \mathrm{mg}, 0.6$ $\mathrm{mmol}), \mathrm{Pd}(\mathrm{OAc})_{2}(4.5 \mathrm{mg}, 10 \mathrm{~mol} \%), \mathrm{K}_{2} \mathrm{HPO}_{4}\left(69.7 \mathrm{mg}, 0.4 \mathrm{mmol}\right.$.) and acetic acid $(4 \mathrm{~mL})$ at $100{ }^{\circ} \mathrm{C}$ for $24 \mathrm{~h}$. The product was purified by flash chromatography (Petroleum ether/EtOAc, 5/1) to give 3at $58.9 \mathrm{mg}(60 \%)$ as a yellow solid: M. p.: $221-224{ }^{\circ} \mathrm{C} . \mathbf{~}^{1} \mathbf{H} \mathbf{N M R}\left(\mathbf{4 0 0} \mathbf{M H z}, \mathbf{C D C l}_{3}\right) \delta 9.10(\mathrm{~d}, J=6.7 \mathrm{~Hz}$, $1 \mathrm{H}), 8.74(\mathrm{~d}, J=8.9 \mathrm{~Hz}, 1 \mathrm{H}), 8.71(\mathrm{~d}, J=2.0 \mathrm{~Hz}, 1 \mathrm{H}), 8.51(\mathrm{~s}, 1 \mathrm{H}), 7.74(\mathrm{dd}, J=8.8,1.8 \mathrm{~Hz}, 1 \mathrm{H}), 7.59$ $(\mathrm{d}, J=2.0 \mathrm{~Hz}, 1 \mathrm{H}), 7.54-7.45(\mathrm{~m}, 5 \mathrm{H}), 7.29(\mathrm{dd}, J=9.0,6.7 \mathrm{~Hz}, 1 \mathrm{H}), 7.01(\mathrm{t}, J=6.8 \mathrm{~Hz}, 1 \mathrm{H}), 1.58(\mathrm{~d}$, $J=1.5 \mathrm{~Hz}, 9 \mathrm{H}), 1.48(\mathrm{~d}, J=3.1 \mathrm{~Hz}, 9 \mathrm{H}) .{ }^{13} \mathbf{C}$ NMR (101 MHz, $\left.\mathbf{C D C l}_{\mathbf{3}}\right) \delta 151.10,150.01,146.20,142.38$, $140.93,139.90,132.14,131.87,130.44,129.52,125.93,125.46,125.21,124.96,124.50,124.10,123.40$, 123.18, 122.06, 120.66, 119.33, 115.71, 112.48, 35.37, 34.79, 31.72, 31.64. HRMS m/z (EI): calculated for $\mathrm{C}_{33} \mathrm{H}_{31} \mathrm{ClN}_{2}[\mathrm{M}]^{+}$490.2176, found 490.2131 .

\section{2-Fluoro-8-(4-fluorophenyl)phenanthro $\left[9^{\prime}, 10^{\prime}: 4,5\right]$ imidazo[1,2- $\left.\alpha\right]$ pyridine (3ba)}

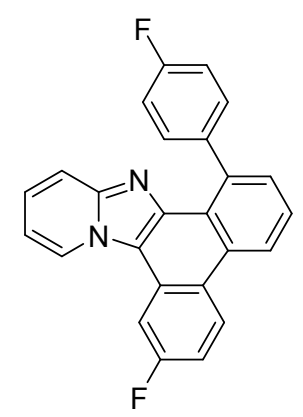

Following the general procedure, the reaction of $\mathbf{1 a}(38.8 \mathrm{mg}, 0.2 \mathrm{mmol})$, iodonium salts $\mathbf{2 b}(280 \mathrm{mg}$, $0.6 \mathrm{mmol}), \mathrm{Pd}(\mathrm{OAc})_{2}(4.5 \mathrm{mg}, 10 \mathrm{~mol} \%), \mathrm{K}_{2} \mathrm{HPO}_{4}(69.7 \mathrm{mg}, 0.4 \mathrm{mmol}$ ) and acetic acid $(4 \mathrm{~mL})$ at 100 ${ }^{\circ} \mathrm{C}$ for $24 \mathrm{~h}$. The product was purified by flash chromatography (Petroleum ether/DCM, 2/1) to give $3 \mathbf{b a}$ $52.5 \mathrm{mg}(69 \%)$ of $\mathbf{3 b a}$ as a white solid: M. p.: $253-255^{\circ} \mathrm{C} .{ }^{1} \mathbf{H}$ NMR (600 $\left.\mathbf{~ M H z}, \mathbf{C D C l}_{3}\right) \delta 9.00(\mathrm{~d}, J=$ $7.1 \mathrm{~Hz}, 1 \mathrm{H}), 8.89(\mathrm{dd}, J=9.2,5.8 \mathrm{~Hz}, 1 \mathrm{H}), 8.72(\mathrm{~d}, J=8.1 \mathrm{~Hz}, 1 \mathrm{H}), 8.14(\mathrm{dd}, J=10.5,2.5 \mathrm{~Hz}, 1 \mathrm{H}), 7.71$ (dd, $J=8.3,7.3 \mathrm{~Hz}, 1 \mathrm{H}), 7.58-7.55(\mathrm{~m}, 2 \mathrm{H}), 7.51-7.47$ (m, 2H), $7.40(\mathrm{ddd}, J=9.2,7.9,2.5 \mathrm{~Hz}, 1 \mathrm{H})$, 7.33 (ddd, $J=9.1,6.6,1.0 \mathrm{~Hz}, 1 \mathrm{H}), 7.18-7.12(\mathrm{~m}, 2 \mathrm{H}), 7.03(\mathrm{td}, J=6.9,1.2 \mathrm{~Hz}, 1 \mathrm{H}) .{ }^{13} \mathbf{C}$ NMR (151 MHz, $\left.\mathbf{C D C l}_{3}\right) \delta 162.39(\mathrm{~d}, J=244.6 \mathrm{~Hz}), 162.16(\mathrm{~d}, J=246.1 \mathrm{~Hz}), 146.50,142.03,139.85,139.83$, $139.75,131.58,131.52,130.75,130.69,127.44,127.38,126.36,125.80,125.76,124.61,122.82,119.31$, $114.31(\mathrm{~d}, J=22.6 \mathrm{~Hz}), 112.98$ (d, $J=22.6 \mathrm{~Hz}), 112.71,105.28(\mathrm{~d}, J=24.1 \mathrm{~Hz}) .{ }^{19} \mathbf{F}$ NMR (376 MHz, 
$\left.\mathbf{C D C l}_{3}\right) \delta-112.75(\mathrm{~s}),-116.97$ (s). HRMS m/z (EI): calculated for $\mathrm{C}_{25} \mathrm{H}_{14} \mathrm{~F}_{2} \mathrm{~N}_{2}[\mathrm{M}]^{+} 380.1125$, found 380.1122 .

\section{2-(Trifluoromethyl)-8-(4-(trifluoromethyl)phenyl)phenanthro $\left[9^{\prime}, 10^{\prime}: 4,5\right]$ imidazo[ 1,2- $\alpha$ ]pyridine (3ca)}<smiles></smiles>

Following the general procedure, the reaction of $1 \mathbf{a}(38.8 \mathrm{mg}, 0.2 \mathrm{mmol})$, iodonium salts $2 \mathrm{c}(340 \mathrm{mg}, 0.6$ $\mathrm{mmol}), \mathrm{Pd}(\mathrm{OAc})_{2}(4.5 \mathrm{mg}, 10 \mathrm{~mol} \%), \mathrm{K}_{2} \mathrm{HPO}_{4}\left(69.7 \mathrm{mg}, 0.4 \mathrm{mmol}\right.$.) and acetic acid $(4 \mathrm{~mL})$ at $100{ }^{\circ} \mathrm{C}$ for $24 \mathrm{~h}$. The product was purified by flash chromatography (Petroleum ether/DCM, 2/1) to give 3ca $54.8 \mathrm{mg}(57 \%)$ as a white solid: M. p.: $256-259{ }^{\circ} \mathrm{C} .{ }^{1} \mathbf{H}$ NMR (600 MHz, $\left.\mathbf{C D C l}_{3}\right) \delta 9.07(\mathrm{~d}, J=7.1 \mathrm{~Hz}$, $1 \mathrm{H}), 9.02(\mathrm{~d}, J=8.7 \mathrm{~Hz}, 1 \mathrm{H}), 8.85(\mathrm{~d}, J=8.1 \mathrm{~Hz}, 1 \mathrm{H}), 8.76(\mathrm{~s}, 1 \mathrm{H}), 7.89$ (dd, $J=8.7,1.2 \mathrm{~Hz}, 1 \mathrm{H}), 7.78$ $(\mathrm{dd}, J=8.3,7.3 \mathrm{~Hz}, 1 \mathrm{H}), 7.73(\mathrm{~d}, J=8.0 \mathrm{~Hz}, 2 \mathrm{H}), 7.63(\mathrm{dd}, J=10.6,4.4 \mathrm{~Hz}, 3 \mathrm{H}), 7.53(\mathrm{~d}, J=9.1 \mathrm{~Hz}$, 1H), $7.36(\mathrm{ddd}, J=9.1,6.6,1.0 \mathrm{~Hz}, 1 \mathrm{H}), 7.09(\mathrm{td}, J=6.9,1.2 \mathrm{~Hz}, 1 \mathrm{H}) .{ }^{13} \mathbf{C} \mathbf{N M R}\left(\mathbf{1 5 1} \mathbf{M H z}, \mathbf{C D C l}_{3}\right) \delta$ $147.25,146.52,141.56,139.26,131.53,130.51,130.21,129.97,126.41,126.04,125.79,125.73,125.44$, 124.74 (q, $J=271.8 \mathrm{~Hz}), 124.41$ (q, $J=271.8 \mathrm{~Hz}), 124.36$ (q, $J=4.5 \mathrm{~Hz}), 123.73,123.36,120.71(\mathrm{q}, J$ $=3.0 \mathrm{~Hz}), 119.66,119.27,116.43(\mathrm{q}, J=4.5 \mathrm{~Hz}), 113.01 .{ }^{19} \mathbf{F}$ NMR (376 MHz, $\left.\mathbf{C D C l}_{3}\right) \delta-61.88(\mathrm{dd}, J$ $=9.2,5.0 \mathrm{~Hz}),-62.03(\mathrm{dd}, J=9.3,4.8 \mathrm{~Hz}) . \quad$ HRMS m/z (EI): calculated for $\mathrm{C}_{27} \mathrm{H}_{14} \mathrm{~F}_{6} \mathrm{~N}_{2}[\mathrm{M}]^{+} 480.1061$, found 480.1052 .

\section{2-(tert-Butyl)-8-(4-(tert-Butyl)phenyl)phenanthro $\left[9^{\prime}, 10^{\prime}: 4,5\right]$ imidazo[1,2- $\left.\alpha\right]$ pyridi} ne (3da)

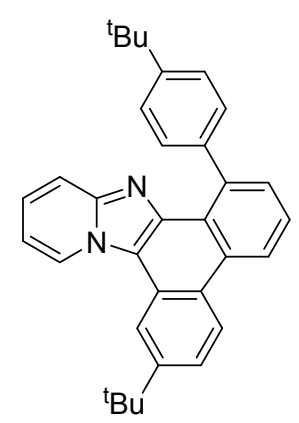

Following the general procedure, the reaction of $\mathbf{1 a}(38.8 \mathrm{mg}, 0.2 \mathrm{mmol})$, iodonium salts $\mathbf{2 d}$ (325 $\mathrm{mg}$, $0.6 \mathrm{mmol}), \mathrm{Pd}(\mathrm{OAc})_{2}(4.5 \mathrm{mg}, 10 \mathrm{~mol} \%), \mathrm{K}_{2} \mathrm{HPO}_{4}(69.7 \mathrm{mg}, 0.4 \mathrm{mmol}$ ) and acetic acid (4 mL) at 100 ${ }^{\circ} \mathrm{C}$ for $24 \mathrm{~h}$. The product was purified by flash chromatography (Petroleum ether/EtOAc, 5/1) to give 3da $66.7 \mathrm{mg}$ (73\%) as a yellow solid: M. p.: 264-266 ${ }^{\circ} \mathrm{C} .{ }^{1} \mathbf{H}$ NMR (400 MHz, $\left.\mathbf{C D C l}_{3}\right) \delta 9.11(\mathrm{~d}, J=7.1 \mathrm{~Hz}$, $1 \mathrm{H}), 8.86(\mathrm{~d}, J=8.9 \mathrm{~Hz}, 1 \mathrm{H}), 8.77(\mathrm{~d}, J=7.7 \mathrm{~Hz}, 1 \mathrm{H}), 8.52(\mathrm{~d}, J=1.8 \mathrm{~Hz}, 1 \mathrm{H}), 7.76(\mathrm{~d}, J=1.9 \mathrm{~Hz}, 1 \mathrm{H})$, $7.74(\mathrm{~d}, J=1.9 \mathrm{~Hz}, 1 \mathrm{H}), 7.73-7.67(\mathrm{~m}, 1 \mathrm{H}), 7.61(\mathrm{dd}, J=7.2,1.0 \mathrm{~Hz}, 1 \mathrm{H}), 7.52(\mathrm{~d}, J=11.2 \mathrm{~Hz}, 5 \mathrm{H})$, 
$7.31-7.27$ (m, 1H), $7.03-6.99$ (m, 1H), 1.57 (s, 9H), 1.48 (s, 9H). ${ }^{13} \mathbf{C}$ NMR (101 MHz, $\left.\mathbf{C D C l}_{\mathbf{3}}\right) \delta$ 150.41, 149.53, 146.07, 141.28, 141.21, 140.52, 130.90, 130.63, 129.68, 126.53, 126.03, 125.95, 125.06, 124.91, 124.88, 124.44, 123.76, 123.04, 122.47, 120.62, 119.29, 115.68, 112.38, 35.33, 34.77, 31.76, 31.69. HRMS m/z (EI): calculated for $\mathrm{C}_{33} \mathrm{H}_{32} \mathrm{~N}_{2}[\mathrm{M}]^{+} 456.2565$, found 456.2558 .

\section{2-Methoxy-8-(4-methoxyphenyl)phenanthro $\left[9^{\prime}, 10^{\prime}: 4,5\right]$ imidazo $[1,2-\alpha]$ pyridine} (3ea)<smiles></smiles>

Following the general procedure, the reaction of $1 \mathbf{a}(38.8 \mathrm{mg}, 0.2 \mathrm{mmol})$, iodonium salts $2 \mathbf{e}(294 \mathrm{mg}, 0.6$ $\mathrm{mmol}), \mathrm{Pd}(\mathrm{OAc})_{2}(4.5 \mathrm{mg}, 10 \mathrm{~mol} \%), \mathrm{K}_{2} \mathrm{HPO}_{4}\left(69.7 \mathrm{mg}, 0.4 \mathrm{mmol}\right.$.) and acetic acid $(4 \mathrm{~mL})$ at $100{ }^{\circ} \mathrm{C}$ for $24 \mathrm{~h}$. The product was purified by flash chromatography (Petroleum ether/EtOAc, 5/1) to give 3ea $58.2 \mathrm{mg}(72 \%)$ as a yellow solid: M. p.: $234-236^{\circ} \mathrm{C} .{ }^{1} \mathbf{H}$ NMR $\left(\mathbf{4 0 0} \mathbf{M H z}, \mathbf{C D C l}_{3}\right) \delta 9.01(\mathrm{~d}, J=7.1 \mathrm{~Hz}$, $1 \mathrm{H}), 8.79(\mathrm{~d}, J=9.3 \mathrm{~Hz}, 1 \mathrm{H}), 8.65(\mathrm{~d}, J=7.9 \mathrm{~Hz}, 1 \mathrm{H}), 7.87$ (d, $J=2.5 \mathrm{~Hz}, 1 \mathrm{H}), 7.67$ (dd, $J=8.2,7.4$ $\mathrm{Hz}, 1 \mathrm{H}), 7.58(\mathrm{~d}, J=9.1 \mathrm{~Hz}, 1 \mathrm{H}), 7.53(\mathrm{dd}, J=7.3,1.0 \mathrm{~Hz}, 1 \mathrm{H}), 7.52-7.49(\mathrm{~m}, 2 \mathrm{H}), 7.30-7.22(\mathrm{~m}$, 2H), $7.06-7.00$ (m, 2H), $6.97-6.92$ (m, 1H), 4.03 (s, 3H), 3.95 (s, 3H). ${ }^{13} \mathbf{C}$ NMR (101 MHz, $\left.\mathbf{C D C l}_{3}\right)$ $\delta 158.95,158.72,146.17,141.86,140.26,136.74,131.15,131.12,130.18,126.64,126.10,125.78$, $125.15,124.92,124.07,122.73,122.01,119.96,119.18,112.91,112.26,112.22,103.38,55.69,55.47$. HRMS m/z (EI): calculated for $\mathrm{C}_{27} \mathrm{H}_{20} \mathrm{~N}_{2} \mathrm{O}_{2}[\mathrm{M}]^{+} 404.1525$, found 404.1505 .

\section{2-Methyl-8-(p-tolyl)phenanthro[9',10':4,5]imidazo[1,2- $\alpha]$ pyridine (3fa)}

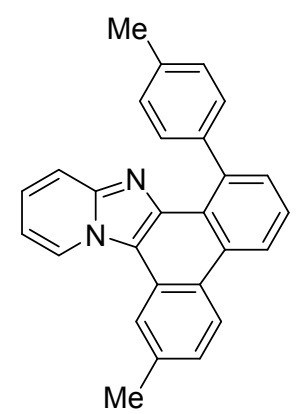

Following the general procedure, the reaction of $\mathbf{1 a}(38.8 \mathrm{mg}, 0.2 \mathrm{mmol})$, iodonium salts $\mathbf{2 f}(275 \mathrm{mg}, 0.6$ $\mathrm{mmol}), \mathrm{Pd}(\mathrm{OAc})_{2}(4.5 \mathrm{mg}, 10 \mathrm{~mol} \%), \mathrm{K}_{2} \mathrm{HPO}_{4}\left(69.7 \mathrm{mg}, 0.4 \mathrm{mmol}\right.$.) and acetic acid $(4 \mathrm{~mL})$ at $100{ }^{\circ} \mathrm{C}$ for $24 \mathrm{~h}$. The product was purified by flash chromatography (Petroleum ether/EtOAc, 5/1) to give $\mathbf{3 f a}$ $55.1 \mathrm{mg}(74 \%)$ as a yellow solid: M. p.: $230-232{ }^{\circ} \mathrm{C} .{ }^{1} \mathbf{H}$ NMR $\left(\mathbf{4 0 0} \mathbf{~ M H z}, \mathbf{C D C l}_{3}\right) \delta 9.12(\mathrm{dd}, J=7.1$, 
$1.0 \mathrm{~Hz}, 1 \mathrm{H}), 8.76(\mathrm{dd}, J=14.6,8.3 \mathrm{~Hz}, 2 \mathrm{H}), 8.29(\mathrm{~s}, 1 \mathrm{H}), 7.69(\mathrm{dd}, J=8.3,7.3 \mathrm{~Hz}, 1 \mathrm{H}), 7.63-7.53(\mathrm{~m}$, 2H), $7.48(\mathrm{~d}, J=7.9 \mathrm{~Hz}, 3 \mathrm{H}), 7.33-7.20(\mathrm{~m}, 3 \mathrm{H}), 6.97$ (t, $J=6.8 \mathrm{~Hz}, 1 \mathrm{H}), 2.68(\mathrm{~s}, 3 \mathrm{H}), 2.52(\mathrm{~s}, 3 \mathrm{H})$. ${ }^{13}$ C NMR (101 MHz, $\left.\mathbf{C D C l}_{3}\right) \delta 146.06,141.30,141.28,140.49,137.32,136.16,131.04,130.78,130.00$, $128.25,126.51,126.37,126.09,126.01,125.08,124.98,124.65,123.91,122.42,120.08,119.59,119.16$, 112.18, 77.48, 77.16, 76.84, 22.13, 21.54. HRMS m/z (EI): calculated for $\mathrm{C}_{27} \mathrm{H}_{20} \mathrm{~N}_{2}[\mathrm{M}]^{+} 372.1626$, found 372.1622 .

\section{8-([1,1'-Biphenyl]-4-yl)-2-phenylphenanthro[9',10':4,5]imidazo[1,2- $\alpha]$ pyridine} (3ga)

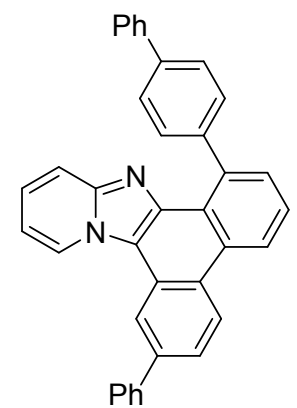

Following the general procedure, the reaction of $1 \mathrm{a}(38.8 \mathrm{mg}, 0.2 \mathrm{mmol})$, iodonium salts $\mathbf{2 g}$ ( $349 \mathrm{mg}, 0.6$ $\mathrm{mmol}), \mathrm{Pd}(\mathrm{OAc})_{2}(4.5 \mathrm{mg}, 10 \mathrm{~mol} \%), \mathrm{K}_{2} \mathrm{HPO}_{4}\left(69.7 \mathrm{mg}, 0.4 \mathrm{mmol}\right.$.) and acetic acid $(4 \mathrm{~mL})$ at $100{ }^{\circ} \mathrm{C}$ for $24 \mathrm{~h}$. The product was purified by flash chromatography (Petroleum ether/EtOAc, 5/1) to give 3ga $77.5 \mathrm{mg}(78 \%)$ as a yellow solid: M. p.: 241-244 ${ }^{\circ} \mathrm{C} .{ }^{1} \mathbf{H}$ NMR (600 MHz, $\left.\mathbf{C D C l}_{3}\right) \delta 9.17(\mathrm{~d}, J=7.1 \mathrm{~Hz}$, $1 \mathrm{H}), 8.97$ (d, $J=8.7 \mathrm{~Hz}, 1 \mathrm{H}), 8.83(\mathrm{~d}, J=8.0 \mathrm{~Hz}, 1 \mathrm{H}), 8.69$ (d, $J=1.6 \mathrm{~Hz}, 1 \mathrm{H}), 7.90$ (dd, $J=8.6,1.7$ $\mathrm{Hz}, 1 \mathrm{H}), 7.86-7.82(\mathrm{~m}, 2 \mathrm{H}), 7.82-7.79(\mathrm{~m}, 2 \mathrm{H}), 7.77-7.73(\mathrm{~m}, 3 \mathrm{H}), 7.69-7.65(\mathrm{~m}, 3 \mathrm{H}), 7.60-7.46$ $(\mathrm{m}, 6 \mathrm{H}), 7.40(\mathrm{t}, J=7.4 \mathrm{~Hz}, 1 \mathrm{H}), 7.30-7.27(\mathrm{~m}, 1 \mathrm{H}), 6.99(\mathrm{td}, J=6.9,1.1 \mathrm{~Hz}, 1 \mathrm{H}) .{ }^{13} \mathbf{C}$ NMR (101 MHz, $\left.\mathbf{C D C l}_{3}\right) \delta 146.29,143.18,141.63,141.54,141.22,140.42,140.24,139.45,131.05,130.87,130.58$, 129.24, 128.91, 127.94, 127.77, 127.74, 127.31, 127.17, 126.28, 126.24, 126.07, 125.63, 125.36, 125.04, 124.29, 124.16, 122.94, 120.33, 119.32, 118.09, 112.52. HRMS m/z (EI): calculated for $\mathrm{C}_{37} \mathrm{H}_{24} \mathrm{~N}_{2}[\mathrm{M}]^{+}$ 496.1939, found 496.1938 .

\section{4-Fluoro-8-(2-fluorophenyl)phenanthro[9',10':4,5]imidazo[1,2-a]pyridine (3ha)}

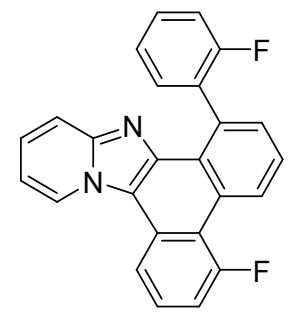

Following the general procedure, the reaction of $\mathbf{1 a}(38.8 \mathrm{mg}, 0.2 \mathrm{mmol})$, iodonium salts $\mathbf{2 h}(280 \mathrm{mg}$, $0.6 \mathrm{mmol}), \mathrm{Pd}(\mathrm{OAc})_{2}(4.5 \mathrm{mg}, 10 \mathrm{~mol} \%), \mathrm{K}_{2} \mathrm{HPO}_{4}(69.7 \mathrm{mg}, 0.4 \mathrm{mmol}$.) and acetic acid (4 mL) at 100 ${ }^{\circ} \mathrm{C}$ for $24 \mathrm{~h}$. The product was purified by flash chromatography (DCM) to give $\mathbf{3 h a} 54.8 \mathrm{mg}(72 \%)$ as a yellow solid: M. p.: $229-231{ }^{\circ} \mathrm{C} .{ }^{1} \mathbf{H}$ NMR (600 $\left.\mathbf{M H z}, \mathbf{C D C l}_{3}\right) \delta 9.30(\mathrm{dd}, J=8.3,4.5 \mathrm{~Hz}, 1 \mathrm{H}), 9.00(\mathrm{~d}$, $J=7.0 \mathrm{~Hz}, 1 \mathrm{H}), 8.25$ (d, $J=8.0 \mathrm{~Hz}, 1 \mathrm{H}), 7.76(\mathrm{t}, J=7.7 \mathrm{~Hz}, 1 \mathrm{H}), 7.65$ (dd, $J=12.4,7.6 \mathrm{~Hz}, 2 \mathrm{H}), 7.53$ 
(t, $J=6.9 \mathrm{~Hz}, 1 \mathrm{H}), 7.48(\mathrm{dd}, J=11.1,5.8 \mathrm{~Hz}, 2 \mathrm{H}), 7.34(\mathrm{dt}, J=23.4,7.6 \mathrm{~Hz}, 2 \mathrm{H}), 7.29-7.23(\mathrm{~m}, 1 \mathrm{H})$, $7.15(\mathrm{t}, J=9.0 \mathrm{~Hz}, 1 \mathrm{H}), 6.93(\mathrm{t}, J=6.7 \mathrm{~Hz}, 1 \mathrm{H}) .{ }^{13} \mathbf{C}$ NMR (151 MHz, CDCl$) \delta 162.26(\mathrm{~d}, J=253.7$ $\mathrm{Hz}), 161.35$ (d, $J=253.7 \mathrm{~Hz}), 146.94,141.97,133.38,132.38$ (d, $J=16.6 \mathrm{~Hz}), 131.13$ (d, $J=3.0 \mathrm{~Hz}$ ), 128.72 (d, $J=7.6 \mathrm{~Hz}), 128.72,128.47,128.28,127.95$ (d, $J=10.6 \mathrm{~Hz}), 126.44$ (d, $J=1.5 \mathrm{~Hz}), 126.27$ (d, $J=13.6 \mathrm{~Hz}), 126.29,126.06,125.54,123.74$ (d, $J=3.0 \mathrm{~Hz}), 119.79$ (d, $J=4.5 \mathrm{~Hz}), 119.25,117.56$ $(\mathrm{d}, J=7.6 \mathrm{~Hz}), 115.35(\mathrm{~d}, J=3.0 \mathrm{~Hz}), 114.68(\mathrm{~d}, J=22.6 \mathrm{~Hz}), 112.66,112.49 .{ }^{19} \mathbf{F} \mathbf{~ N M R}(\mathbf{5 6 5} \mathbf{~ M H z}$, $\left.\mathbf{C D C l}_{3}\right) \delta-104.73--104.86(\mathrm{~m}),-114.28(\mathrm{dd}, J=13.3,8.5 \mathrm{~Hz})$. HRMS m/z (EI): calculated for $\mathrm{C}_{25} \mathrm{H}_{14} \mathrm{~F}_{2} \mathrm{~N}_{2}[\mathrm{M}]^{+}$380.1125, found 380.1122 .

\section{2-(Trifluoromethoxy)-8-(4-(trifluoromethoxy)phenyl)phenanthro $\left[9^{\prime}, 10^{\prime}: 4,5\right]$ imid azo[1,2- $\alpha]$ pyridine (3ia)}

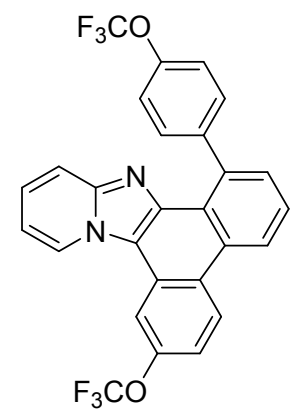

Following the general procedure, the reaction of $\mathbf{1 a}(38.8 \mathrm{mg}, 0.2 \mathrm{mmol})$, iodonium salts $\mathbf{2 i}$ ( $359 \mathrm{mg}, 0.6$ $\mathrm{mmol}), \mathrm{Pd}(\mathrm{OAc})_{2}(4.5 \mathrm{mg}, 10 \mathrm{~mol} \%), \mathrm{K}_{2} \mathrm{HPO}_{4}\left(69.7 \mathrm{mg}, 0.4 \mathrm{mmol}\right.$.) and acetic acid $(4 \mathrm{~mL})$ at $100{ }^{\circ} \mathrm{C}$ for $24 \mathrm{~h}$. The product was purified by flash chromatography (Petroleum ether/EtOAc, 5/1) to give 3ia $62.5 \mathrm{mg}(63 \%)$ as a white solid: M. p.: $\left.192-195{ }^{\circ} \mathrm{C} .{ }^{1} \mathbf{H} \mathbf{~ N M R ~ ( 6 0 0 ~} \mathbf{M H z}, \mathbf{C D C l}_{3}\right) \delta 8.95(\mathrm{~d}, J=7.1 \mathrm{~Hz}$, $1 \mathrm{H}), 8.88(\mathrm{~d}, J=9.2 \mathrm{~Hz}, 1 \mathrm{H}), 8.71(\mathrm{~d}, J=8.1 \mathrm{~Hz}, 1 \mathrm{H}), 8.26(\mathrm{~d}, J=1.8 \mathrm{~Hz}, 1 \mathrm{H}), 7.71(\mathrm{dd}, J=8.2,7.3$ $\mathrm{Hz}, 1 \mathrm{H}), 7.58(\mathrm{dd}, J=7.2,0.9 \mathrm{~Hz}, 1 \mathrm{H}), 7.54-7.49(\mathrm{~m}, 4 \mathrm{H}), 7.35-7.31(\mathrm{~m}, 3 \mathrm{H}), 7.04(\mathrm{td}, J=6.9,1.2$ Hz, 1H). ${ }^{13} \mathbf{C}$ NMR (151 MHz, $\left.\mathbf{C D C l}_{3}\right) \delta 148.50,148.46,146.56,142.52,141.91,139.38,131.35,130.92$, $130.26,126.96,126.83,126.42,126.00,125.69,124.94,124.57,123.18,120.91$ (q, $J=256.7 \mathrm{~Hz}$ ), 120.85 (q, $J=258.2 \mathrm{~Hz}$ ), 120.08, 119.52, 119.27, 117.28, 112.92, 111.32. ${ }^{19} \mathbf{F}$ NMR (376 $\left.\mathbf{~ M H z}, \mathbf{C D C l}_{\mathbf{3}}\right)$ $\delta$-57.34 (s), -57.63 (s). HRMS m/z (EI): calculated for $\mathrm{C}_{27} \mathrm{H}_{14} \mathrm{~F}_{6} \mathrm{~N}_{2} \mathrm{O}_{2}[\mathrm{M}]^{+} 512.0959$, found 512.0953 .

\section{2-Phenoxy-8-(4-phenoxyphenyl)phenanthro $\left[9^{\prime}, 10^{\prime}: 4,5\right]$ imidazo $[1,2-\alpha]$ pyridine} (3ja)

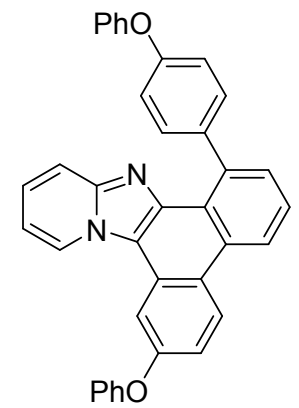

Following the general procedure, the reaction of $\mathbf{1 a}(38.8 \mathrm{mg}, 0.2 \mathrm{mmol})$, iodonium salts $\mathbf{2 j}$ ( $369 \mathrm{mg}, 0.6$ mmol), $\mathrm{Pd}(\mathrm{OAc})_{2}(4.5 \mathrm{mg}, 10 \mathrm{~mol} \%), \mathrm{K}_{2} \mathrm{HPO}_{4}\left(69.7 \mathrm{mg}, 0.4 \mathrm{mmol}\right.$.) and acetic acid $(4 \mathrm{~mL})$ at $100{ }^{\circ} \mathrm{C}$ 
for $24 \mathrm{~h}$. The product was purified by flash chromatography (Petroleum ether/EtOAc, 5/1) to give $\mathbf{3 j a}$ $50.7 \mathrm{mg}$ (48\%) as a yellow solid: M. p.: $200-202{ }^{\circ} \mathrm{C} .{ }^{1} \mathbf{H}$ NMR (400 MHz, $\left.\mathbf{C D C l}_{3}\right) \delta 8.89$ (d, $J=7.1 \mathrm{~Hz}$, $1 \mathrm{H}), 8.86(\mathrm{~d}, J=9.3 \mathrm{~Hz}, 1 \mathrm{H}), 8.71(\mathrm{~d}, J=7.9 \mathrm{~Hz}, 1 \mathrm{H}), 8.12(\mathrm{~d}, J=2.4 \mathrm{~Hz}, 1 \mathrm{H}), 7.74-7.69(\mathrm{~m}, 1 \mathrm{H})$, $7.60(\mathrm{dd}, J=12.8,5.5 \mathrm{~Hz}, 2 \mathrm{H}), 7.56-7.51(\mathrm{~m}, 2 \mathrm{H}), 7.47-7.38(\mathrm{~m}, 4 \mathrm{H}), 7.35-7.28(\mathrm{~m}, 2 \mathrm{H}), 7.21(\mathrm{dd}$, $J=9.7,4.4 \mathrm{~Hz}, 4 \mathrm{H}), 7.19-7.10$ (m, 4H), 6.93 (td, $J=6.9,1.2 \mathrm{~Hz}, 1 \mathrm{H}) .{ }^{13} \mathbf{C} \mathbf{~ N M R}\left(\mathbf{1 0 1} \mathbf{~ M H z}, \mathbf{C D C l}_{3}\right)$ $\delta 158.17,157.19,156.71,155.94,146.34,140.05,139.45,131.47,130.92,130.42,130.16,129.81$, 126.96, 126.28, 125.88, 125.62, 125.00, 124.48, 123.92, 122.92, 122.57, 119.93, 119.24, 119.08, 118.62, 118.53, 116.29, 112.49, 108.82. HRMS m/z (EI): calculated for $\mathrm{C}_{37} \mathrm{H}_{24} \mathrm{~N}_{2} \mathrm{O}_{2}[\mathrm{M}]^{+} 528.1838$, found 528.1833 .

\section{8-(3,4-Dimethylphenyl)-2,3-dimethylphenanthro $\left[9^{\prime}, 10^{\prime}: 4,5\right]$ imidazo[1,2- $\left.\alpha\right]$ pyridin -e (3ka)}

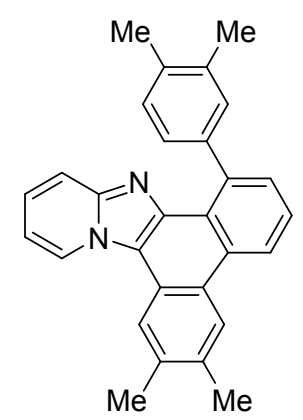

Following the general procedure, the reaction of $1 \mathrm{a}(38.8 \mathrm{mg}, 0.2 \mathrm{mmol})$, iodonium salts $2 \mathbf{k}(292 \mathrm{mg}$, $0.6 \mathrm{mmol}), \mathrm{Pd}(\mathrm{OAc})_{2}(4.5 \mathrm{mg}, 10 \mathrm{~mol} \%), \mathrm{K}_{2} \mathrm{HPO}_{4}(69.7 \mathrm{mg}, 0.4 \mathrm{mmol}$ ) and acetic acid $(4 \mathrm{~mL})$ at 100 ${ }^{\circ} \mathrm{C}$ for $24 \mathrm{~h}$. The product was purified by flash chromatography (Petroleum ether/EtOAc, 5/1) to give $3 \mathbf{k a}$ $34.4 \mathrm{mg}(43 \%)$ as a yellow solid: M. p.: $262-264{ }^{\circ} \mathrm{C} .{ }^{1} \mathbf{H}$ NMR (600 MHz, $\left.\mathbf{C D C l}_{3}\right) \delta 9.10(\mathrm{~d}, J=7.1 \mathrm{~Hz}$, $1 \mathrm{H}), 8.75(\mathrm{~d}, J=8.1 \mathrm{~Hz}, 1 \mathrm{H}), 8.64(\mathrm{~s}, 1 \mathrm{H}), 8.25(\mathrm{~s}, 1 \mathrm{H}), 7.67(\mathrm{dd}, J=13.6,5.5 \mathrm{~Hz}, 1 \mathrm{H}), 7.59-7.55(\mathrm{~m}$, 2H), $7.39(\mathrm{~s}, 1 \mathrm{H}), 7.32(\mathrm{dd}, J=7.6,1.4 \mathrm{~Hz}, 1 \mathrm{H}), 7.26-7.21(\mathrm{~m}, 2 \mathrm{H}), 6.95(\mathrm{td}, J=6.9,1.1 \mathrm{~Hz}, 1 \mathrm{H}), 2.58$ (s, 3H), 2.58 (s, 3H), 2.42 (s, 3H), 2.36 (s, 3H). ${ }^{13} \mathbf{C}$ NMR (151 MHz, $\mathbf{C D C l}_{3}$ ) $\delta$ 145.81, 141.64, 140.86, 140.61, 136.61, 135.37, 134.72, 133.74, 131.26, 130.82, 130.75, 128.69, 127.82, 127.15, 126.03, 125.84, 125.65, 124.81, 124.68, 122.33, 122.15, 120.21, 120.12, 119.21, 111.97, 20.69, 20.63, 20.14, 19.83. HRMS m/z (EI): calculated for $\mathrm{C}_{29} \mathrm{H}_{24} \mathrm{~N}_{2}[\mathrm{M}]^{+} 400.1939$, found 400.1937 . 


\section{Part 6. Mechanistic Studies}

(a)

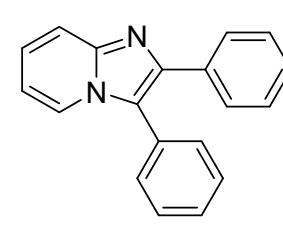

4

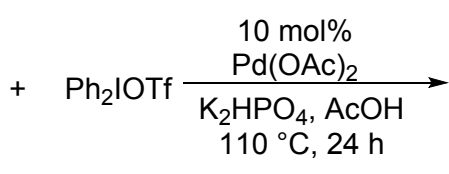

$2 a$

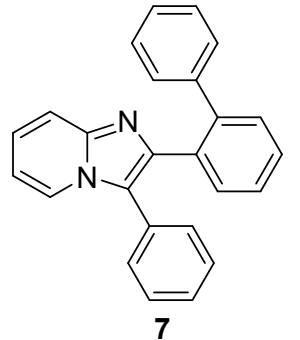

$89 \%$ yield

To an oven-dried Schlenk tube was added compound 4 (1.0 equiv.) in the presence of 3.0 equivalent of diaryliodonium salt 2a, 2.0 equivalent of $\mathrm{K}_{2} \mathrm{HPO}_{4}$ and $10 \mathrm{~mol} \%$ of $\mathrm{Pd}(\mathrm{OAc})_{2}$. The mixture was stirred at $100{ }^{\circ} \mathrm{C}$ in an oil bath. After 24 hours, the reaction mixture was filtered and the residue was washed with Sodium bicarbonate solution $(2 \times 50 \mathrm{~mL})$, then dried under vacuum and after column chromatography on silica gel afforded $61.7 \mathrm{mg}(89 \%)$ of 7 .

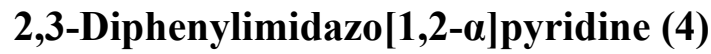

${ }^{1}$ H NMR (400 MHz, CDCl $\left.{ }_{3}\right) \delta 7.96(\mathrm{dt}, J=6.9,1.1 \mathrm{~Hz}, 1 \mathrm{H}), 7.68(\mathrm{ddt}, J=8.4,3.9,1.7 \mathrm{~Hz}, 3 \mathrm{H}), 7.56-$ $7.44(\mathrm{~m}, 5 \mathrm{H}), 7.32-7.24(\mathrm{~m}, 3 \mathrm{H}), 7.20(\mathrm{ddd}, J=9.0,6.7,1.2 \mathrm{~Hz}, 1 \mathrm{H}), 6.73(\mathrm{td}, J=6.8,1.2 \mathrm{~Hz}, 1 \mathrm{H})$.

${ }^{13}$ C NMR (101 MHz, $\mathbf{C D C l}_{3}$ ) $\delta$ 144.92, 142.53, 134.29, 130.82, 129.99, 129.64, 128.97, 128.36, 128.19, $127.56,124.74,123.37,121.18,117.65,112.35$.

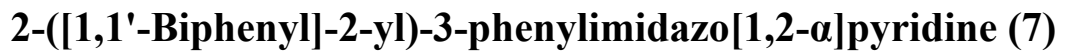

${ }^{1} \mathbf{H}$ NMR (400 MHz, $\left.\mathbf{C D C l}_{3}\right) \delta 8.00(\mathrm{dt}, J=6.9,1.0 \mathrm{~Hz}, 1 \mathrm{H}), 7.80(\mathrm{dd}, J=7.6,1.3 \mathrm{~Hz}, 1 \mathrm{H}), 7.72-7.67$ (m, 1H), $7.47-7.37(\mathrm{~m}, 2 \mathrm{H}), 7.22-7.09(\mathrm{~m}, 5 \mathrm{H}), 7.04-6.96(\mathrm{~m}, 1 \mathrm{H}), 6.95-6.88(\mathrm{~m}, 2 \mathrm{H}), 6.67$ (ddt, $J=5.5,3.4,1.4 \mathrm{~Hz}, 5 \mathrm{H}) .{ }^{13} \mathbf{C}$ NMR (101 MHz, $\left.\mathbf{C D C l}_{3}\right) \delta 144.93,143.47,141.67,141.47,133.04,131.95$, $130.15,129.05,128.90,128.73,128.28,127.65,127.43,127.38,125.76,124.26,123.10,122.45,117.88$, 112.24 .

(b)

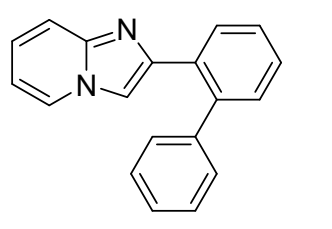

5

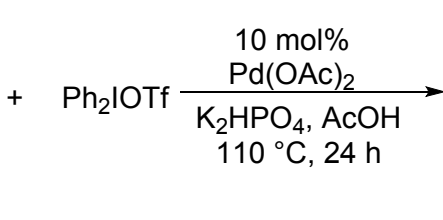

$2 a$

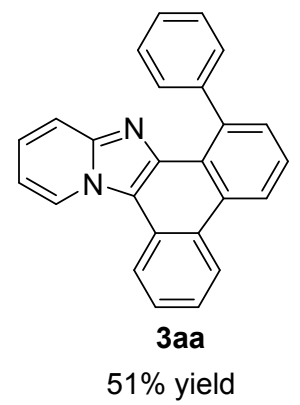

To an oven-dried Schlenk tube was added compound 5 (1.0 equiv.) in the presence of 3.0 equivalent of diaryliodonium salt 2a, 2.0 equivalent of $\mathrm{K}_{2} \mathrm{HPO}_{4}$ and $10 \mathrm{~mol} \%$ of $\mathrm{Pd}(\mathrm{OAc})_{2}$. The mixture was stirred at $100{ }^{\circ} \mathrm{C}$ in an oil bath. After 24 hours, the reaction mixture was filtered and the residue was washed with sodium bicarbonate solution $(2 \times 50 \mathrm{~mL})$, then dried under vacuum and after column chromatography on silica gel afforded $35.1 \mathrm{mg}(51 \%)$ of $\mathbf{3 a a}$. 


\section{2-([1,1'-Biphenyl]-2-yl)imidazo[1,2- $\alpha]$ pyridine $(5)^{\mathbf{7}}$}

${ }^{1} \mathbf{H}$ NMR (400 MHz, CDCl $) \delta 8.26(\mathrm{~d}, J=7.8 \mathrm{~Hz}, 1 \mathrm{H}), 7.76(\mathrm{dd}, J=6.8,1.0 \mathrm{~Hz}, 1 \mathrm{H}), 7.60(\mathrm{~d}, J=9.1$ $\mathrm{Hz}, 1 \mathrm{H}$ ), 7.50 (ddd, $J=8.7,7.6,1.4 \mathrm{~Hz}, 1 \mathrm{H}$ ), 7.37 (tdd, $J=10.1,7.1,2.0 \mathrm{~Hz}, 7 \mathrm{H}$ ), 7.09 (ddd, $J=9.0$, 6.7, $1.1 \mathrm{~Hz}, 1 \mathrm{H}), 6.64(\mathrm{t}, J=6.8 \mathrm{~Hz}, 1 \mathrm{H}), 6.55(\mathrm{~s}, 1 \mathrm{H}) .{ }^{13} \mathbf{C} \mathbf{~ N M R}\left(\mathbf{1 0 1} \mathbf{~ M H z}, \mathbf{C D C l}_{3}\right) \delta 144.46,144.33$, $142.47,140.62,132.38,130.45,129.64,129.55,128.38,127.82,127.62,127.19,125.54,124.25,117.44$, $112.08,111.42$.

(c)

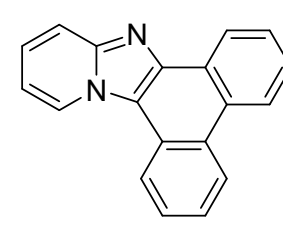

6

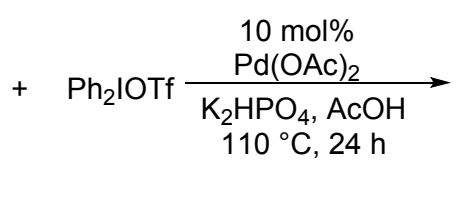

$2 \mathbf{a}$

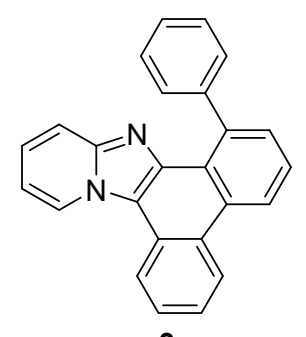

3aa

$16 \%$ yield

To an oven-dried Schlenk tube was added compound 6 (1.0 equiv.) in the presence of 3.0 equivalent of diaryliodonium salt 2a, 2.0 equivalent of $\mathrm{K}_{2} \mathrm{HPO}_{4}$ and $10 \mathrm{~mol} \%$ of $\mathrm{Pd}(\mathrm{OAc})_{2}$. The mixture was stirred at $100{ }^{\circ} \mathrm{C}$ in an oil bath. After 24 hours, the reaction mixture was filtered and the residue was washed with Sodium bicarbonate solution $(2 \times 50 \mathrm{~mL})$, then dried under vacuum and after column chromatography on silica gel afforded $11 \mathrm{mg}(16 \%)$ of 3aa.

\section{Phenanthro[ $\left[9^{\prime}, 10^{\prime}: 4,5\right]$ imidazo[1,2- $\left.\alpha\right]$ pyridine $(6)^{7}$}

${ }^{1}$ H NMR (400 MHz, CDCl $) \delta 9.07(\mathrm{~d}, J=7.1 \mathrm{~Hz}, 1 \mathrm{H}), 8.89(\mathrm{dd}, J=7.8,1.5 \mathrm{~Hz}, 1 \mathrm{H}), 8.81(\mathrm{~d}, J=8.2$ $\mathrm{Hz}, 1 \mathrm{H}), 8.69(\mathrm{~d}, J=8.0 \mathrm{~Hz}, 1 \mathrm{H}), 8.42(\mathrm{~d}, J=8.1 \mathrm{~Hz}, 1 \mathrm{H}), 7.94(\mathrm{~d}, J=9.1 \mathrm{~Hz}, 1 \mathrm{H}), 7.76(\mathrm{td}, J=7.6,1.2$ $\mathrm{Hz}, 1 \mathrm{H}), 7.71(\mathrm{dd}, J=12.0,4.4 \mathrm{~Hz}, 2 \mathrm{H}), 7.64-7.58(\mathrm{~m}, 1 \mathrm{H}), 7.43$ (ddd, $J=9.0,6.7,0.9 \mathrm{~Hz}, 1 \mathrm{H}), 7.04$ $(\operatorname{td}, J=6.9,1.1 \mathrm{~Hz}, 1 \mathrm{H}) .{ }^{13} \mathbf{C}$ NMR (101 MHz, $\left.\mathbf{C D C l}_{3}\right) \delta 147.58,141.08,129.73,128.38,127.49,127.40$, 127.14, 127.06, 126.60, 126.24, 124.81, 124.63, 123.90, 123.70, 123.31, 119.81, 119.31, 118.32, 112.56.

(d)

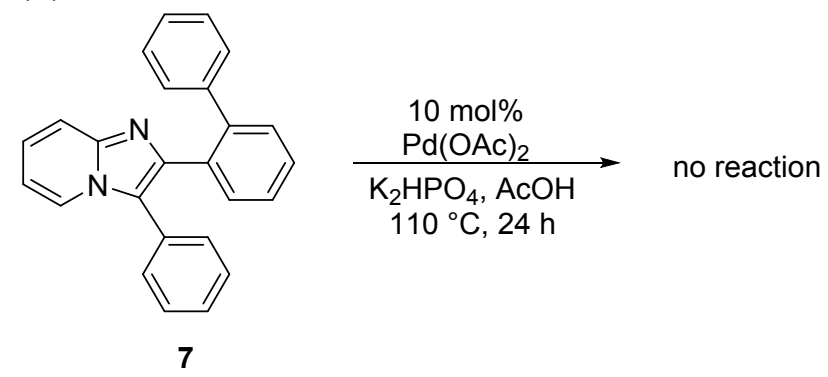

To an oven-dried Schlenk tube was added compound 7 (1.0 equiv.) in the presence of 2.0 equivalent of $\mathrm{K}_{2} \mathrm{HPO}_{4}$ and $10 \mathrm{~mol} \%$ of $\mathrm{Pd}(\mathrm{OAc})_{2}$. The mixture was stirred at $100{ }^{\circ} \mathrm{C}$ in an oil bath. After 24 hours, the desired compound 3aa was not found. 
(e)

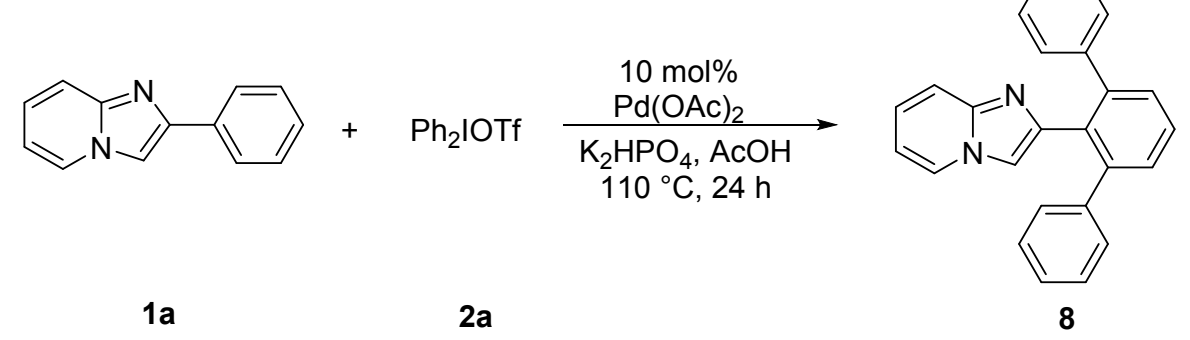

A scale-up reaction (10 gram) of modle reaction of $1 \mathbf{a}$ with $\mathbf{2 a}$ under the standard reaction, a seperable 8 was isolated and characterized by NMR and MS spectra.

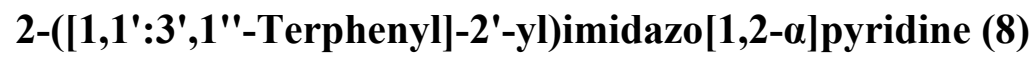

${ }^{1}$ H NMR (400 MHz, $\left.\mathbf{C D C l}_{3}\right) \delta 7.76(\mathrm{~d}, J=6.8 \mathrm{~Hz}, 1 \mathrm{H}), 7.51(\mathrm{dd}, J=8.6,6.5 \mathrm{~Hz}, 1 \mathrm{H}), 7.47-7.41(\mathrm{~m}$, 2H), $7.36(\mathrm{~d}, J=8.9 \mathrm{~Hz}, 1 \mathrm{H}), 7.23(\mathrm{dd}, J=7.6,1.9 \mathrm{~Hz}, 4 \mathrm{H}), 7.18-7.09(\mathrm{~m}, 6 \mathrm{H}), 7.02-6.94(\mathrm{~m}, 1 \mathrm{H})$, 6.92 (s, 1H), 6.58 (td, $J=6.7,0.8 \mathrm{~Hz}, 1 \mathrm{H}) .{ }^{13} \mathbf{C}$ NMR (101 MHz, $\mathbf{C D C l}_{3}$ ) $\delta$ 144.00, 143.28, 142.15, $131.82,129.55,129.41,128.24,127.61,126.35,125.33,123.60,117.52,112.54,111.77$. HRMS m/z (EI): calculated for $\mathrm{C}_{15} \mathrm{H}_{28} \mathrm{~N}_{2}[\mathrm{M}]^{+} 346.1470$, found 346.1440 .

(f)

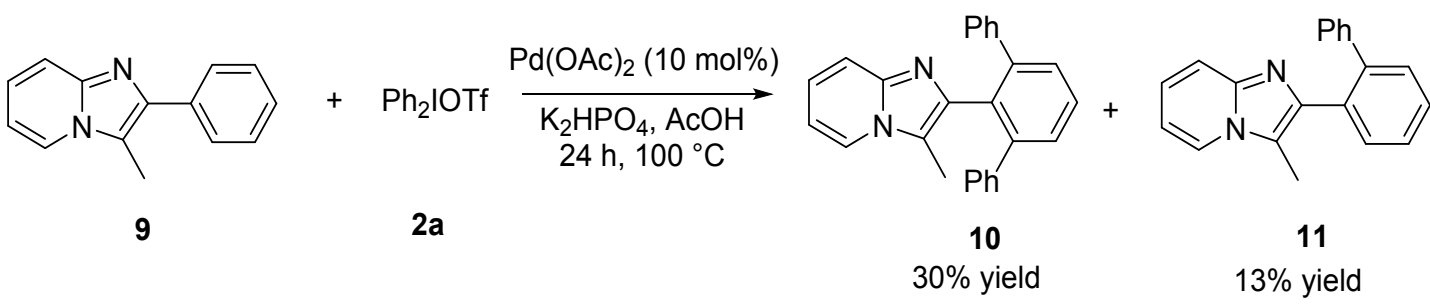

To an oven-dried Schlenk tube was added compound 9 (1.0 equiv.) in the presence of 3.0 equivalent of diaryliodonium salt 2a, 2.0 equivalent of $\mathrm{K}_{2} \mathrm{HPO}_{4}$ and $10 \mathrm{~mol} \%$ of $\mathrm{Pd}(\mathrm{OAc})_{2}$. The mixture was stirred at $100{ }^{\circ} \mathrm{C}$ in an oil bath. After 24 hours, the reaction mixture was filtered and the residue was washed with Sodium bicarbonate solution $(2 \times 50 \mathrm{~mL})$, then dried under vacuum and after column chromatography on silica gel afforded $21.6 \mathrm{mg}(30 \%)$ of $\mathbf{1 0}$ and $17.1 \mathrm{mg}(13 \%)$ of $\mathbf{1 1}$.

\section{3-Methyl-2-phenylimidazo[1,2- $\alpha]$ pyridine $(9)^{6}$}

${ }^{1} \mathbf{H}$ NMR (400 MHz, $\left.\mathbf{C D C l}_{3}\right) \delta 7.86(\mathrm{dd}, J=6.9,1.0 \mathrm{~Hz}, 1 \mathrm{H}), 7.80(\mathrm{dd}, J=8.2,1.2 \mathrm{~Hz}, 2 \mathrm{H}), 7.63(\mathrm{~d}, J$ $=9.1 \mathrm{~Hz}, 1 \mathrm{H}), 7.46(\mathrm{dd}, J=10.6,4.7 \mathrm{~Hz}, 2 \mathrm{H}), 7.38-7.31(\mathrm{~m}, 1 \mathrm{H}), 7.15(\mathrm{dd}, J=8.3,7.4 \mathrm{~Hz}, 1 \mathrm{H}), 6.81$ (t, $J=6.8 \mathrm{~Hz}, 1 \mathrm{H}), 2.61$ (d, $J=0.9 \mathrm{~Hz}, 3 \mathrm{H}) .{ }^{13} \mathbf{C}$ NMR (101 MHz, $\left.\mathbf{C D C l}_{3}\right) \delta 144.44,142.53,134.97$, $128.59,128.43,127.42,123.55,122.92,117.51,115.98,112.08,9.72$.

\section{2-([1,1':3',1'-Terphenyl]-2'-yl)-3-methylimidazo[1,2- $\alpha]$ pyridine (10)}

${ }^{1}$ H NMR (400 MHz, $\left.\mathbf{C D C l}_{3}\right) \delta 7.56-7.50(\mathrm{~m}, 2 \mathrm{H}), 7.46(\mathrm{~s}, 1 \mathrm{H}), 7.44$ (d, $\left.J=1.2 \mathrm{~Hz}, 1 \mathrm{H}\right), 7.39$ (d, $J=$ $9.1 \mathrm{~Hz}, 1 \mathrm{H}), 7.18-7.13(\mathrm{~m}, 4 \mathrm{H}), 7.12-7.08(\mathrm{~m}, 6 \mathrm{H}), 7.02-6.95(\mathrm{~m}, 1 \mathrm{H}), 6.65(\mathrm{t}, J=6.7 \mathrm{~Hz}, 1 \mathrm{H}), 1.78$ (s, 3H). ${ }^{13}$ C NMR (101 MHz, CDCl $)$ ) $\delta$ 143.74, 143.46, 141.98, 141.22, 131.61, 129.33, 129.21, 128.41, $127.41,126.26,122.53,118.08,117.33,111.37,8.24$.

\section{2-([1,1'-Biphenyl]-2-yl)-3-methylimidazo[1,2-a]pyridine (11)}


${ }^{1}$ H NMR (400 MHz, $\left.\mathbf{C D C l}_{3}\right) \delta 7.73-7.69(\mathrm{~m}, 1 \mathrm{H}), 7.66(\mathrm{~d}, J=9.1 \mathrm{~Hz}, 1 \mathrm{H}), 7.63(\mathrm{~d}, J=6.8 \mathrm{~Hz}, 1 \mathrm{H})$, $7.50-7.43(\mathrm{~m}, 3 \mathrm{H}), 7.21-7.12(\mathrm{~m}, 6 \mathrm{H}), 6.77(\mathrm{td}, J=6.8,1.0 \mathrm{~Hz}, 1 \mathrm{H}), 1.69(\mathrm{~s}, 3 \mathrm{H}) .{ }^{13} \mathbf{C}$ NMR (101 MHz, $\left.\mathbf{C D C l}_{3}\right) \delta 144.30,142.27,141.78,141.52,133.12,132.07,130.10,129.43,128.40,128.08,127.45$, $126.47,123.25,122.73,117.39,117.03,111.98,8.48$. 


\section{Part 7. References}

1. Bielawski, M.; Zhu, M.; Olofsson, B. Adv. Synth. Catal. 2007, 349, 2610-2618.

2. Zhu, M.; Jalalian, N.; Olofsson, B. Synlett 2008, 4, 592-596.

3. Bigot, A.; Williamson, A. E.; Gaunt, M. J. J. Am. Chem. Soc. 2011, 133, 13778-13781.

4. Bielawski, M.; Aili, D.; Olofsson, B. J. Org. Chem. 2008, 73, 4602-4607.

5. Ghosh, R.; Olofsson, B. Org. Lett. 2014, 16, 1830-1832.

6. Takizawa, S.-y.; Nishida, J.-i.; Tsuzuki, T.; Tokito, S.; Yamashita, Y. Inorg. Chem. 2007, 46, 43084319.

7. Banerji, B.; Chatterjee, S.; Chandrasekhar, K.; Bera, S.; Majumder, L.; Prodhan, C.; Chaudhuri, K. Org. Biomol. Chem. 2017, 15, 4130-4134. 
Part 8. X-Ray diffraction structural determination details

\section{Compound 3ca}

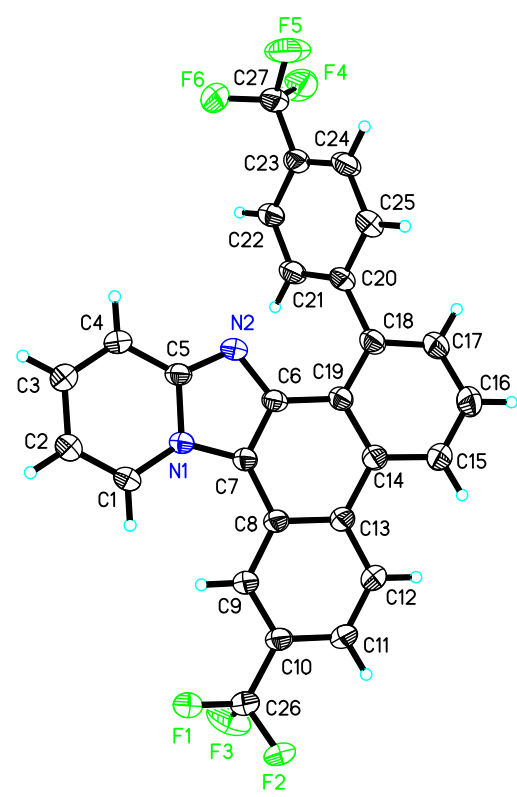

Crystal data and structure refinement for mo_d8v18572_0m.

Identification code

Empirical formula

Formula weight

Temperature

Wavelength

Crystal system

Space group

Unit cell dimensions

Volume

Z

Density (calculated)

Absorption coefficient

$\mathrm{F}(000)$

Crystal size mo_d8v18572_0m

C27 H14 F6 N2

480.40

296(2) K

$0.71073 \AA$

Monoclinic

P $21 / \mathrm{n}$

$a=8.566(3) \AA$

$\alpha=90^{\circ}$.

$\mathrm{b}=21.653(7) \AA$

$\beta=96.732(9)^{\circ}$.

$\mathrm{c}=22.398(6) \AA$

$\gamma=90^{\circ}$

4126(2) $\AA^{3}$

8

$1.547 \mathrm{Mg} / \mathrm{m}^{3}$

$0.129 \mathrm{~mm}^{-1}$

1952

$0.200 \times 0.170 \times 0.130 \mathrm{~mm}^{3}$ 
Theta range for data collection

Index ranges

Reflections collected

Independent reflections

Completeness to theta $=25.242^{\circ}$

Absorption correction

Max. and min. transmission

Refinement method

Data / restraints / parameters

Goodness-of-fit on $\mathrm{F}^{2}$

Final R indices [I $>2 \operatorname{sigma}(\mathrm{I})]$

$\mathrm{R}$ indices (all data)

Largest diff. peak and hole
2.461 to $25.999^{\circ}$.

$-10<=\mathrm{h}<=10,-26<=\mathrm{k}<=26,-27<=\mathrm{l}<=27$

44873

$8108[\mathrm{R}(\mathrm{int})=0.0500]$

$99.8 \%$

Semi-empirical from equivalents

0.7456 and 0.6055

Full-matrix least-squares on $\mathrm{F}^{2}$

8108 / 0 / 632

1.041

$\mathrm{R} 1=0.0584, w R 2=0.1575$

$\mathrm{R} 1=0.0876, \mathrm{wR} 2=0.1869$

0.403 and -0.308 e. $\AA^{-3}$ 
Part $9 .{ }^{1} \mathrm{H},{ }^{13} \mathrm{C}$ and ${ }^{19} \mathrm{~F}$ NMR spectra of products
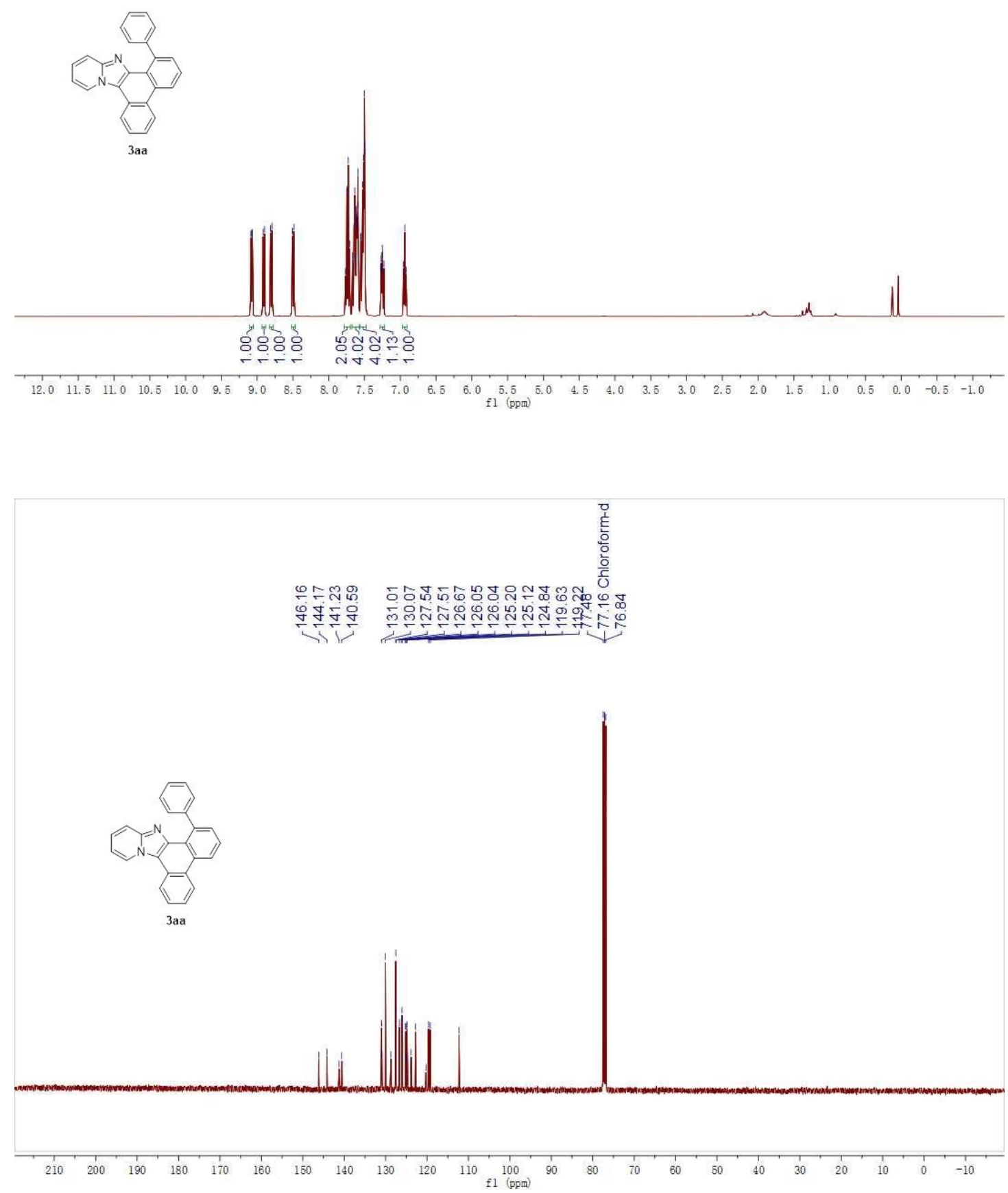

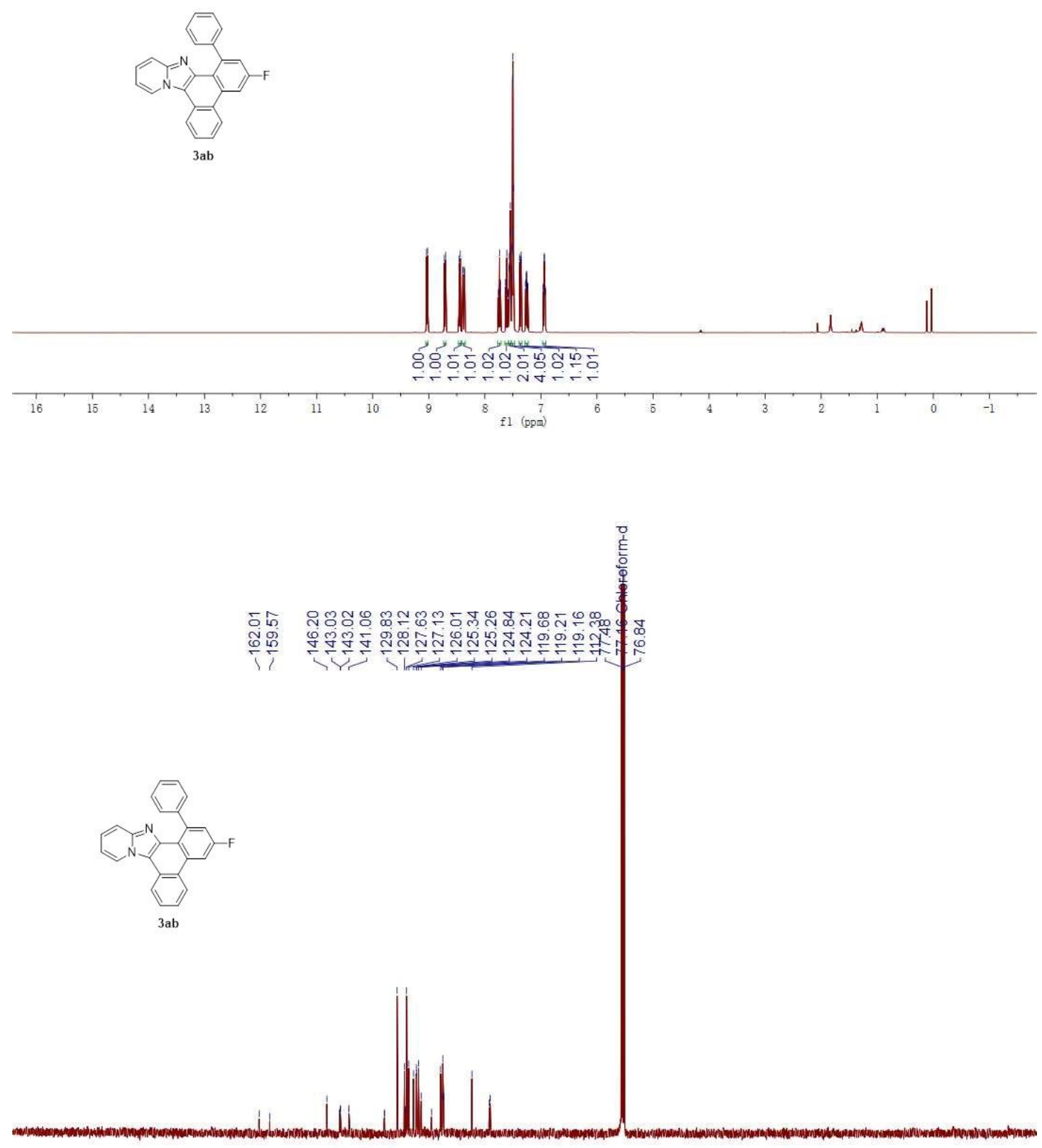

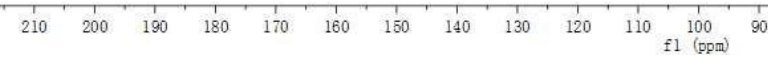




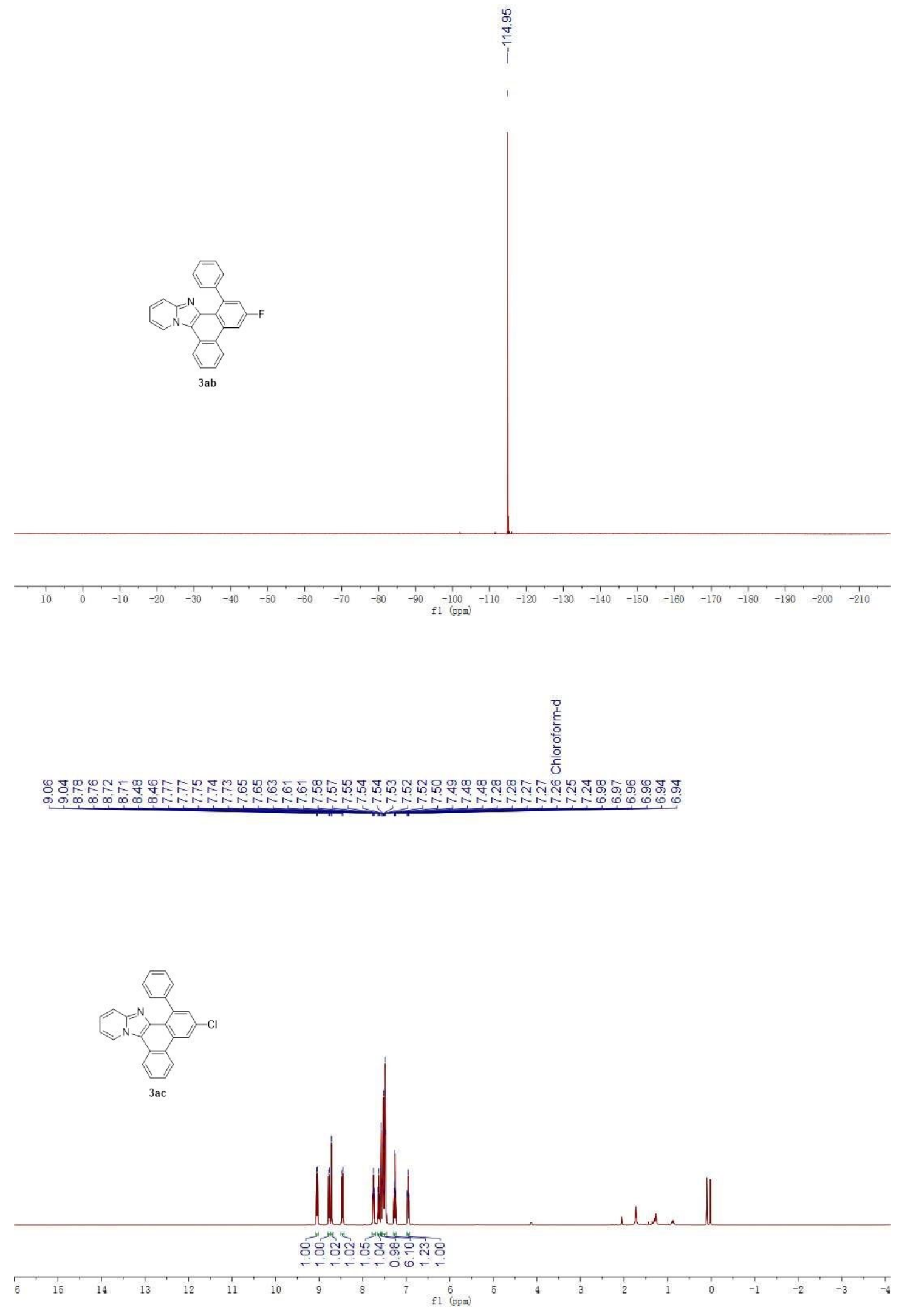



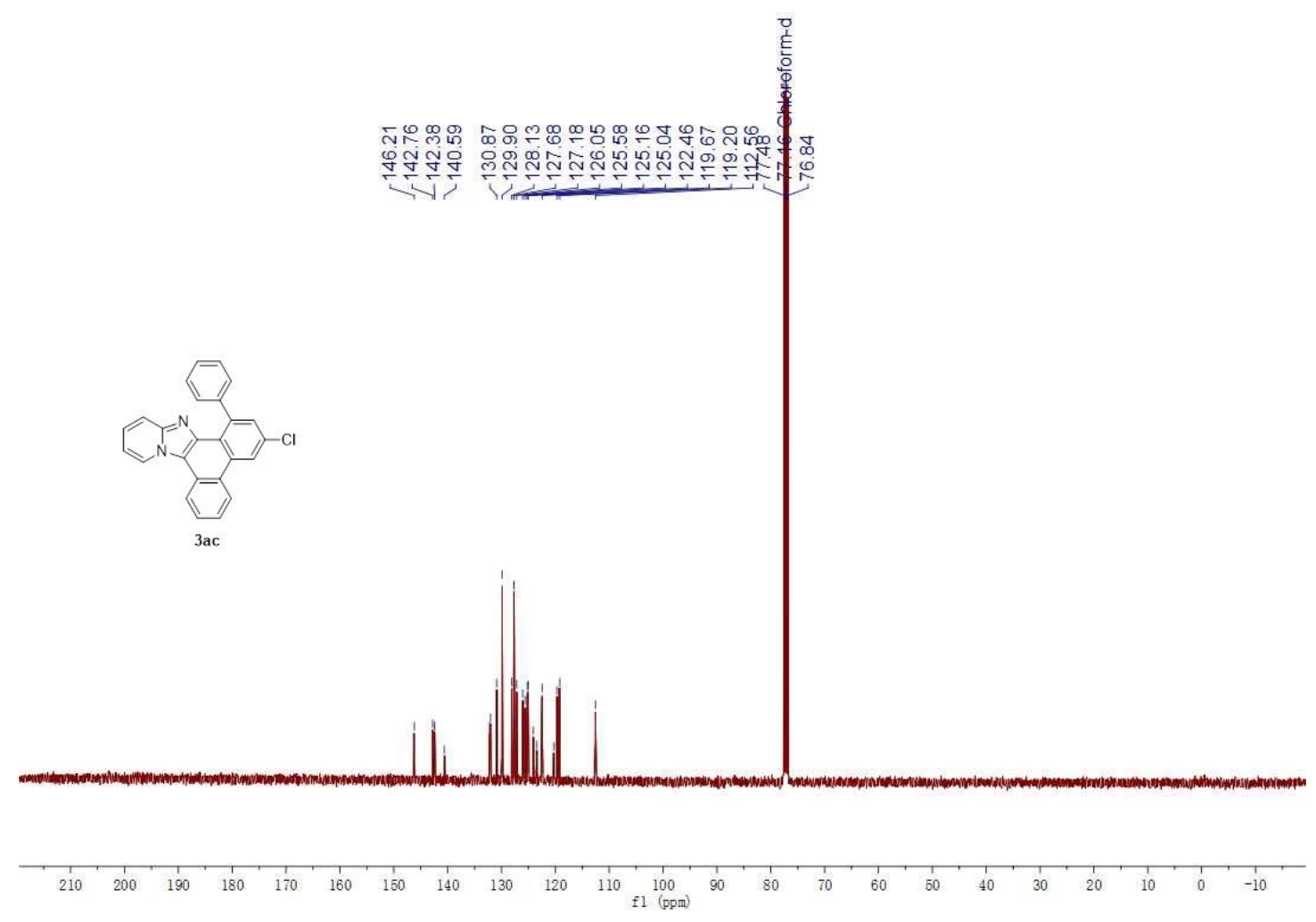

한

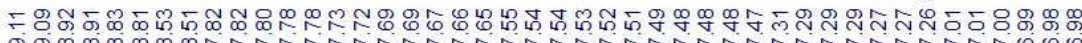

$+\infty$

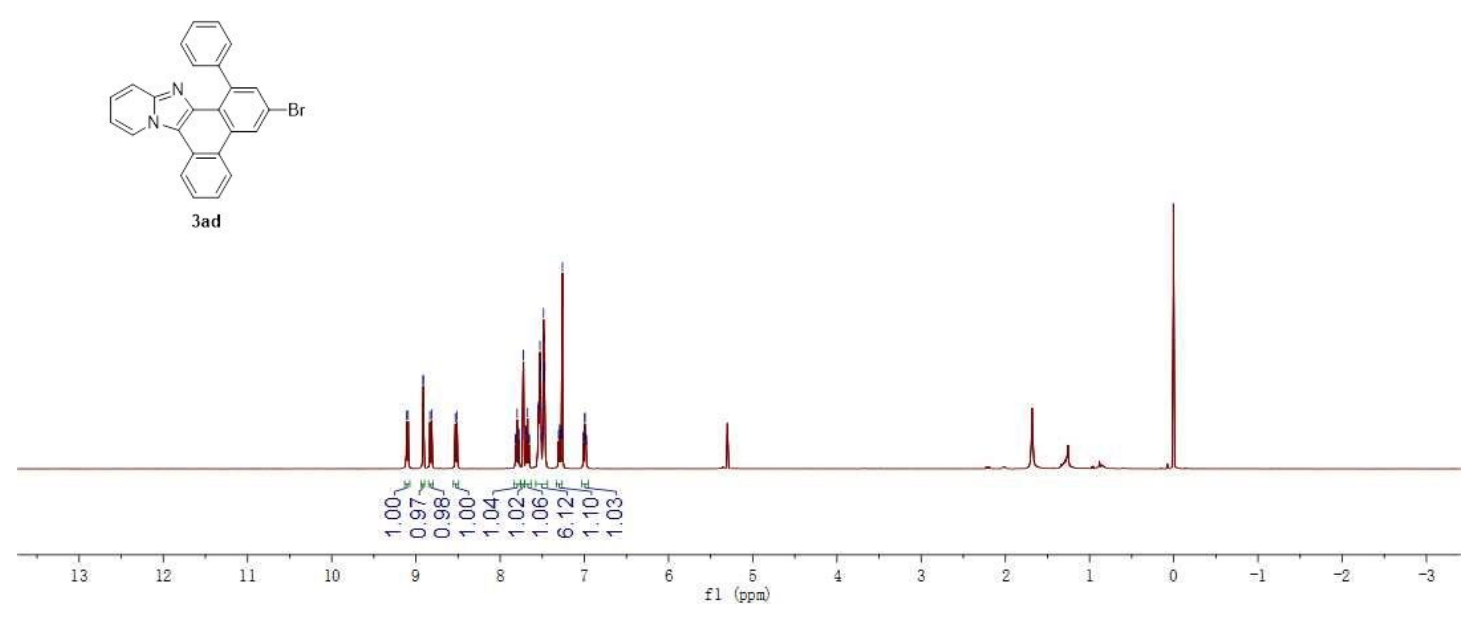



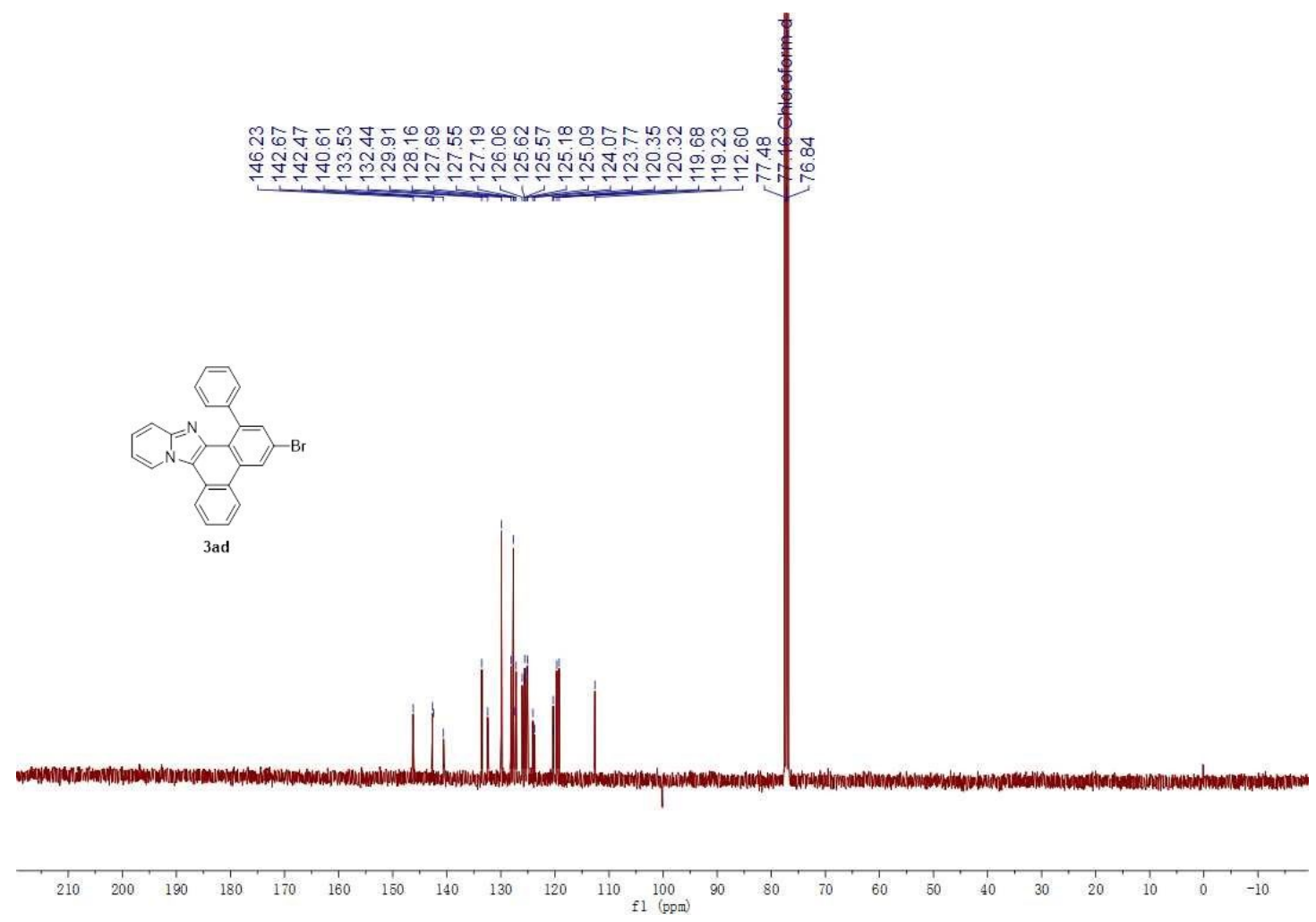

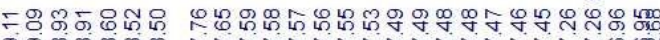

gon
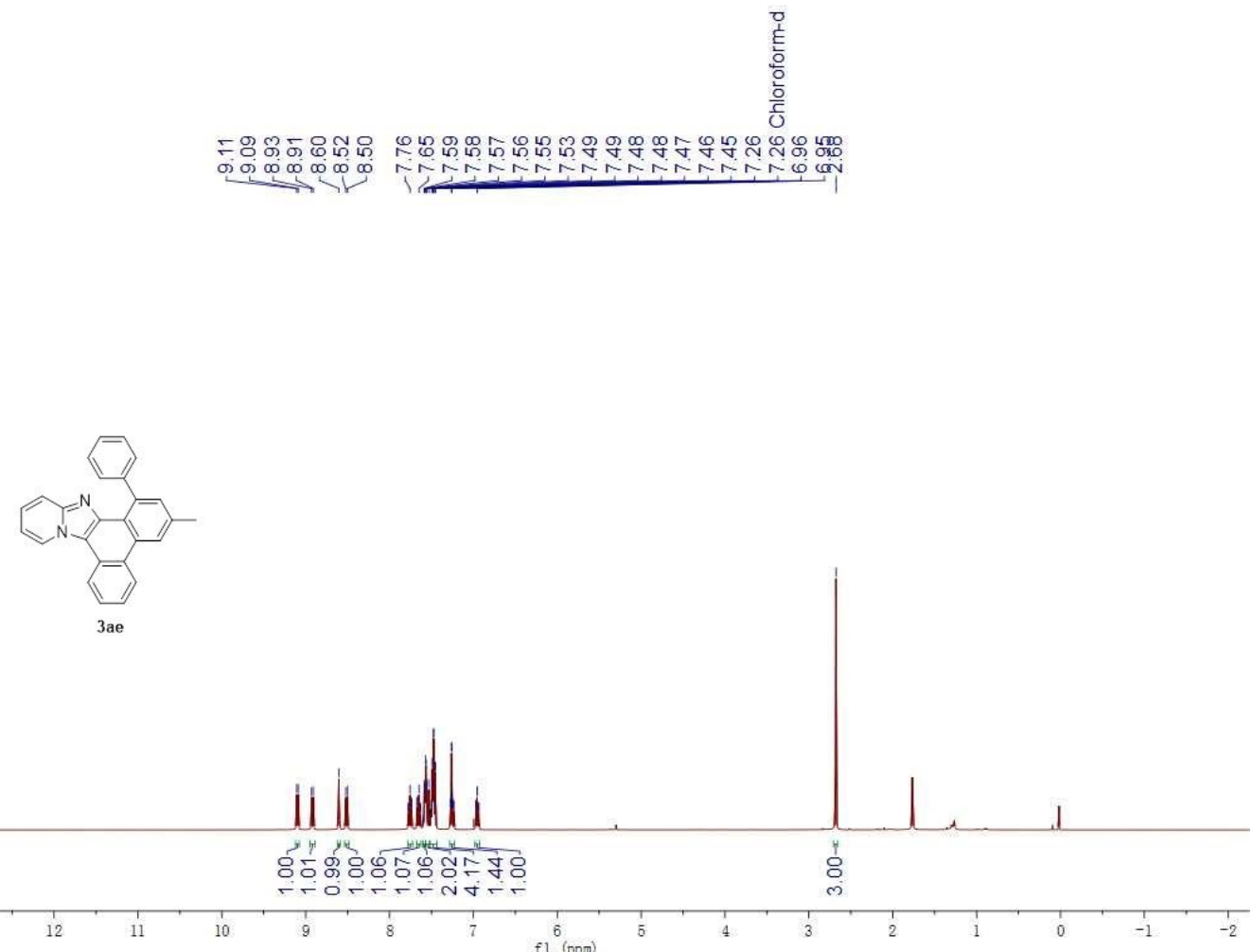


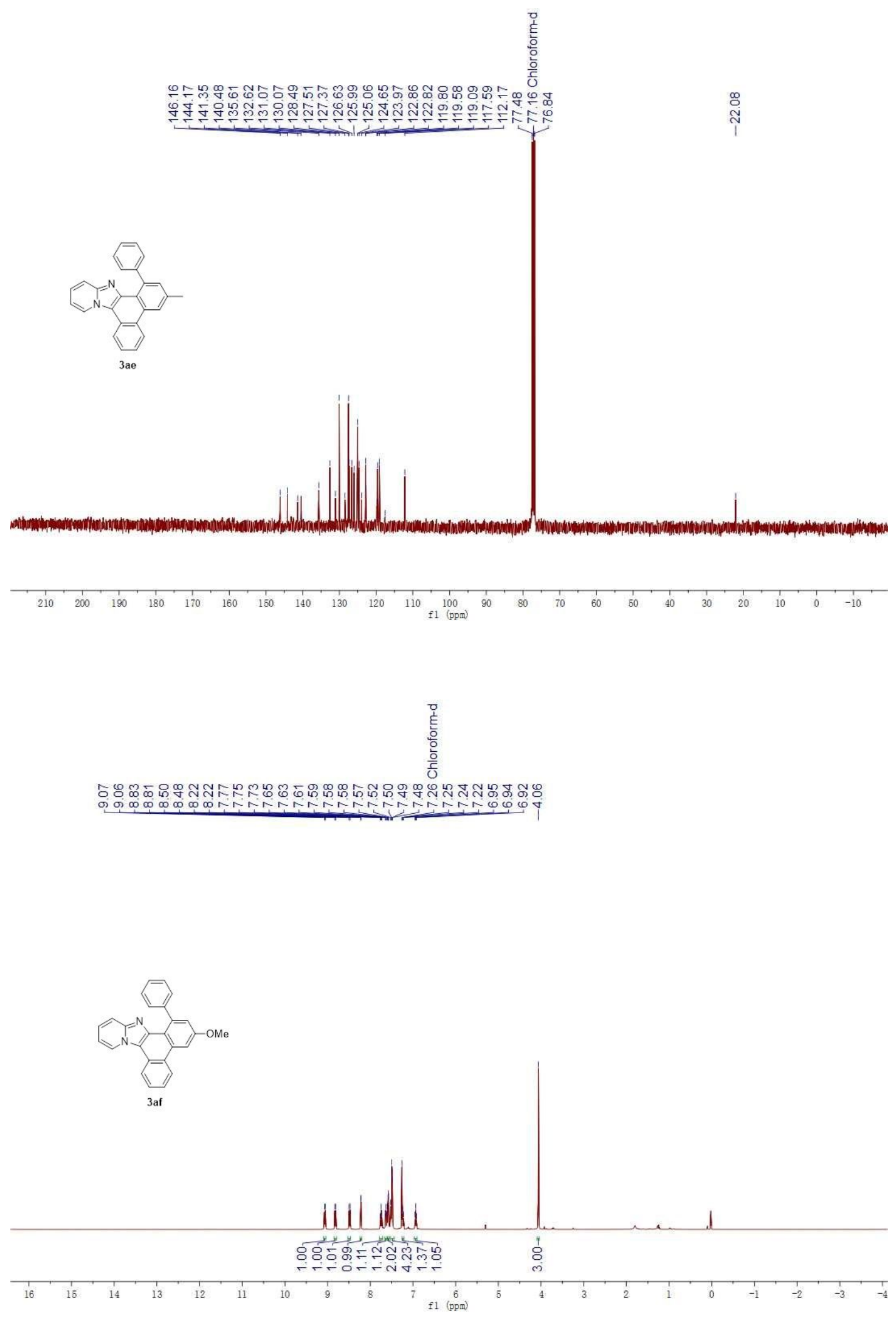



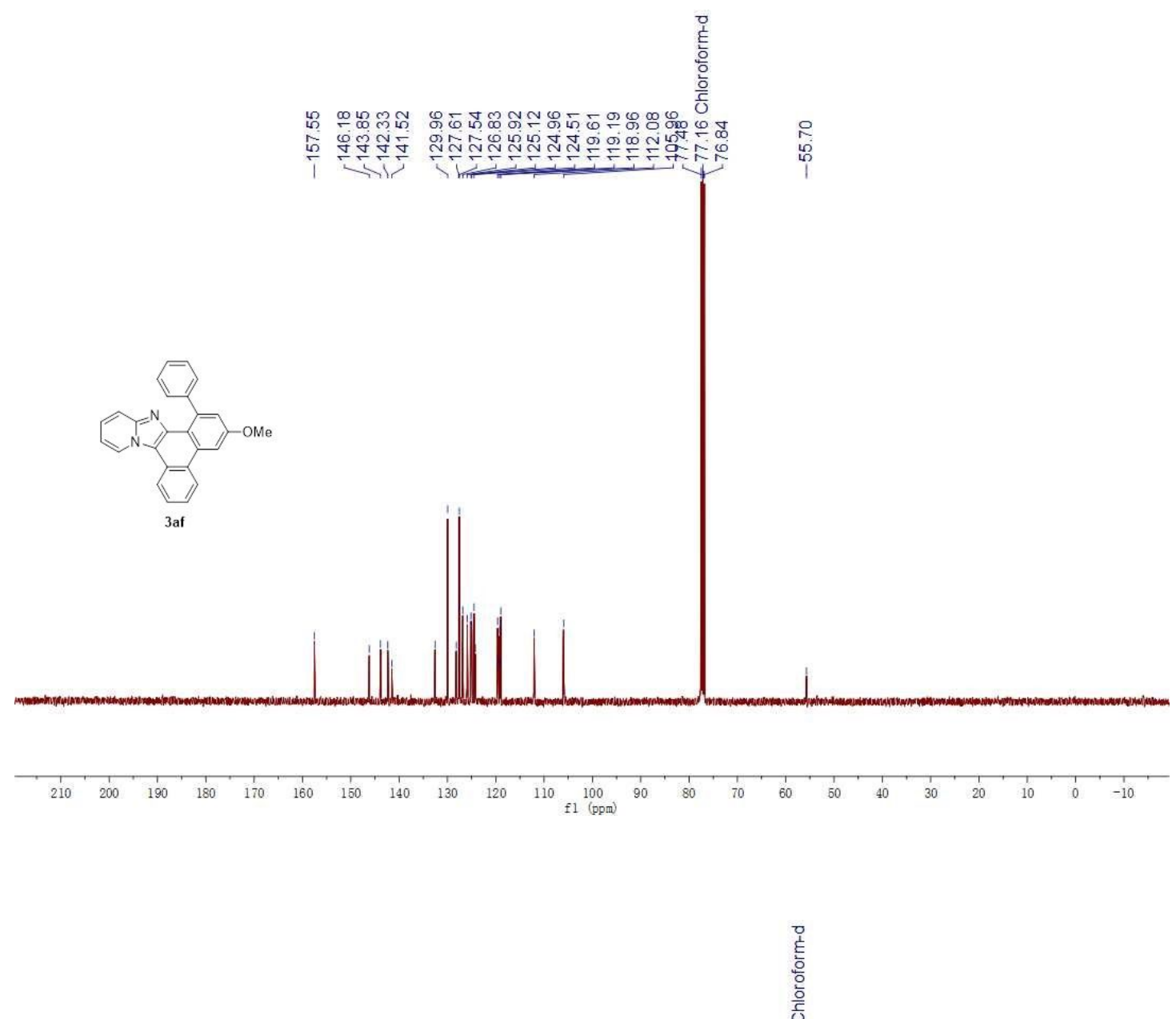

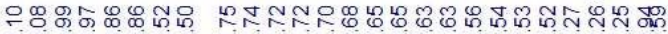

का $\infty 00000000$

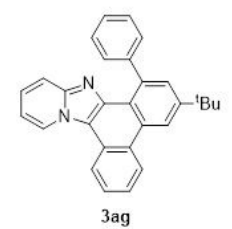

wh the

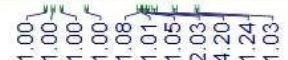

高

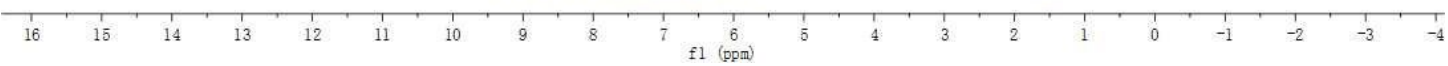




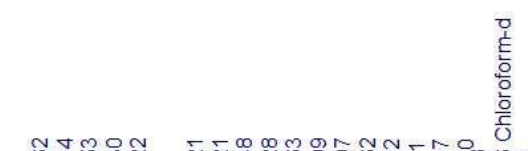

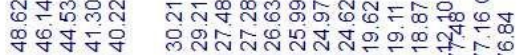

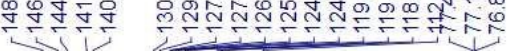

㐘只

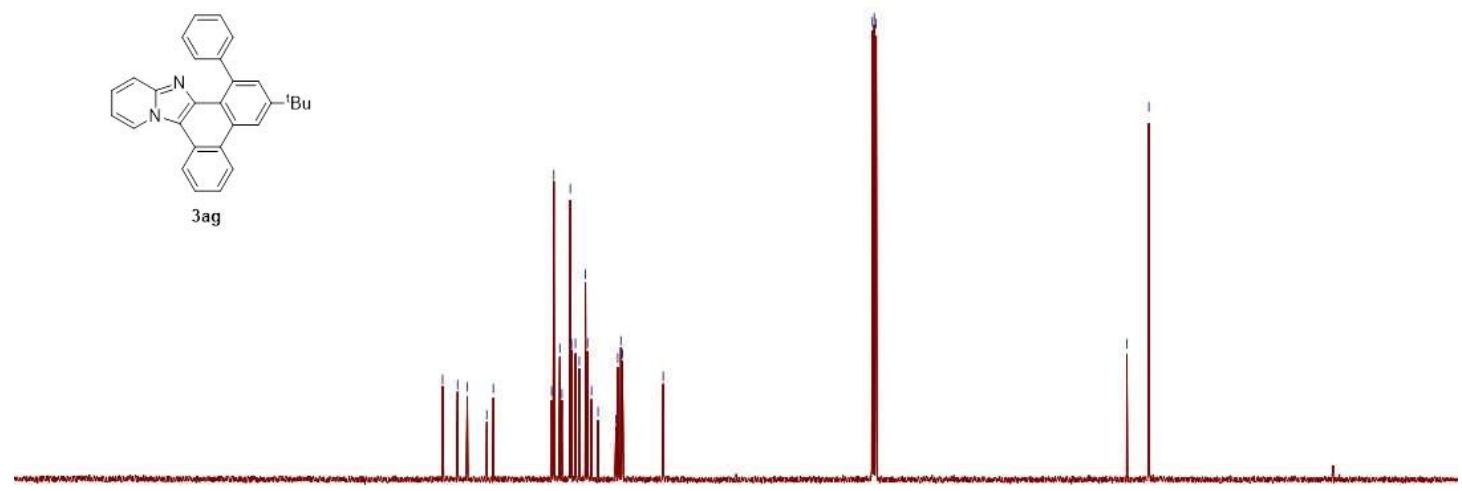

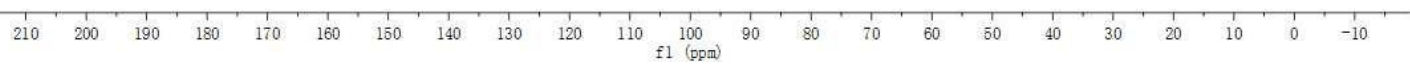

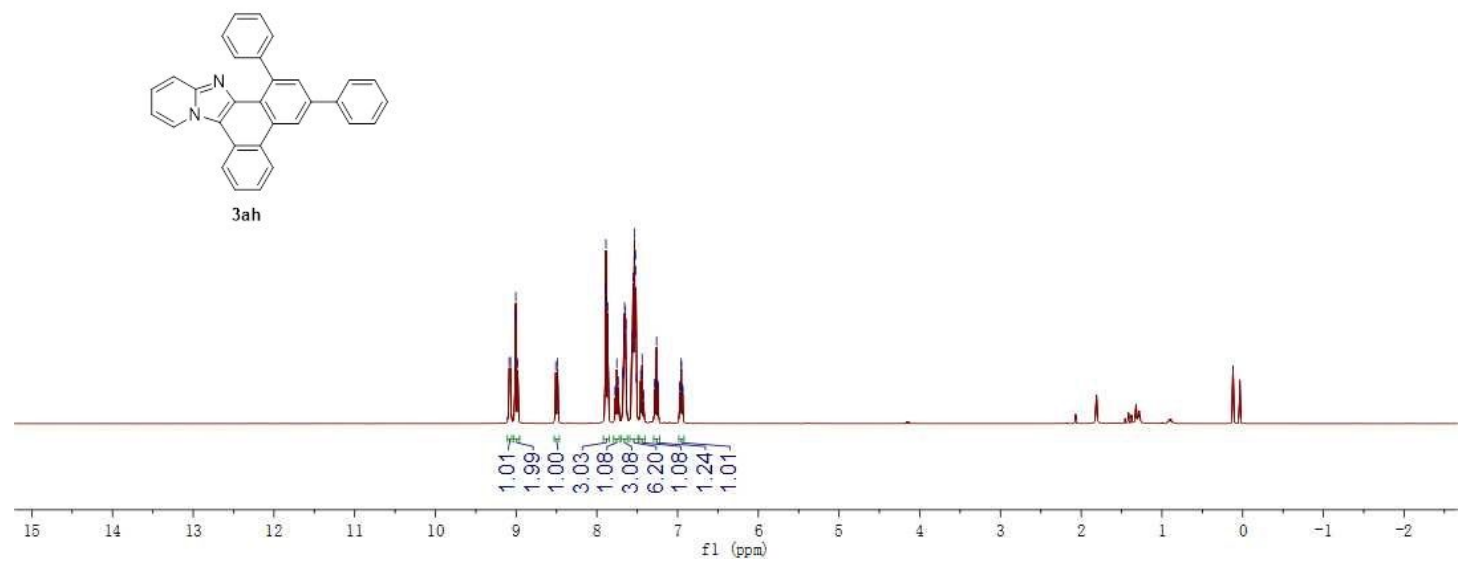



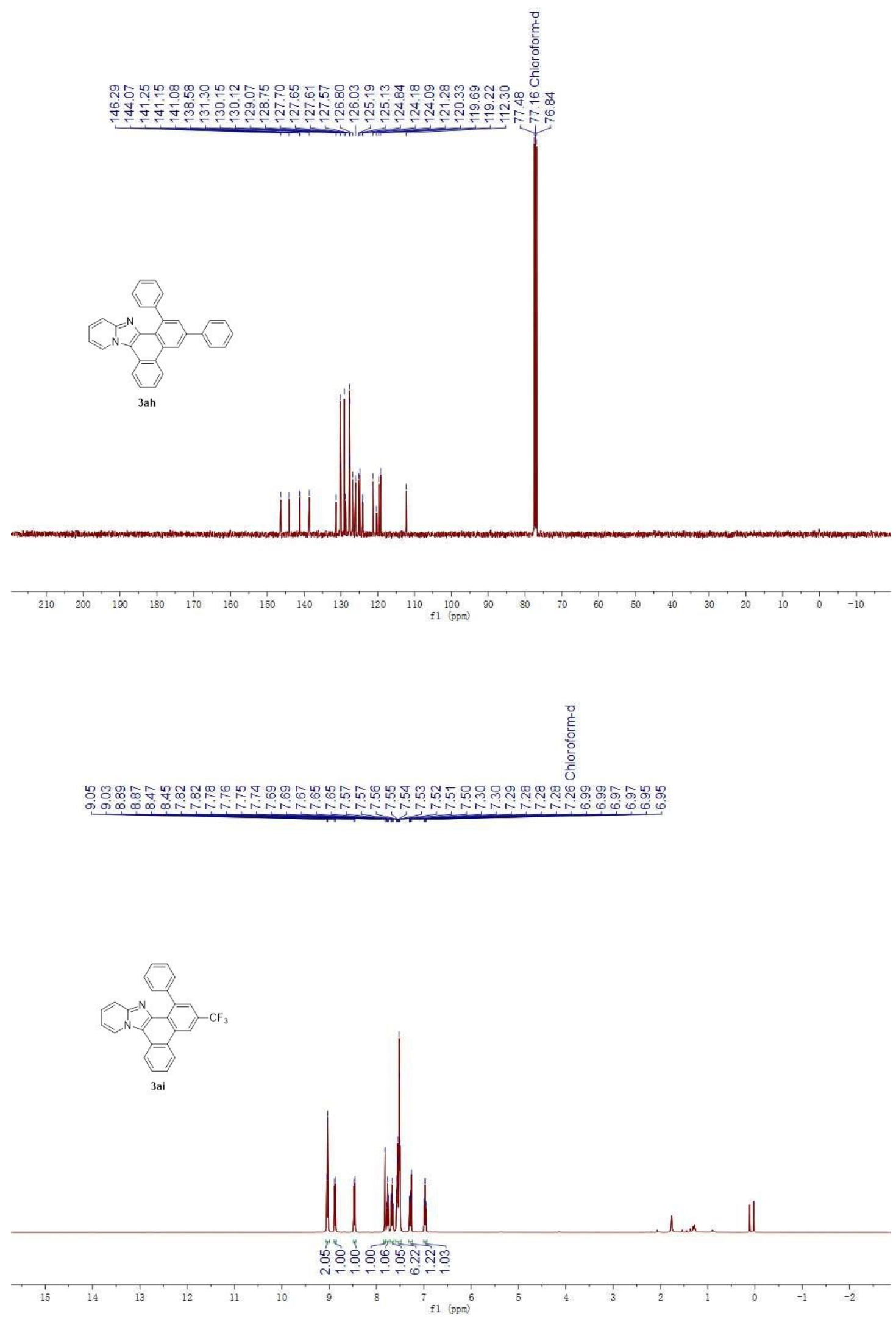

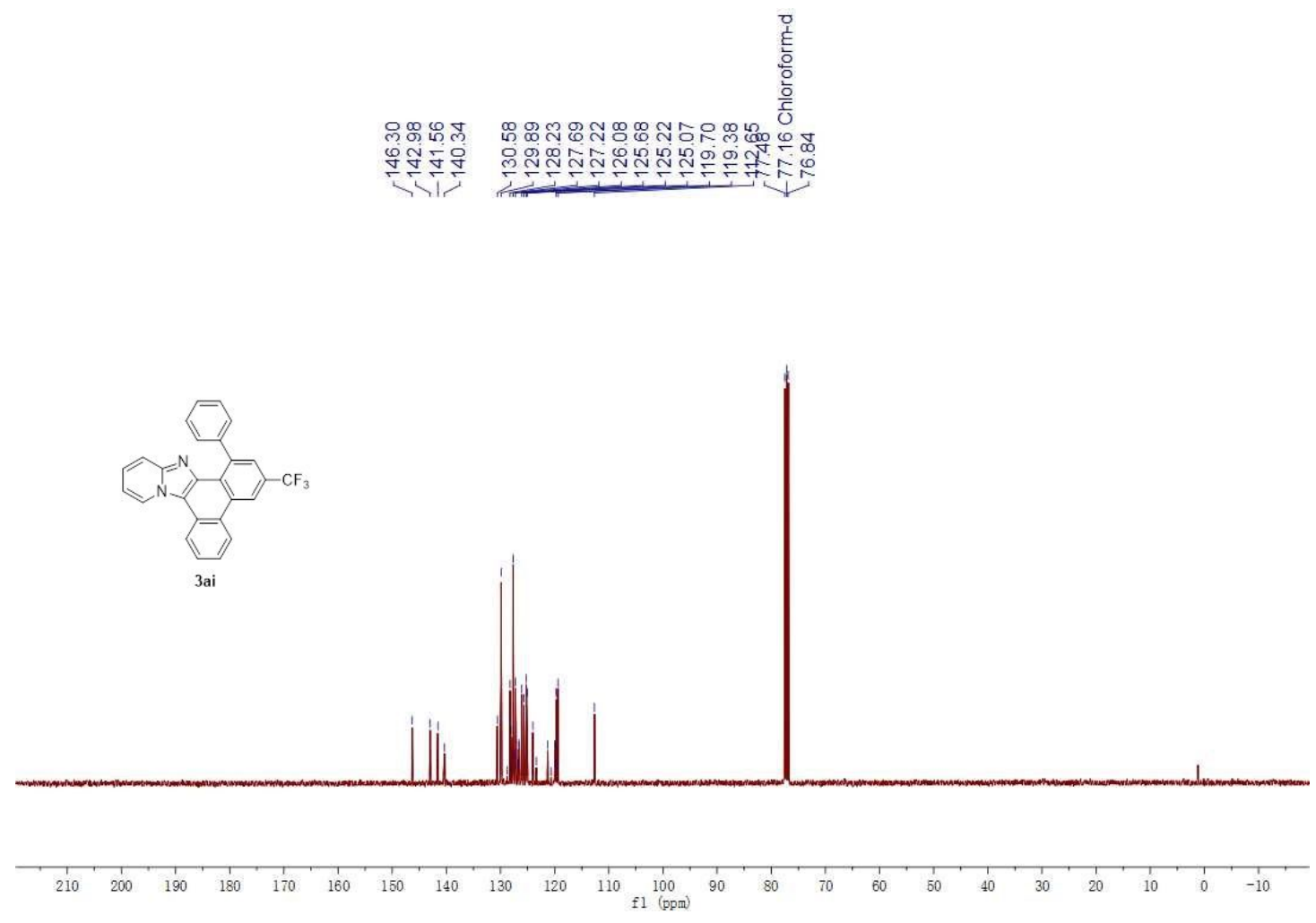

$\frac{0}{6}$

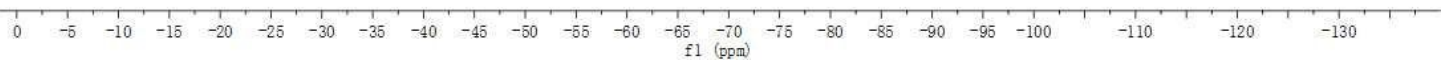




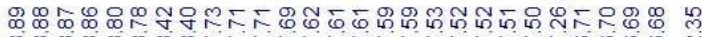

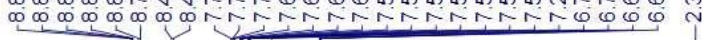

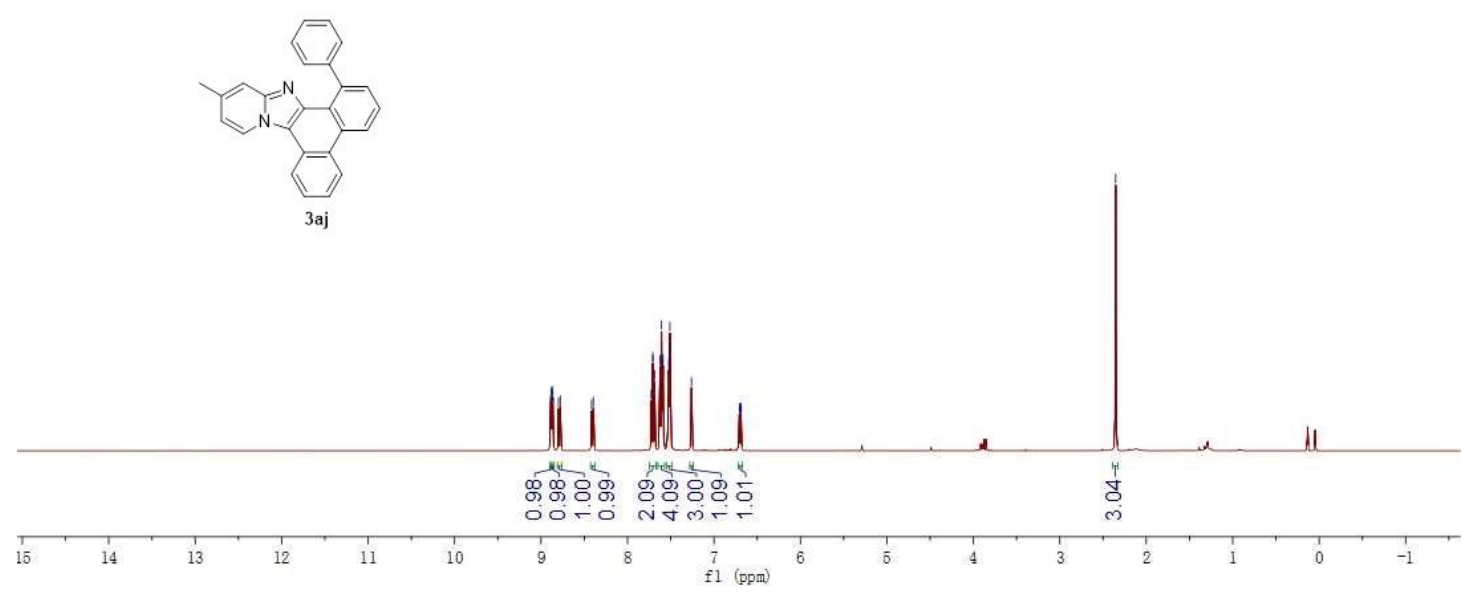

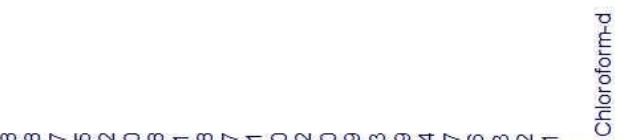

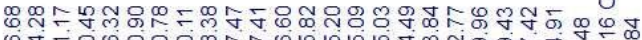

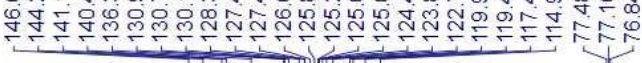

$\stackrel{\overline{\mathrm{m}}}{\stackrel{\mathrm{T}}{\mathrm{T}}}$
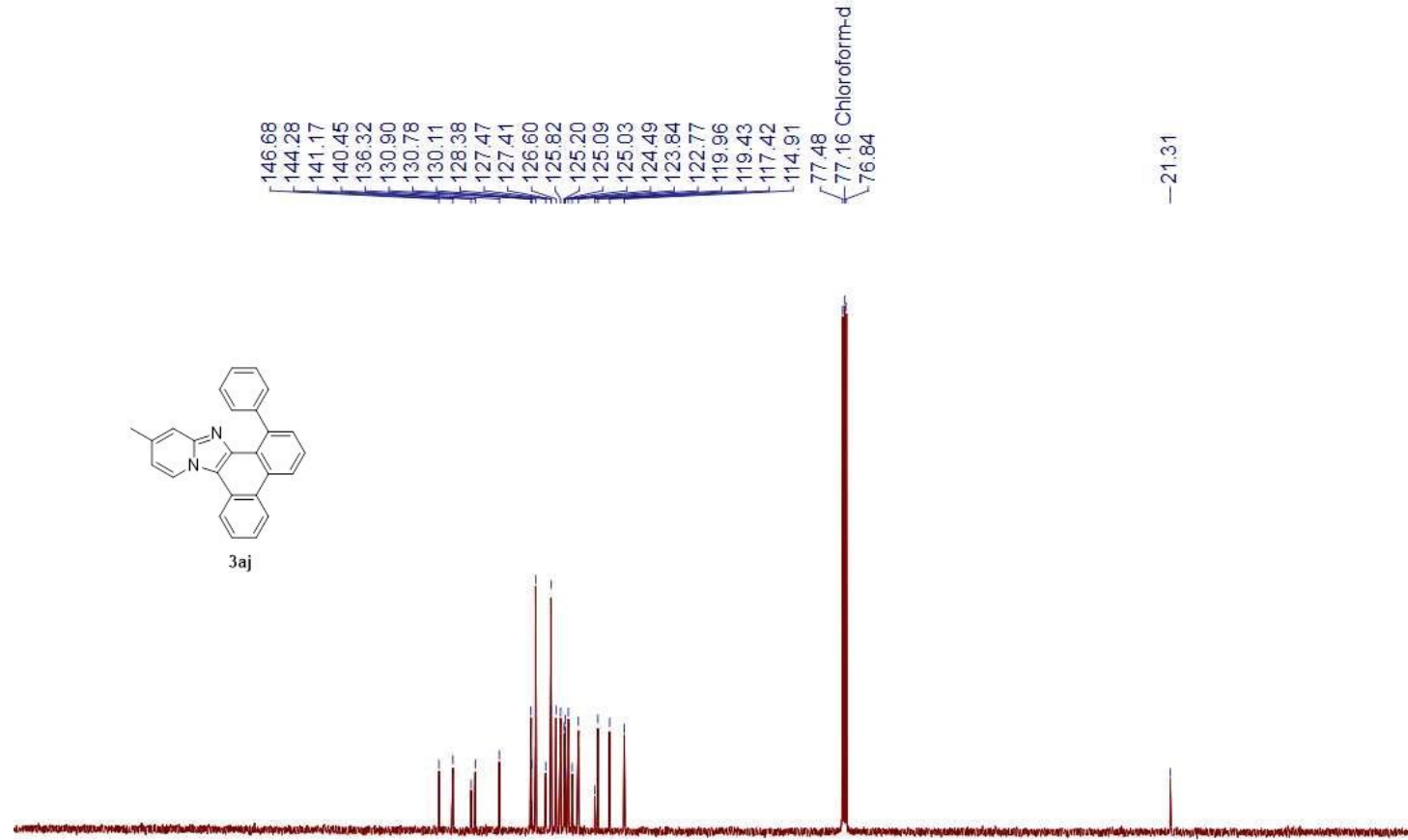

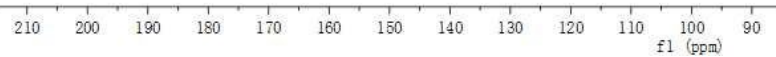



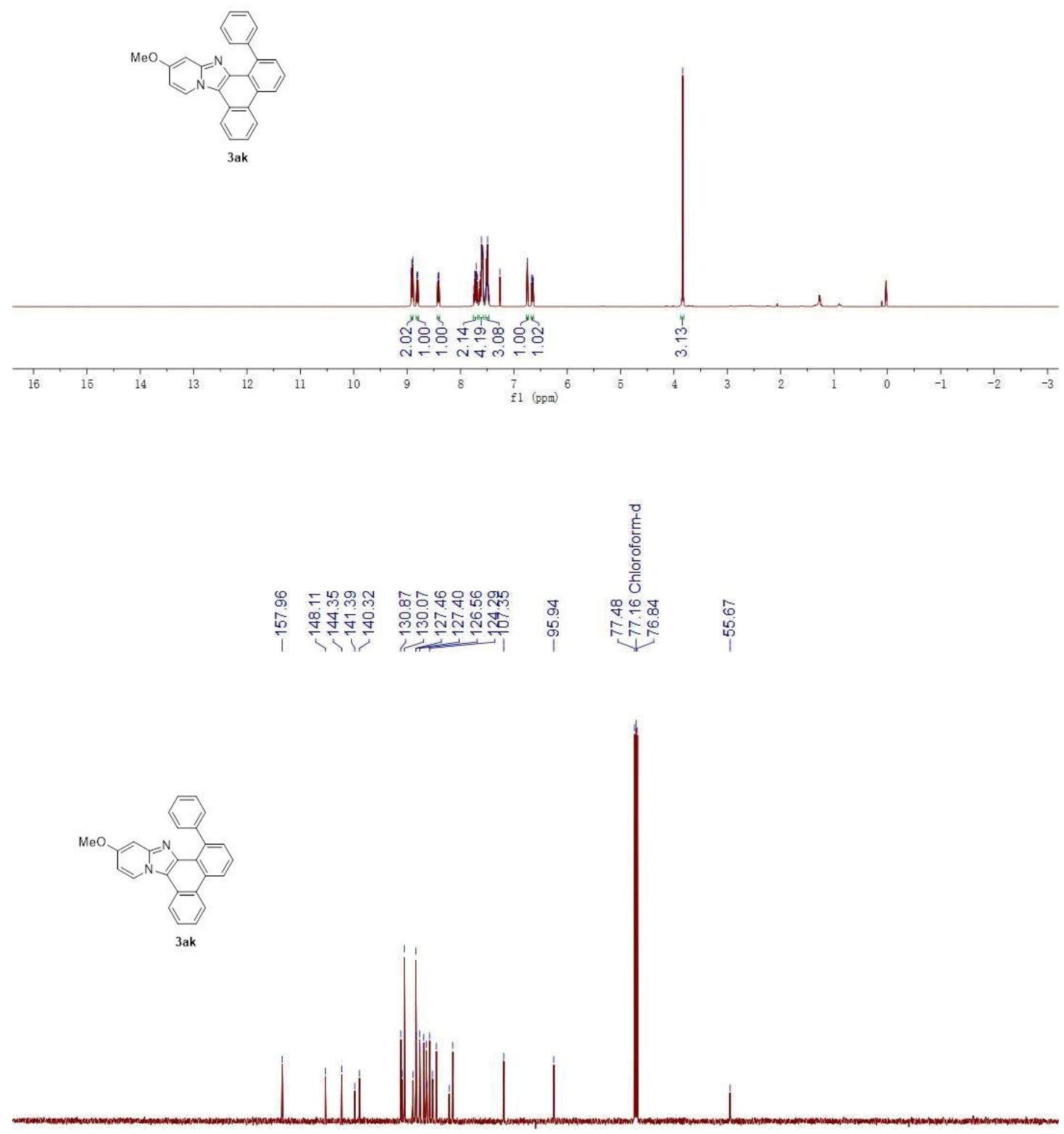

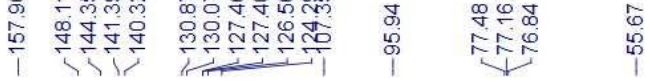

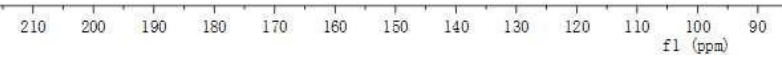


20
$\frac{10}{5}$

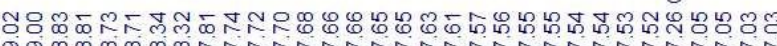

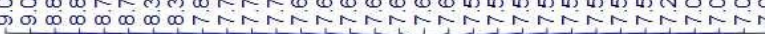

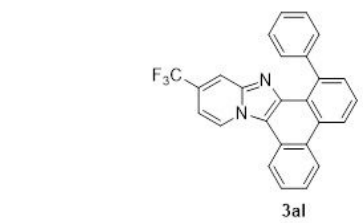

L the U

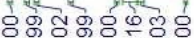

Lr
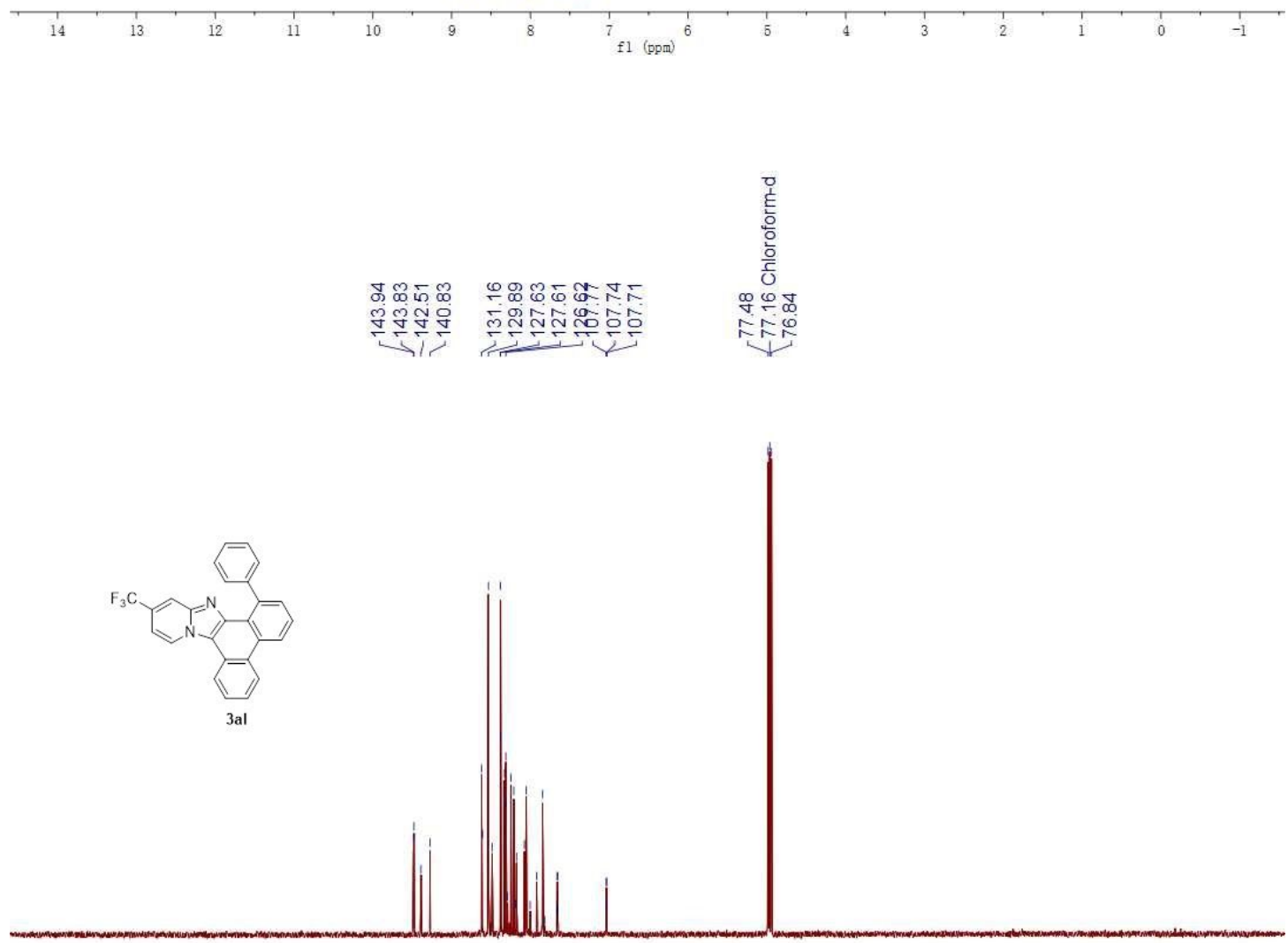

$\begin{array}{llllllllllllll}1 & 1 & 200 & 190 & 180 & 170 & 160 & 150 & 140 & 130 & 120 & 110 & 100 & 1 \\ \mathrm{f} 1 & (\mathrm{pgm}) & 90\end{array}$ 


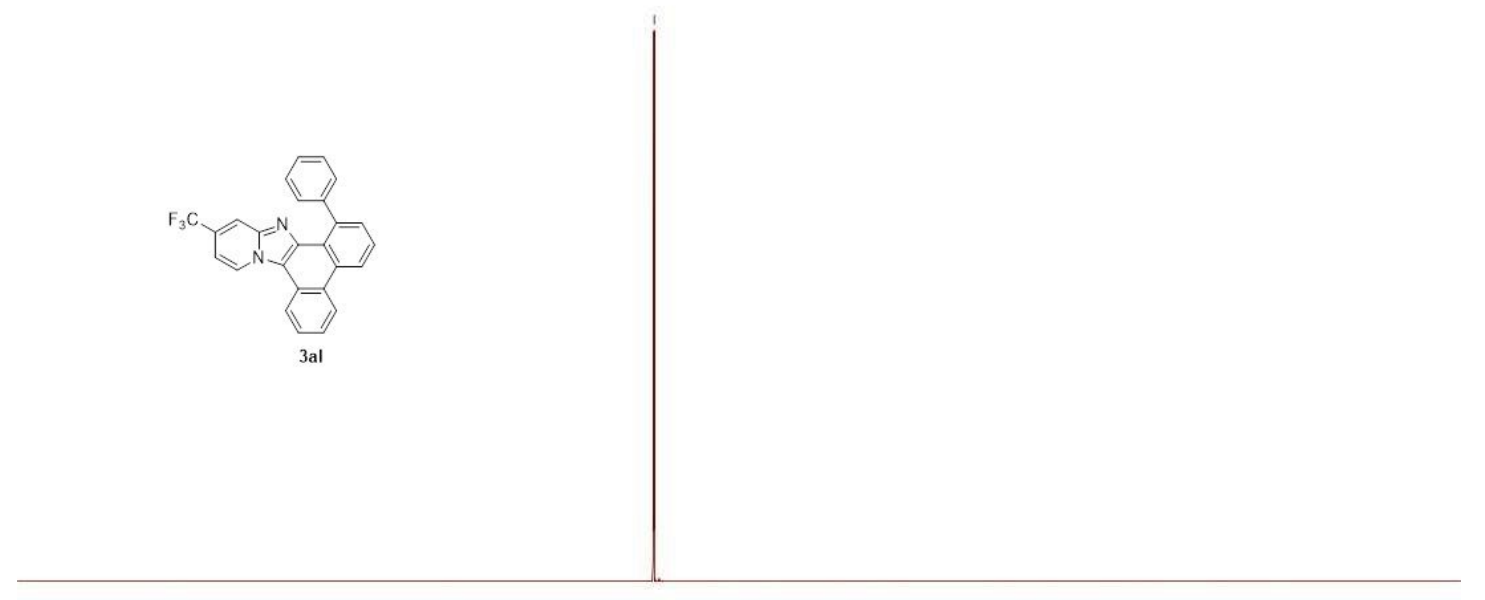

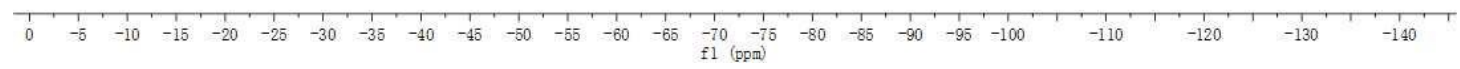

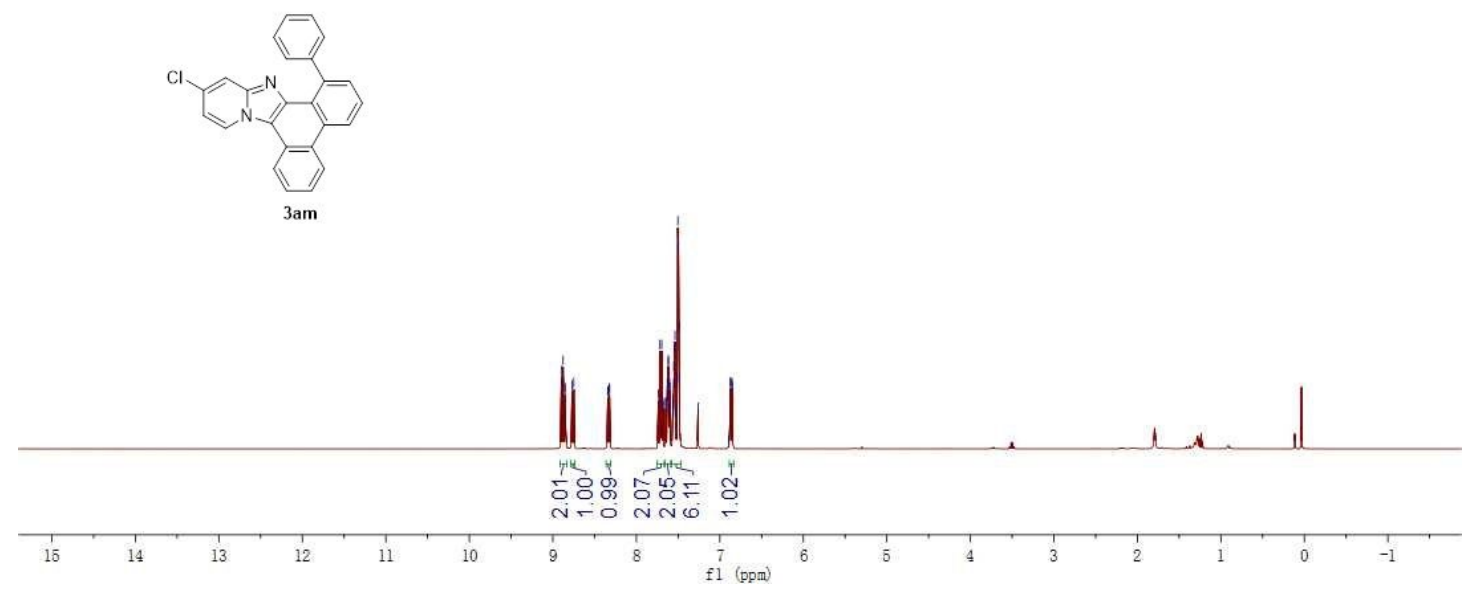



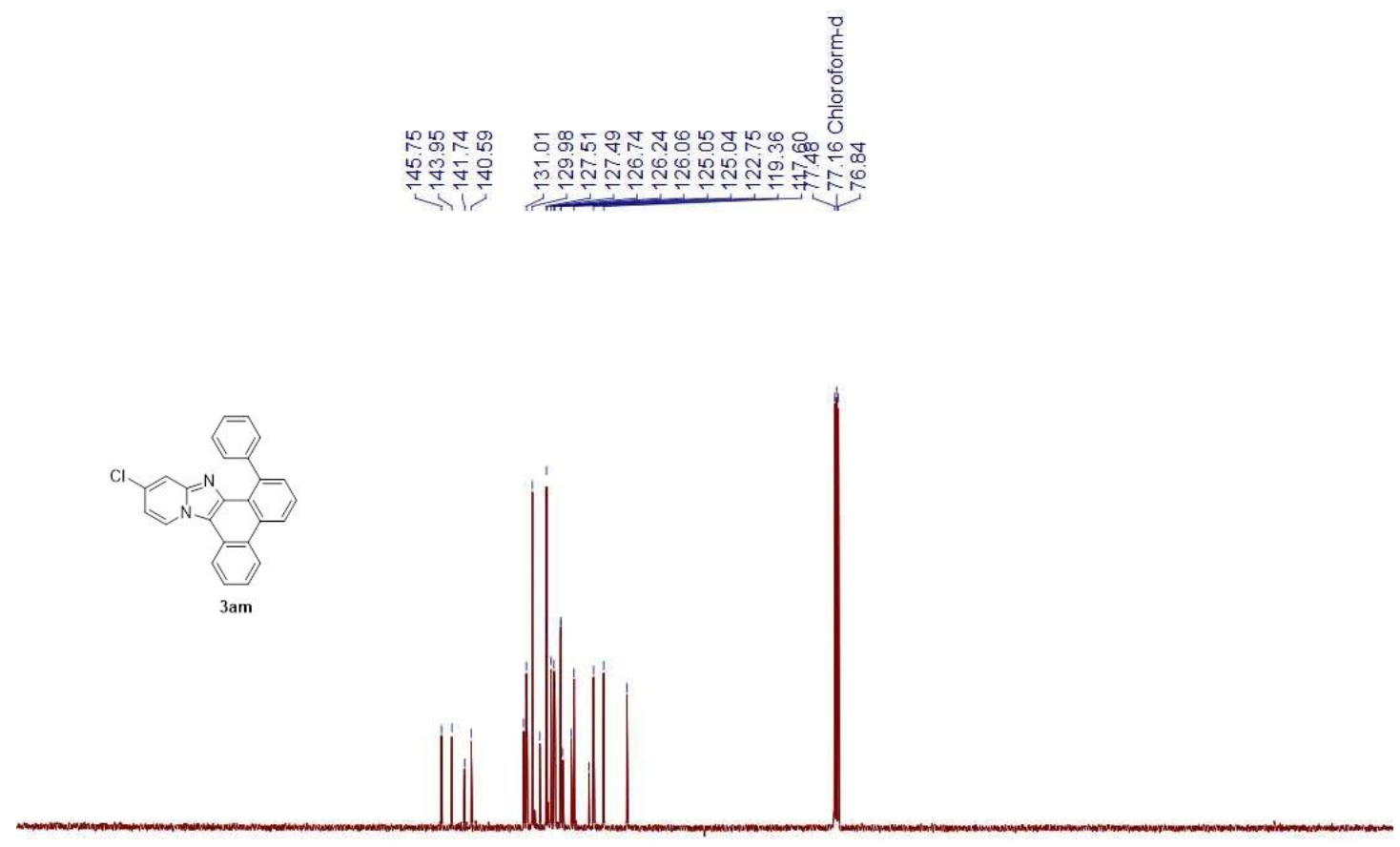

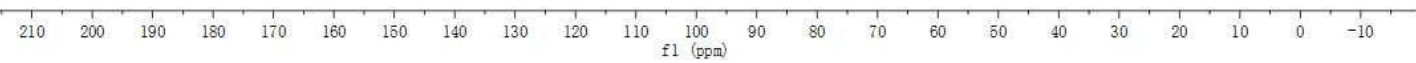

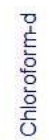

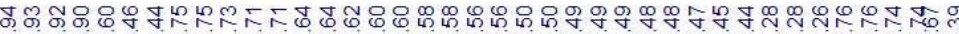

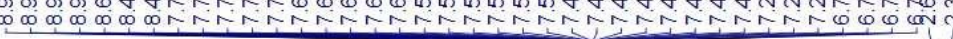

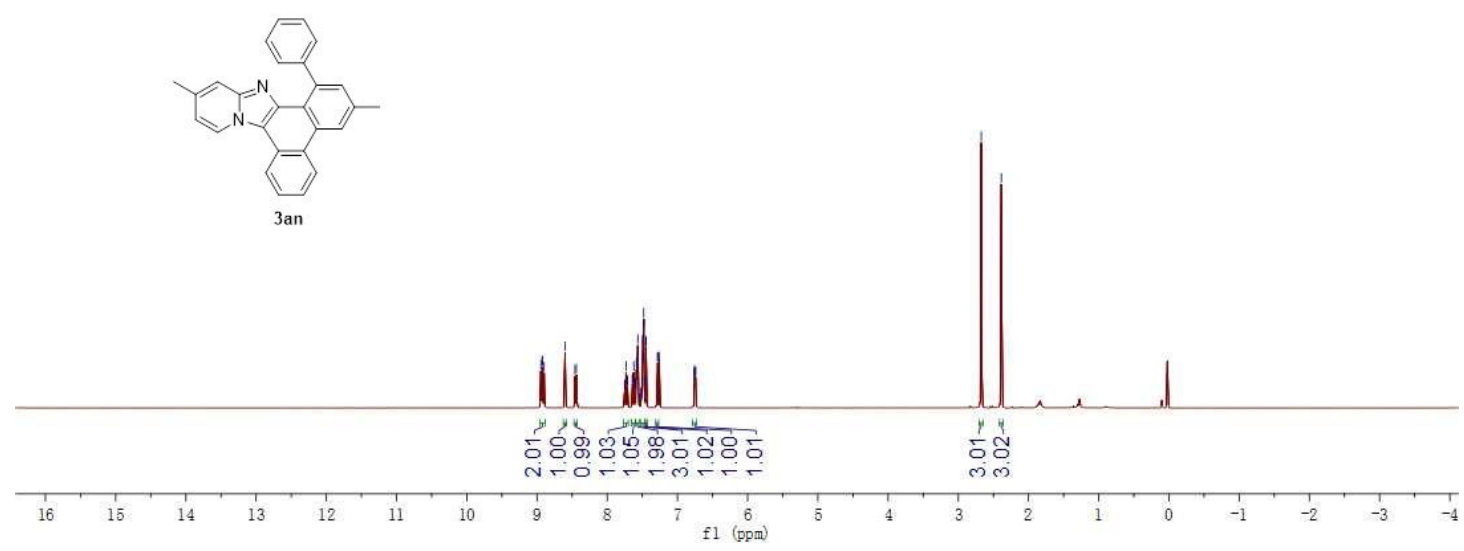



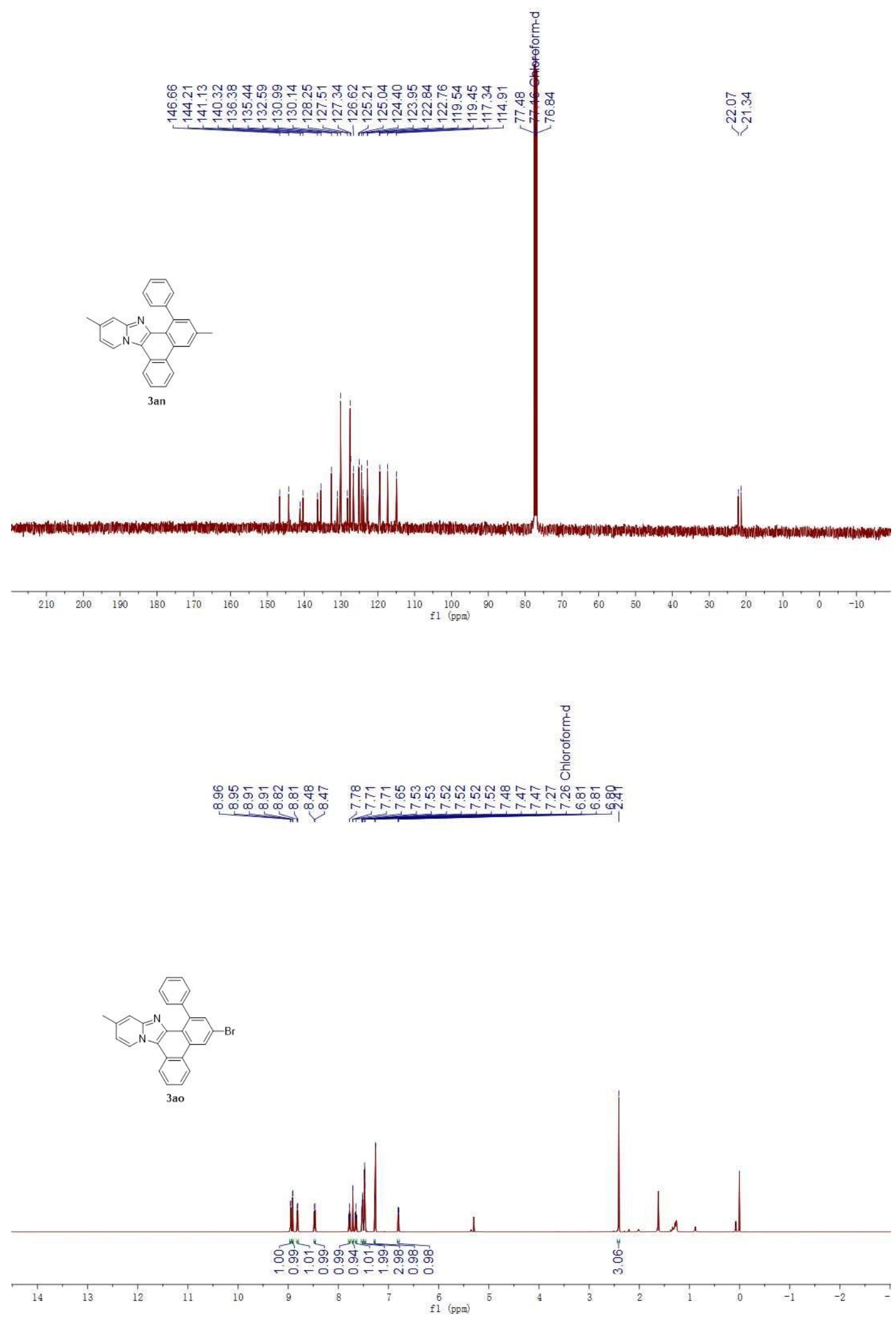


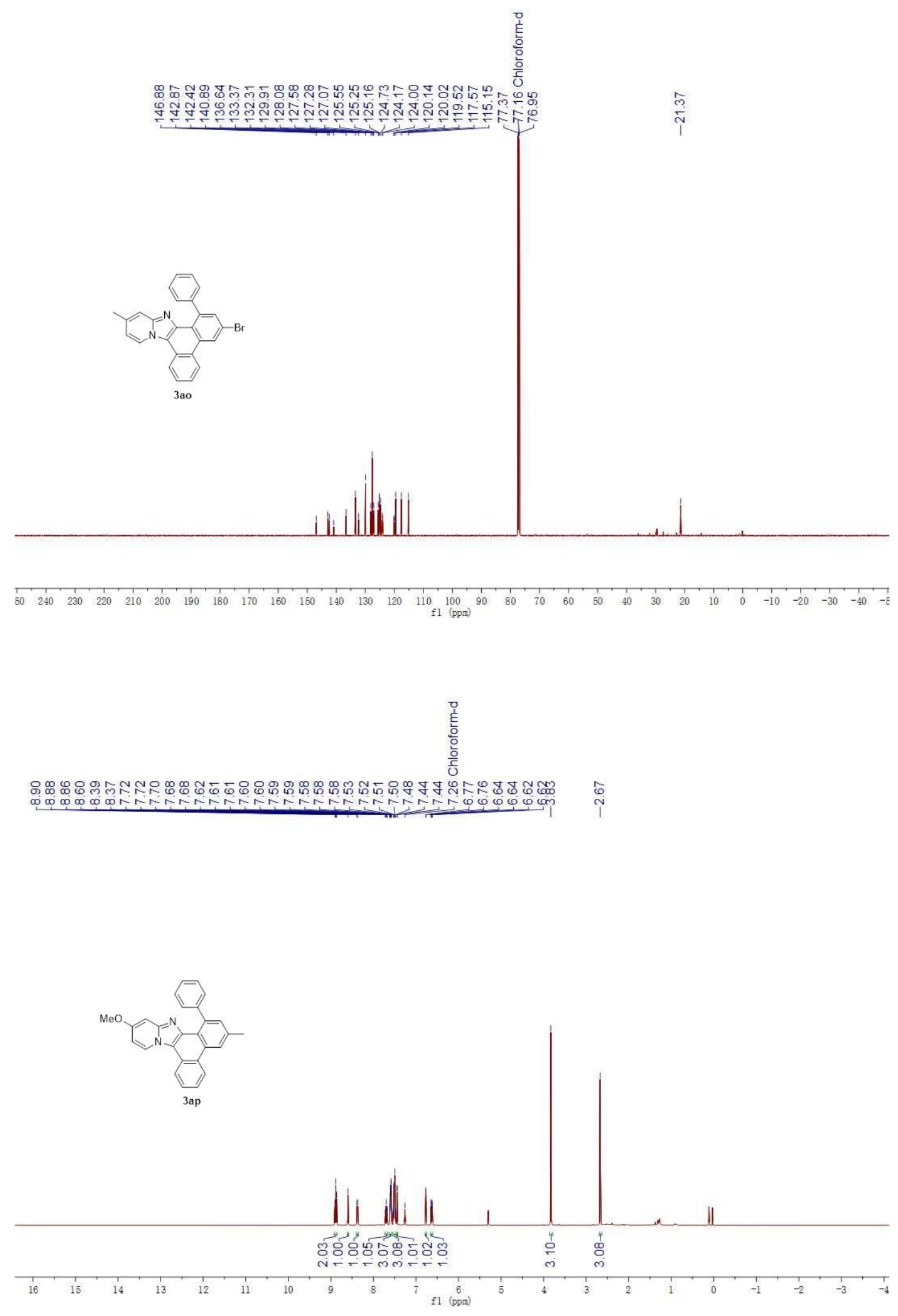




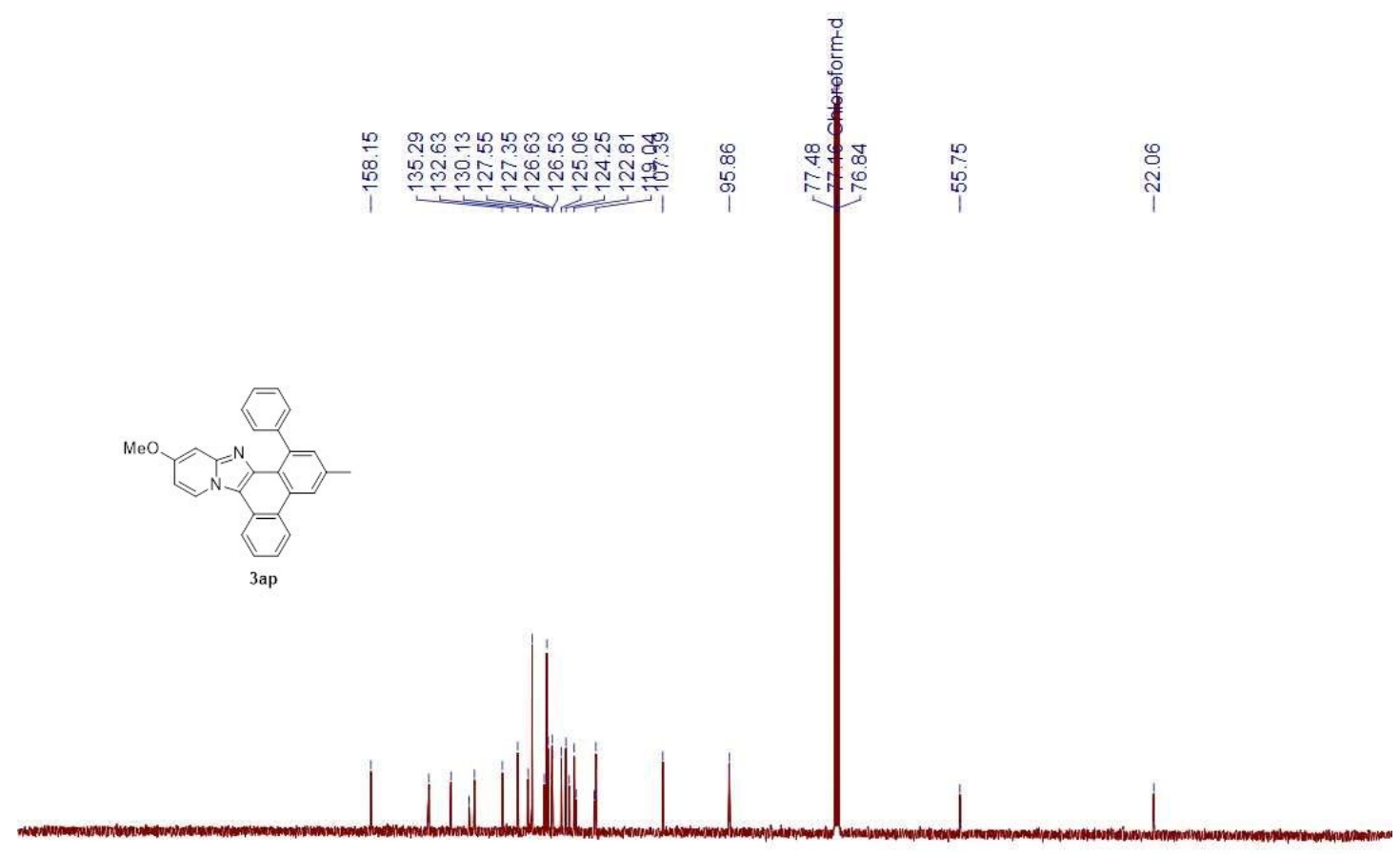

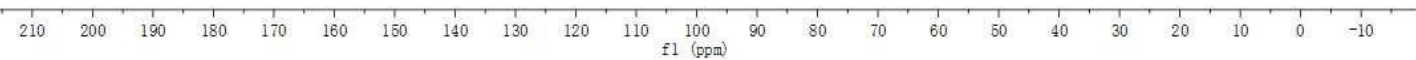

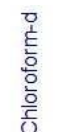

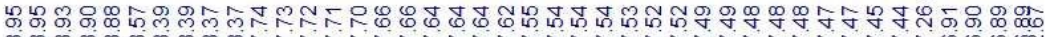

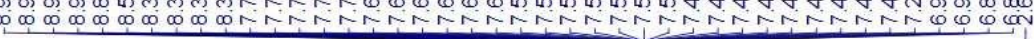

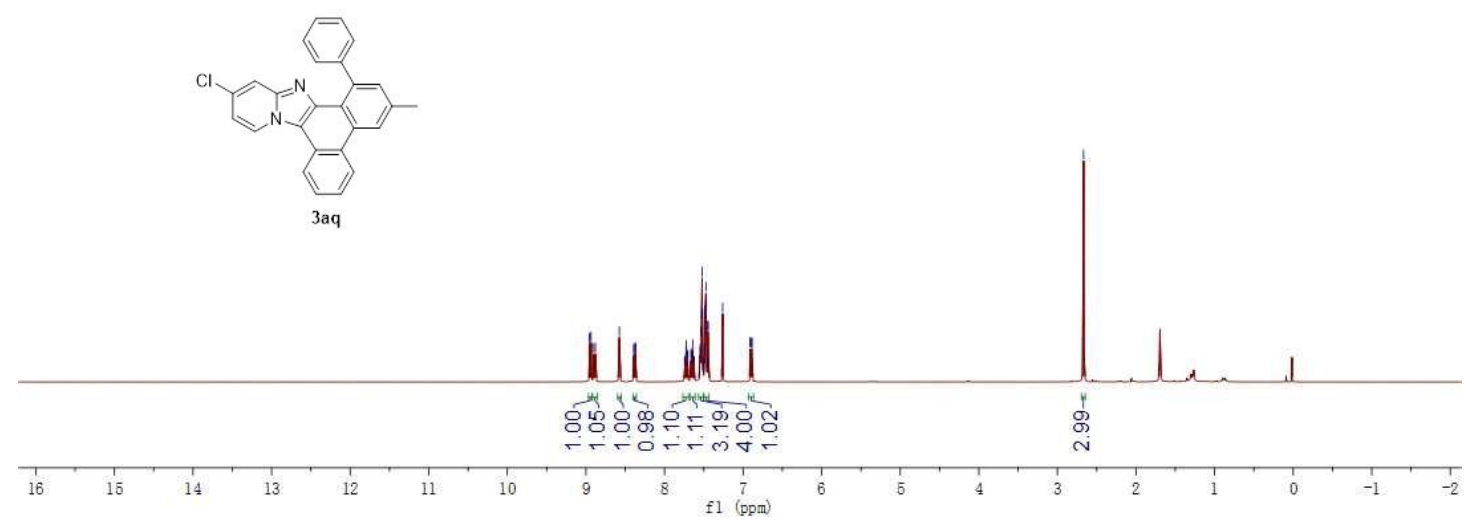



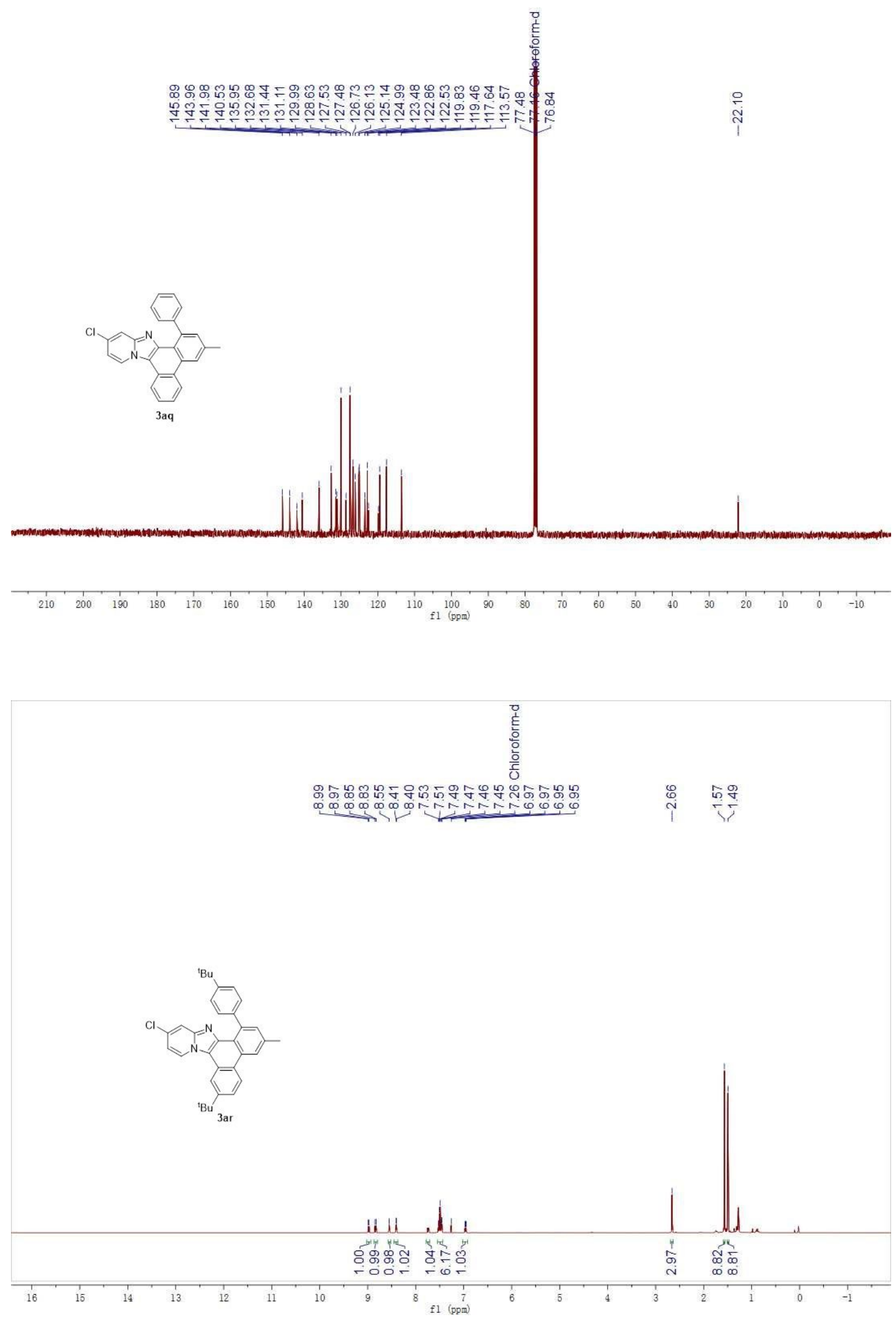


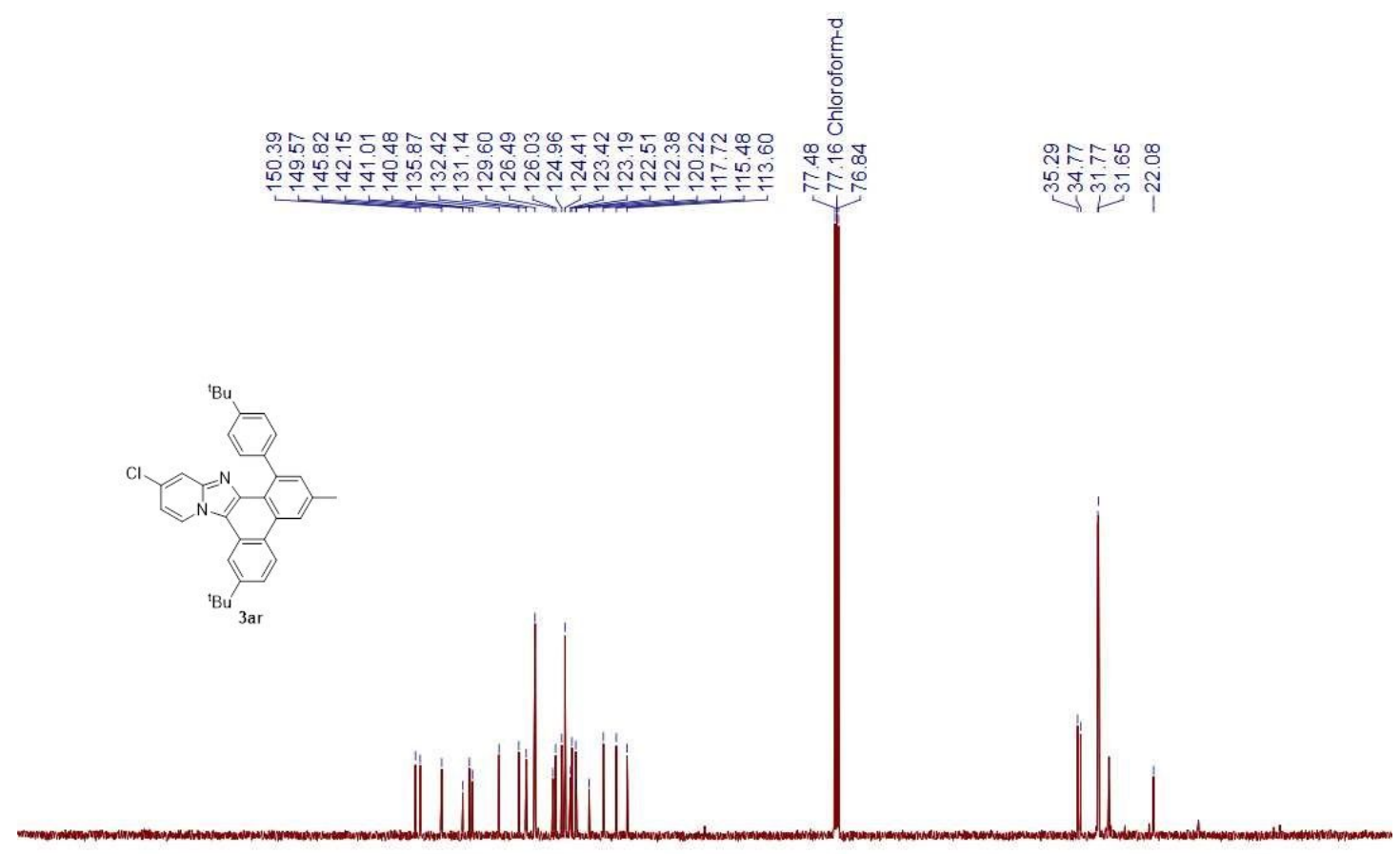

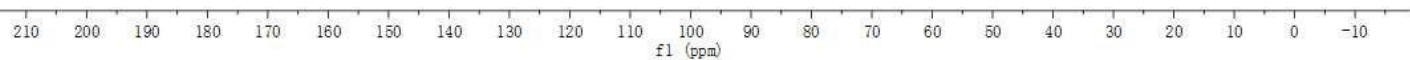

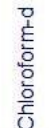

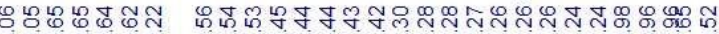

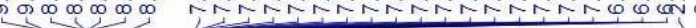
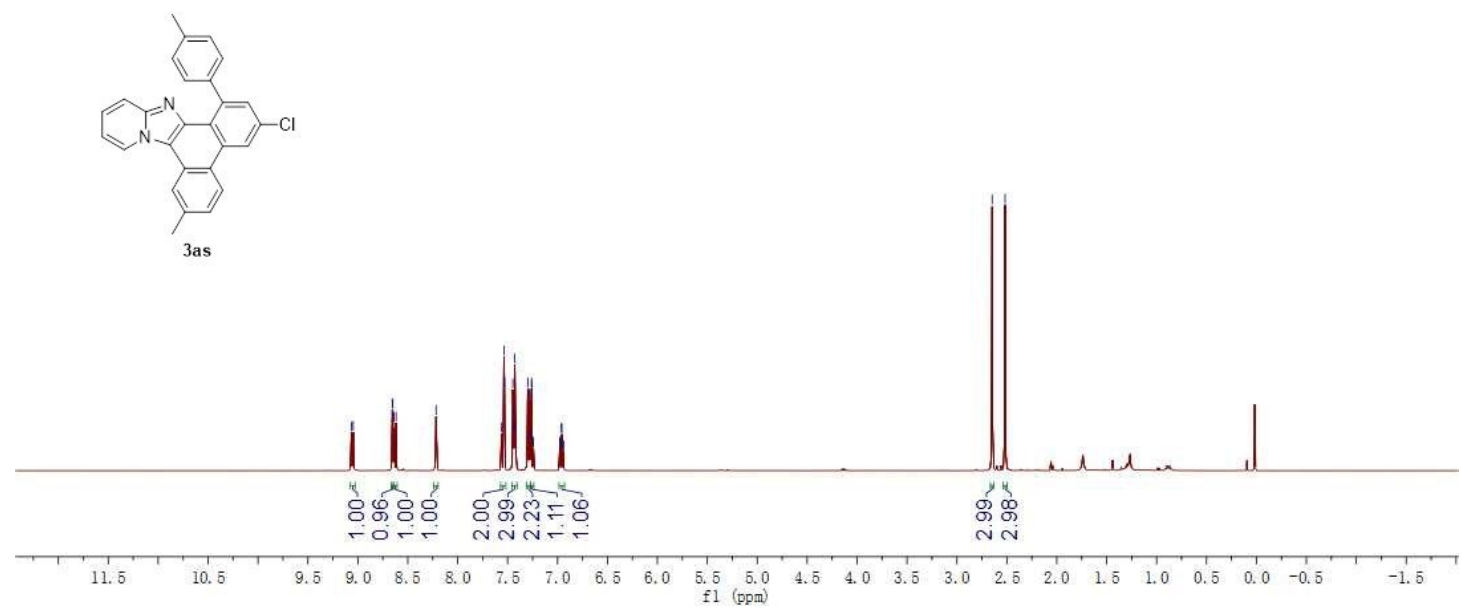

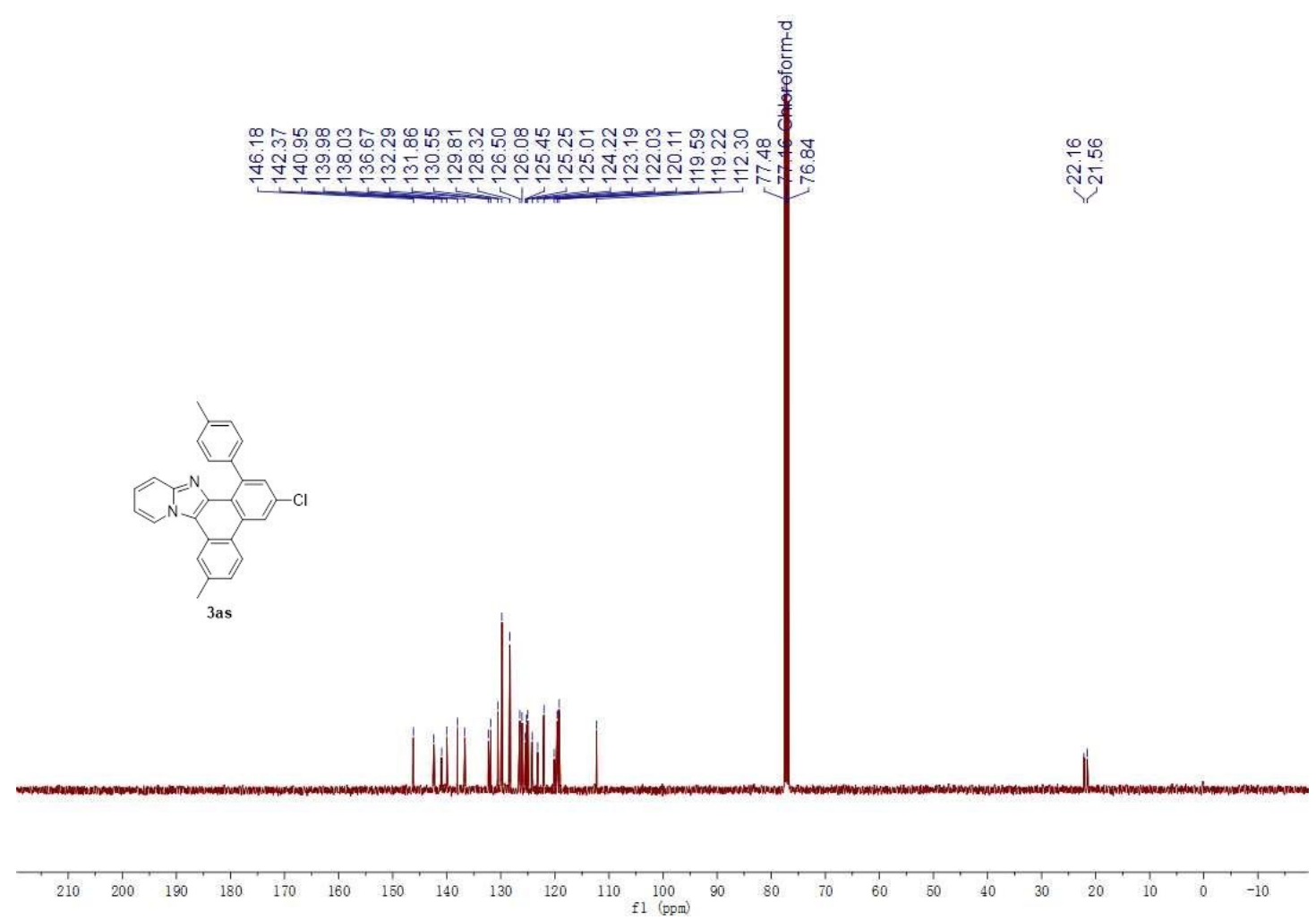

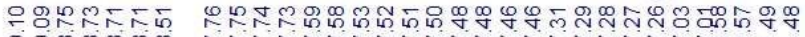

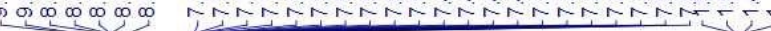

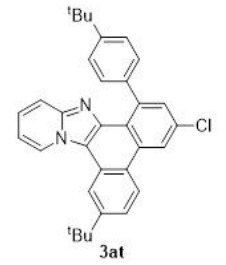

ill this

M

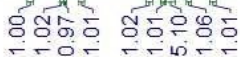

空

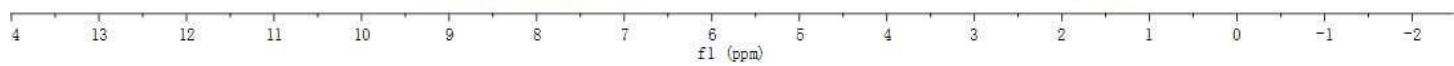




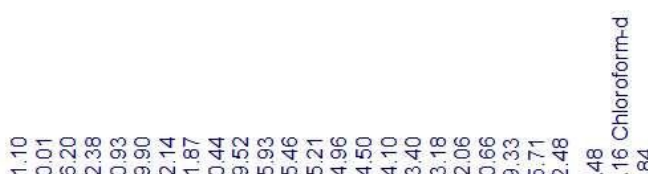

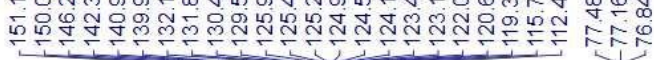

लำ

फलंलूल
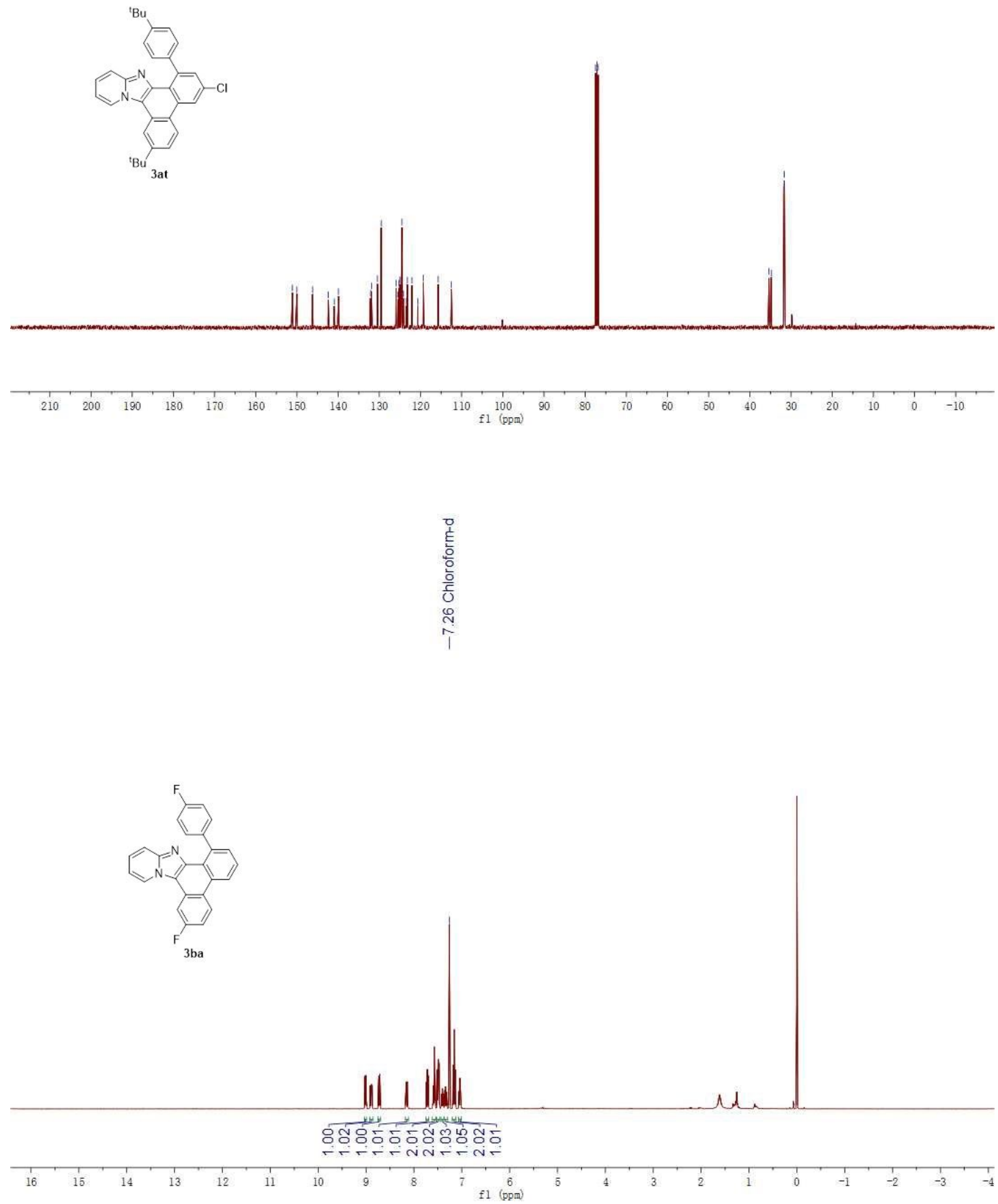

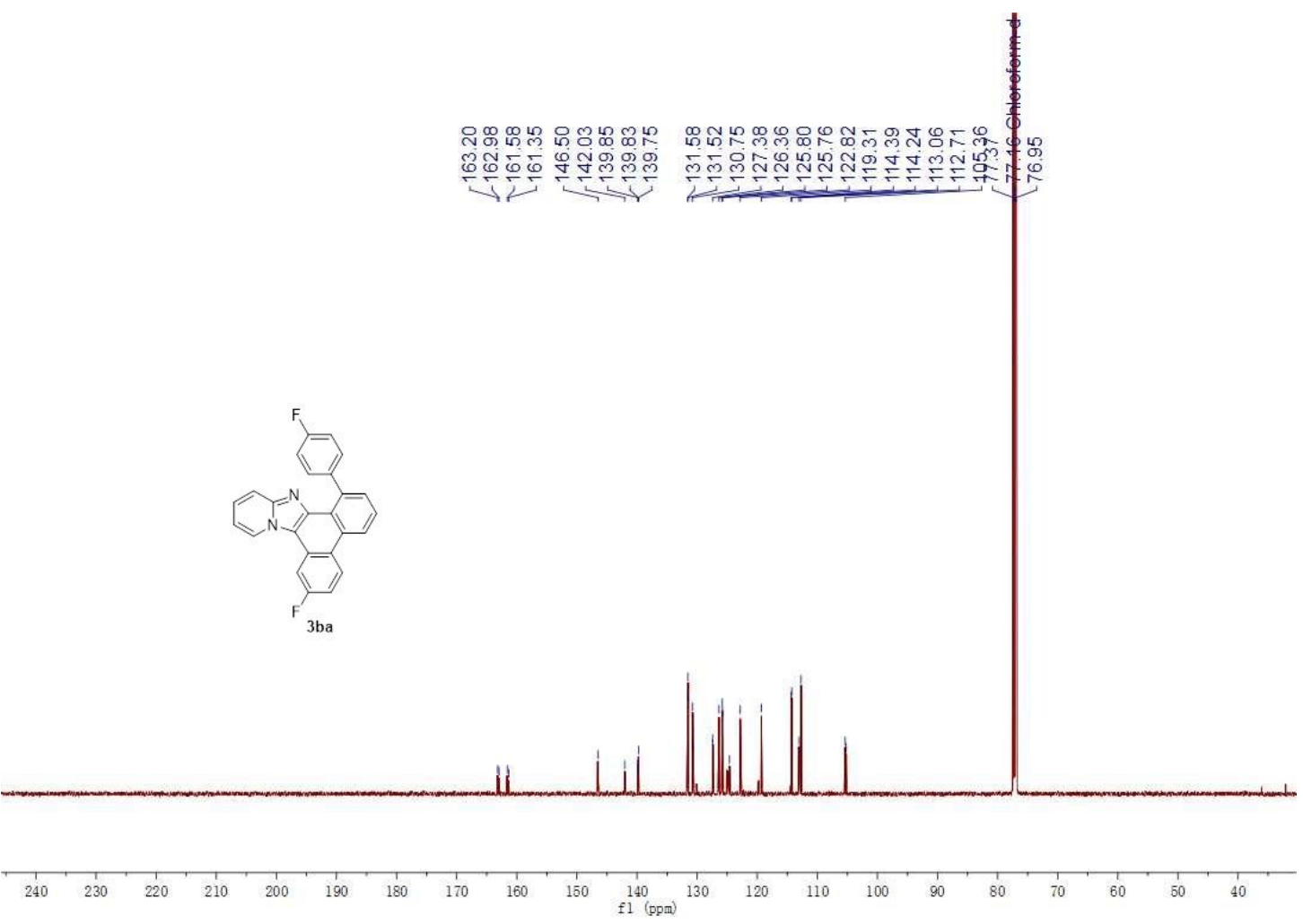

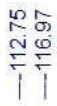
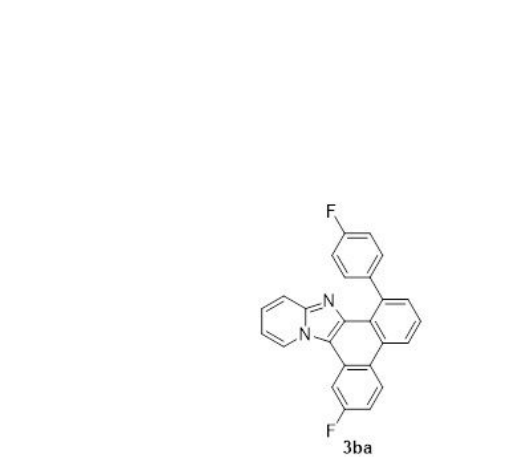

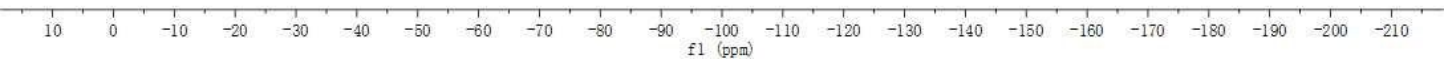




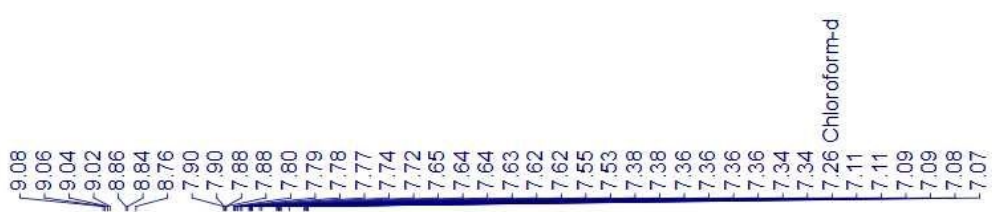

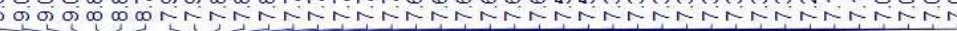
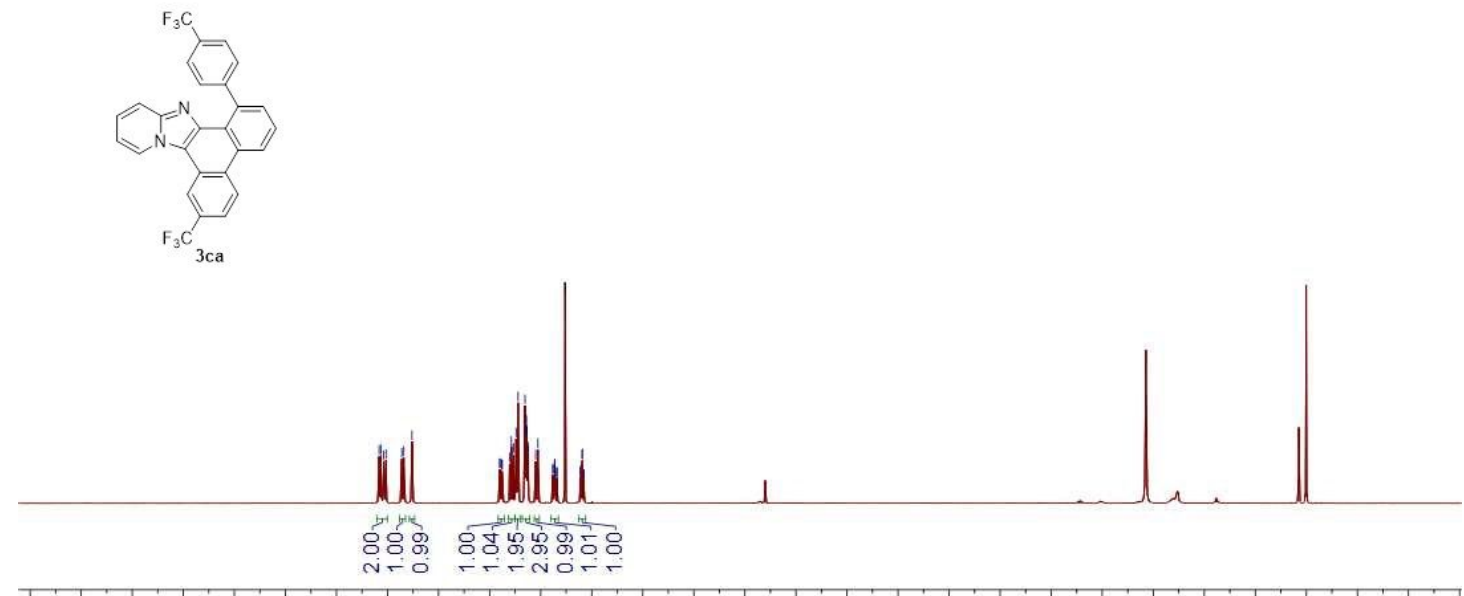

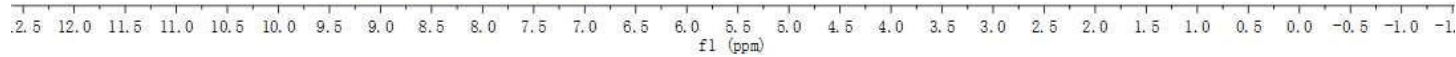
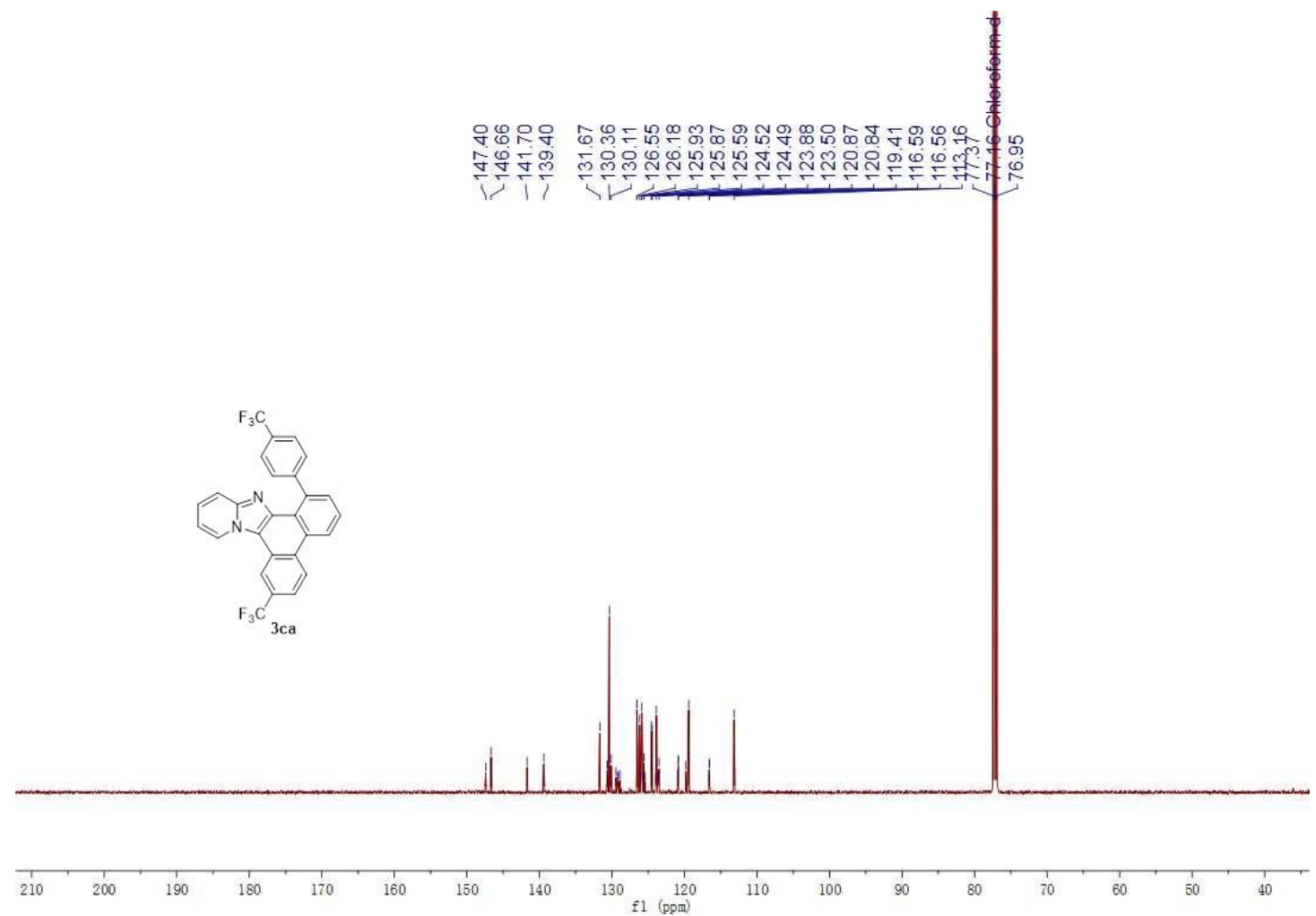


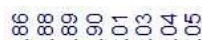

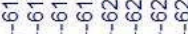
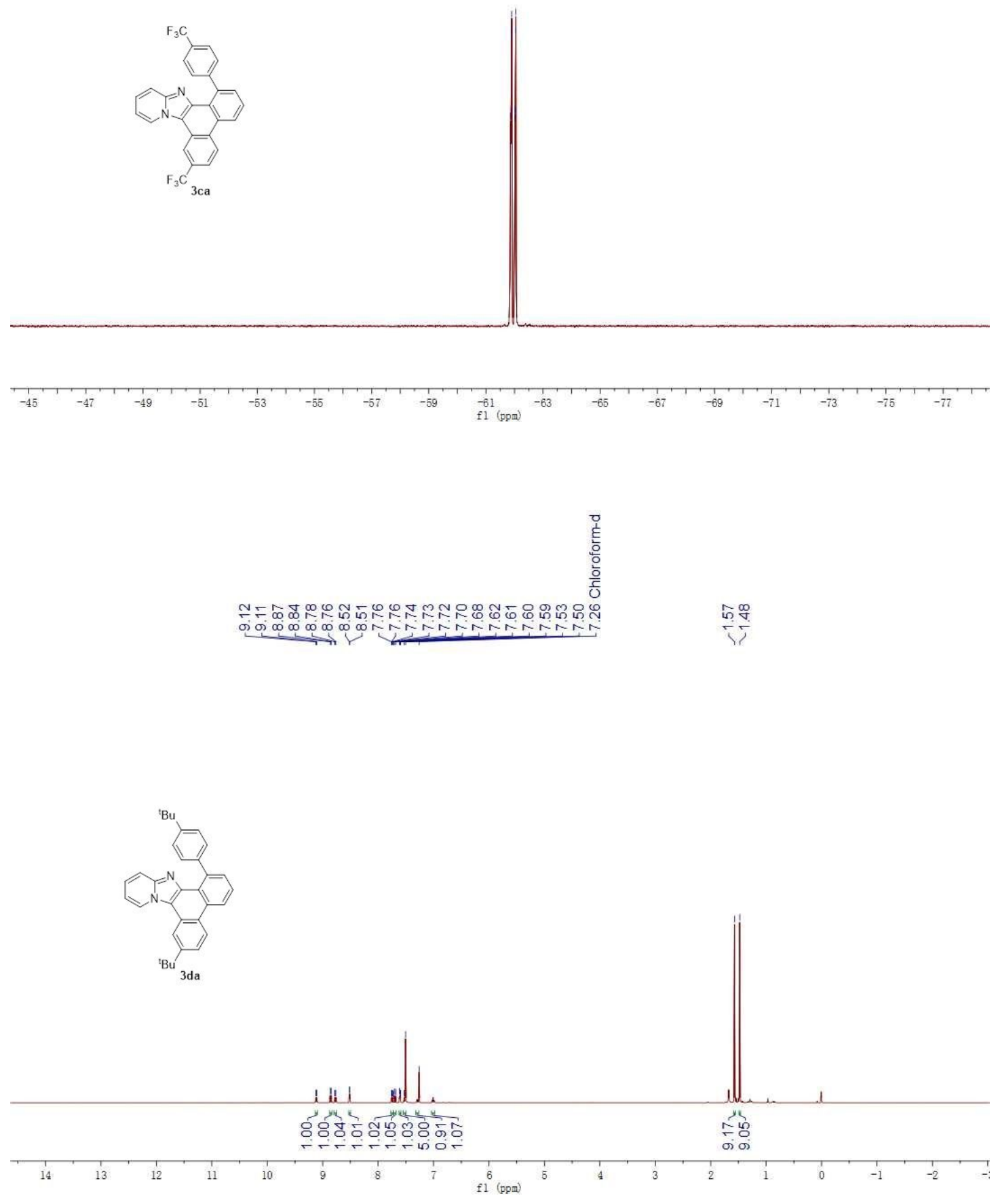

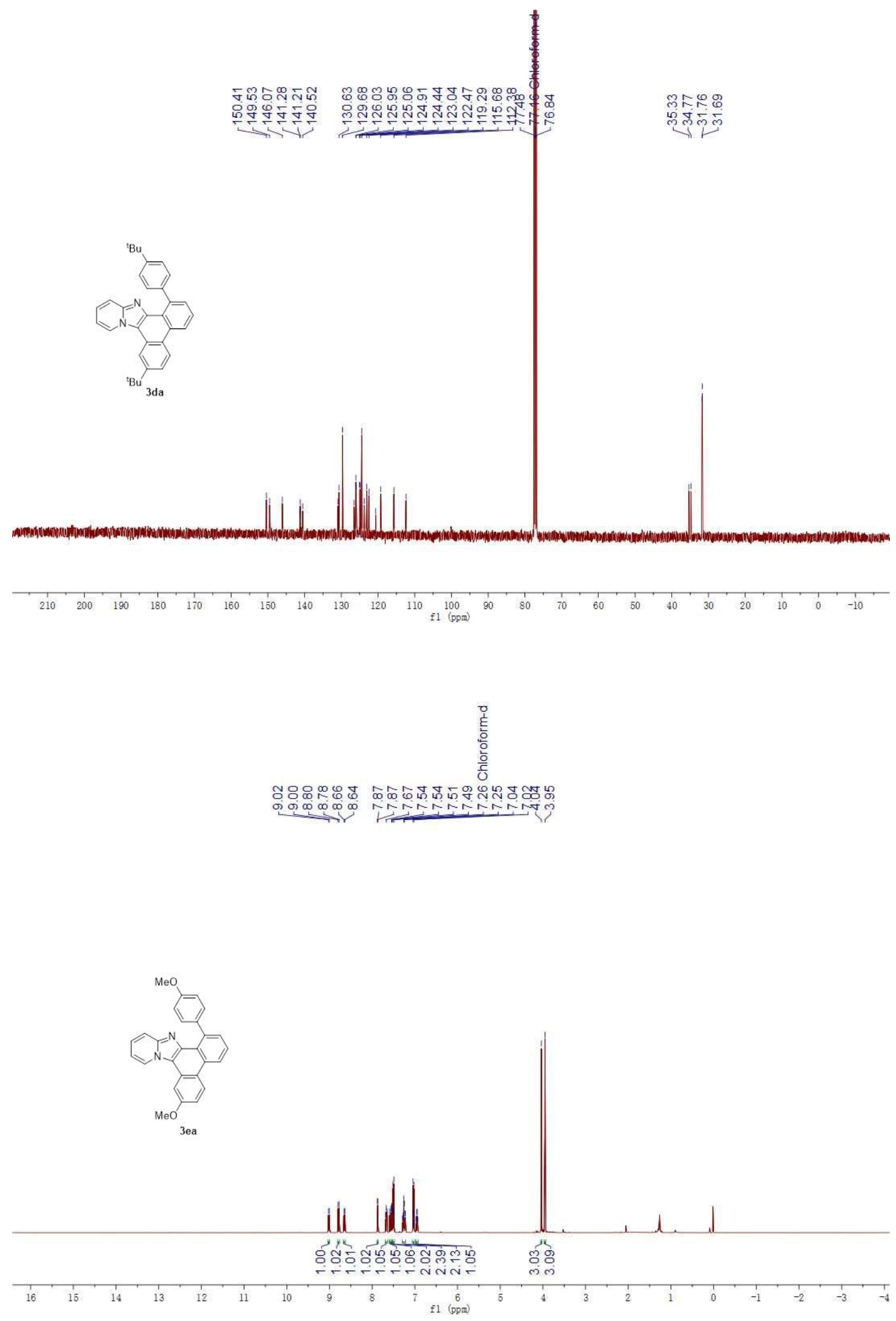


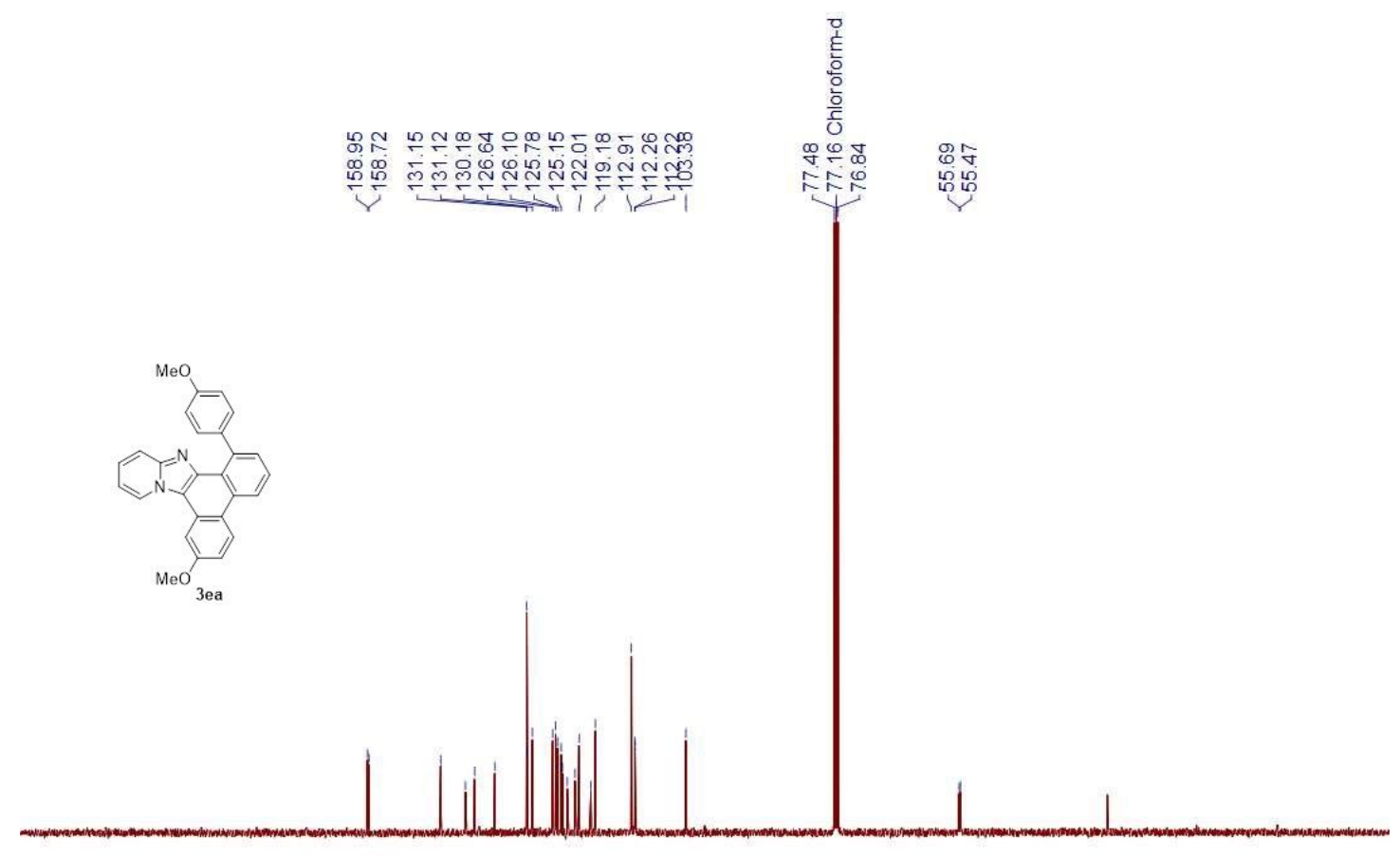

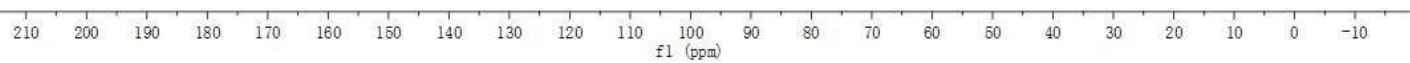

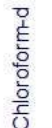

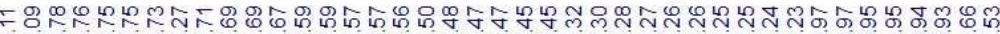

N-N

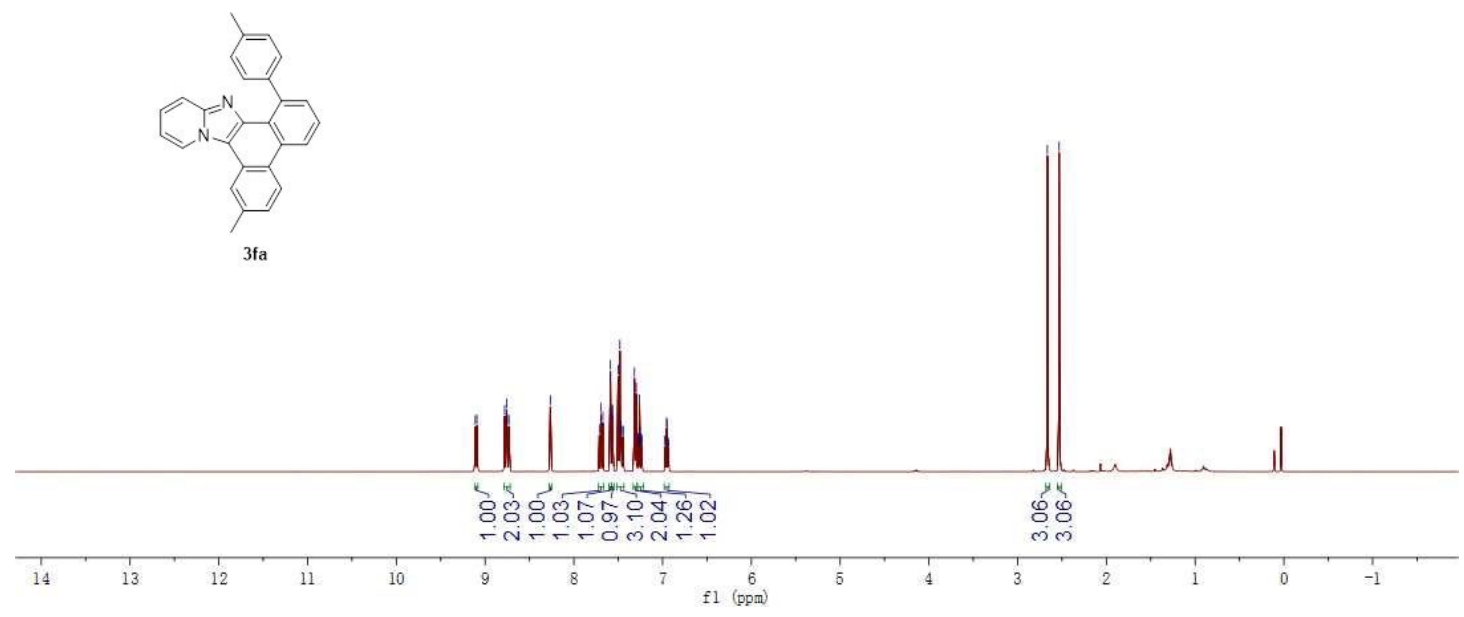



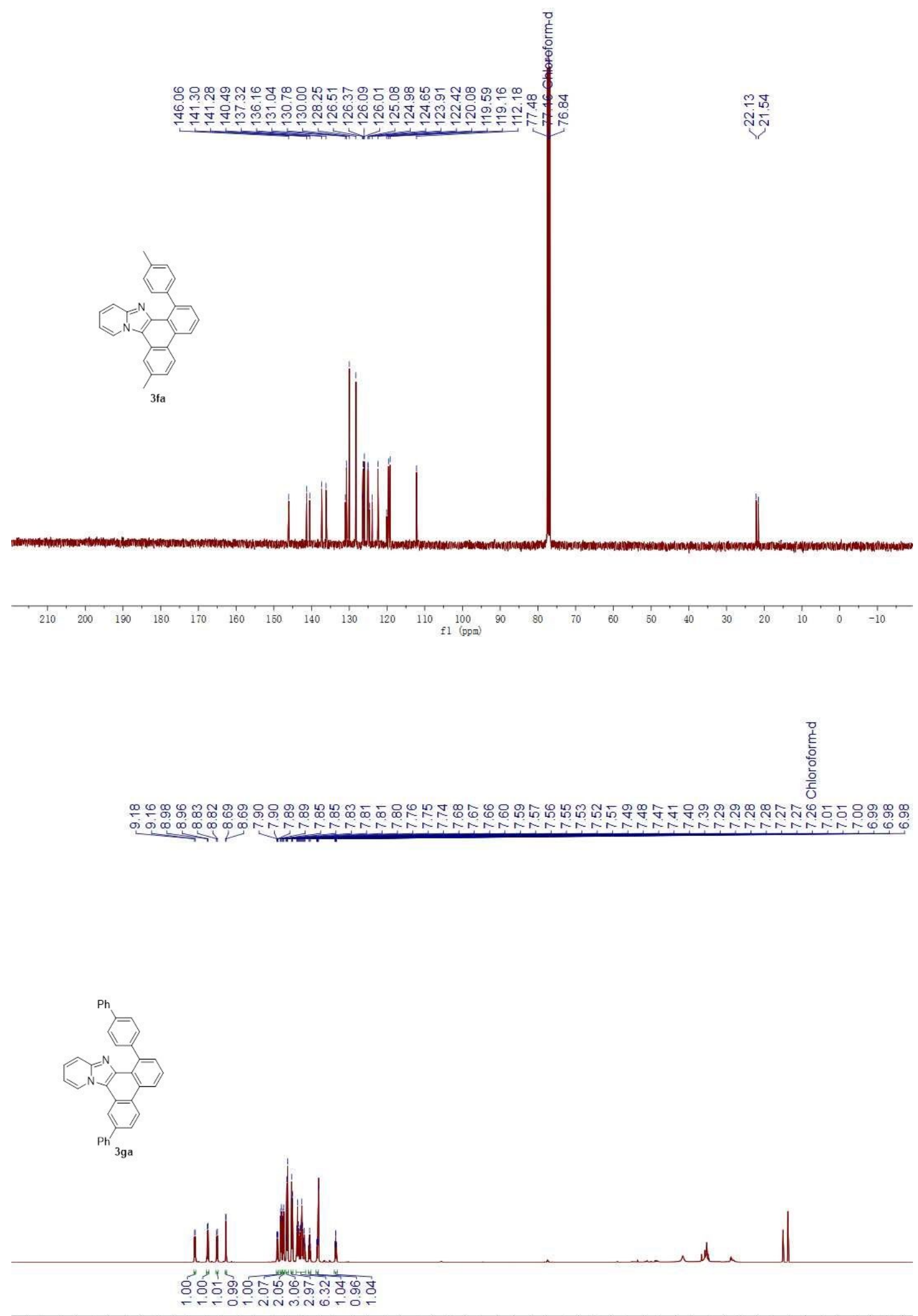

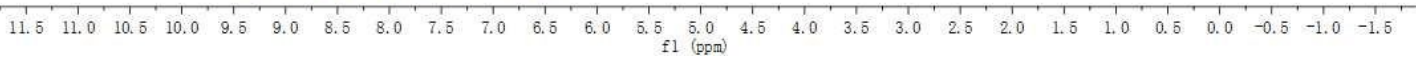



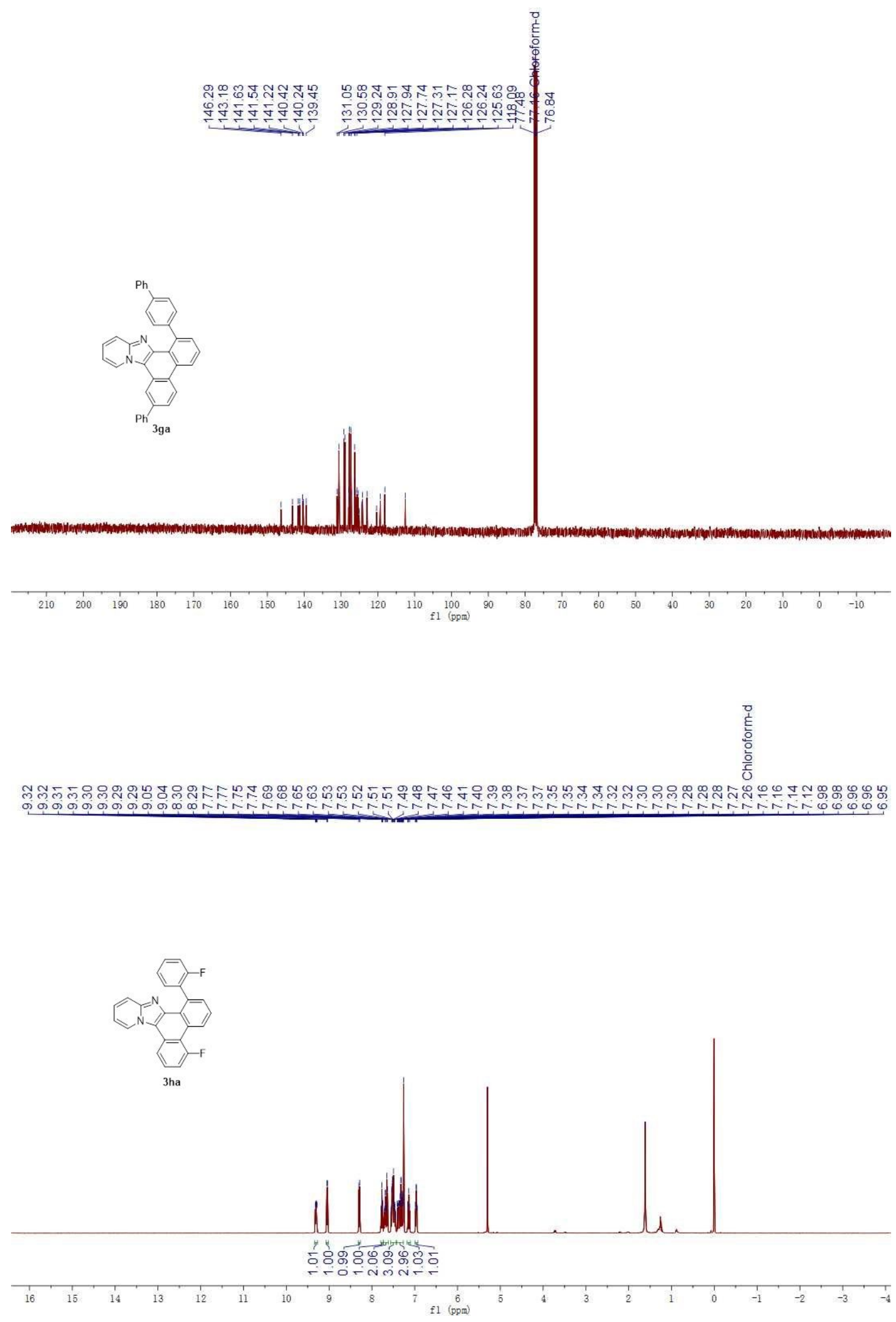


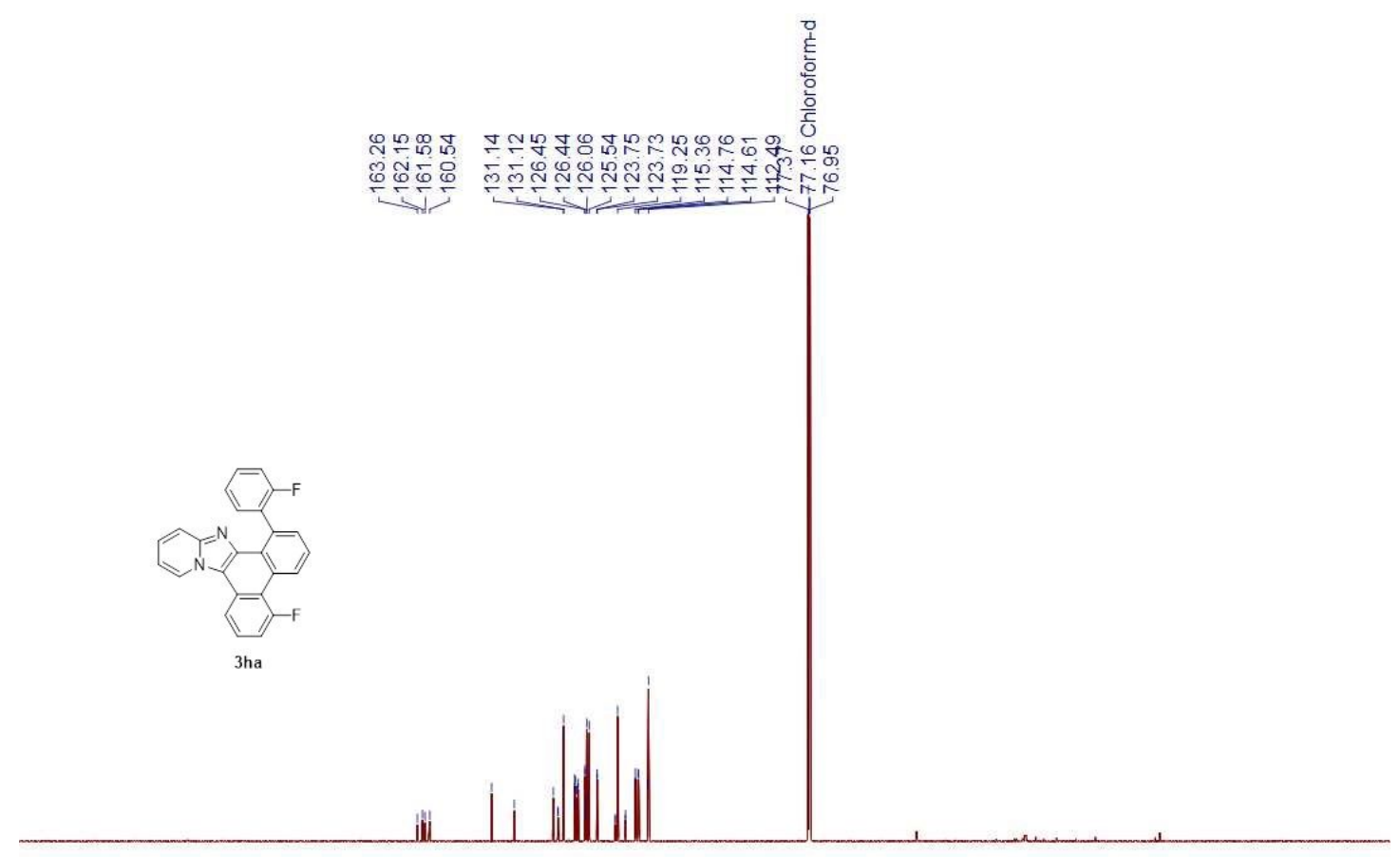

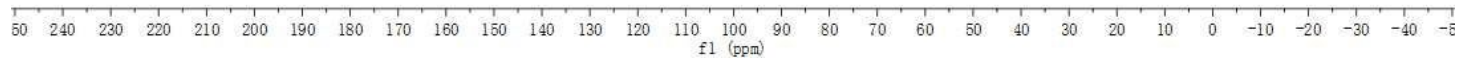

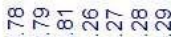

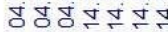

近运

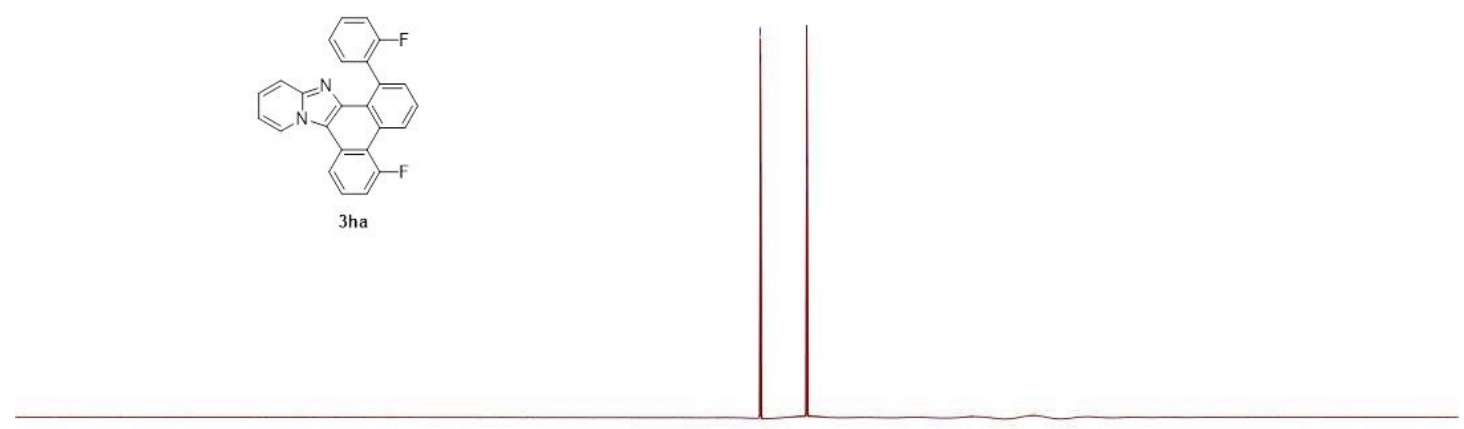

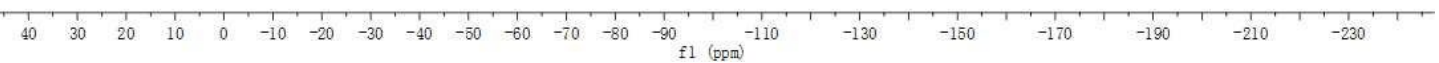




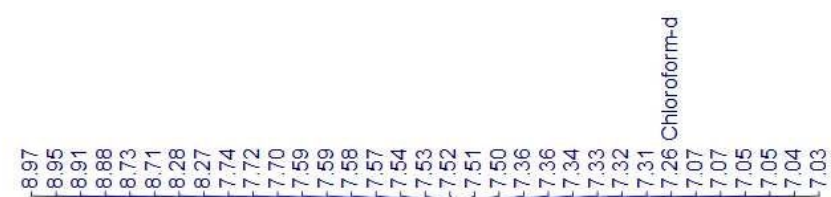

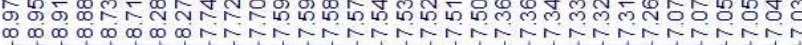
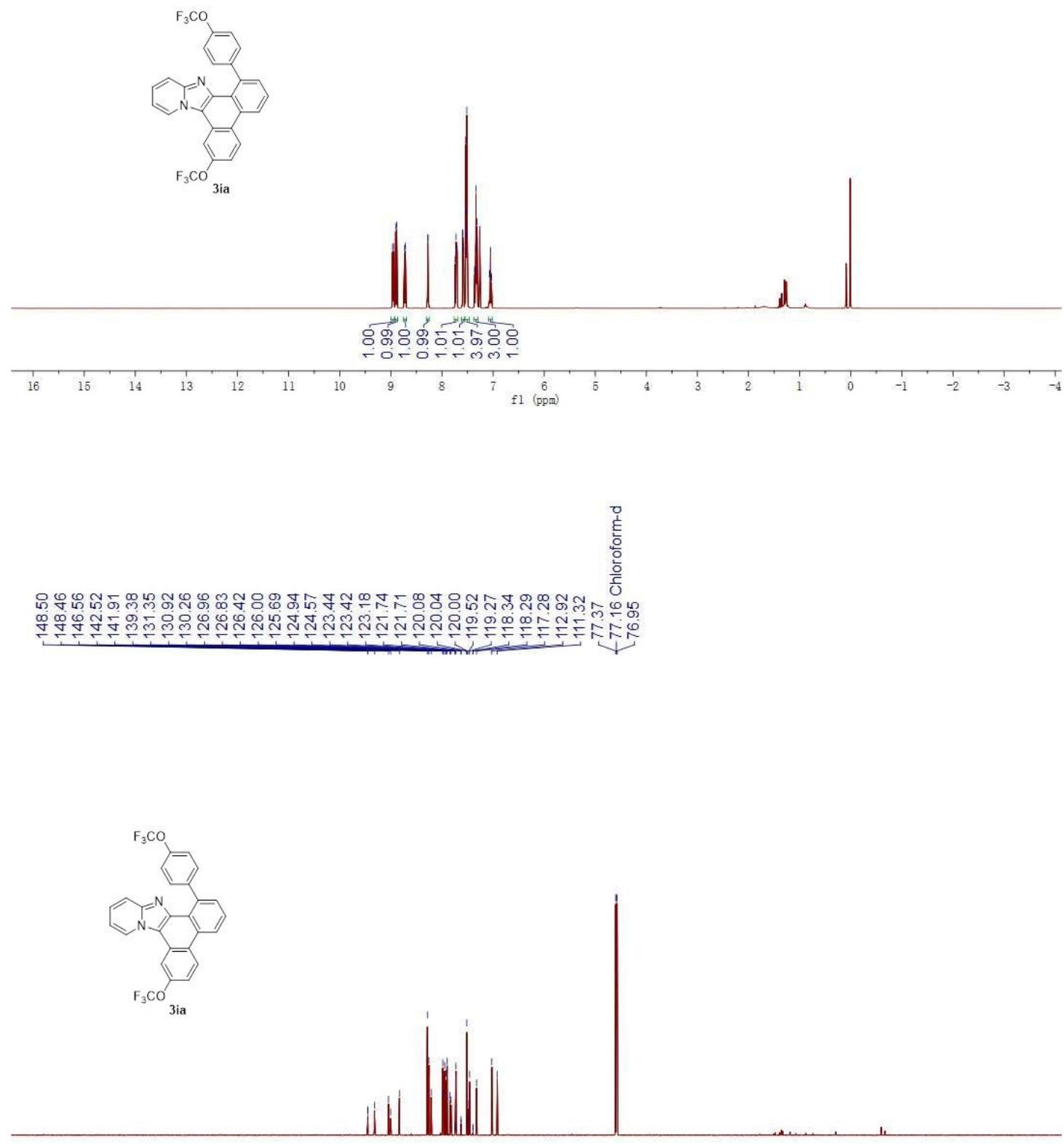

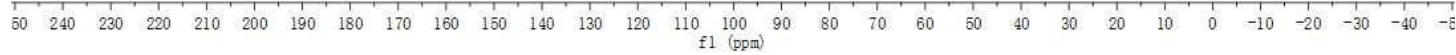



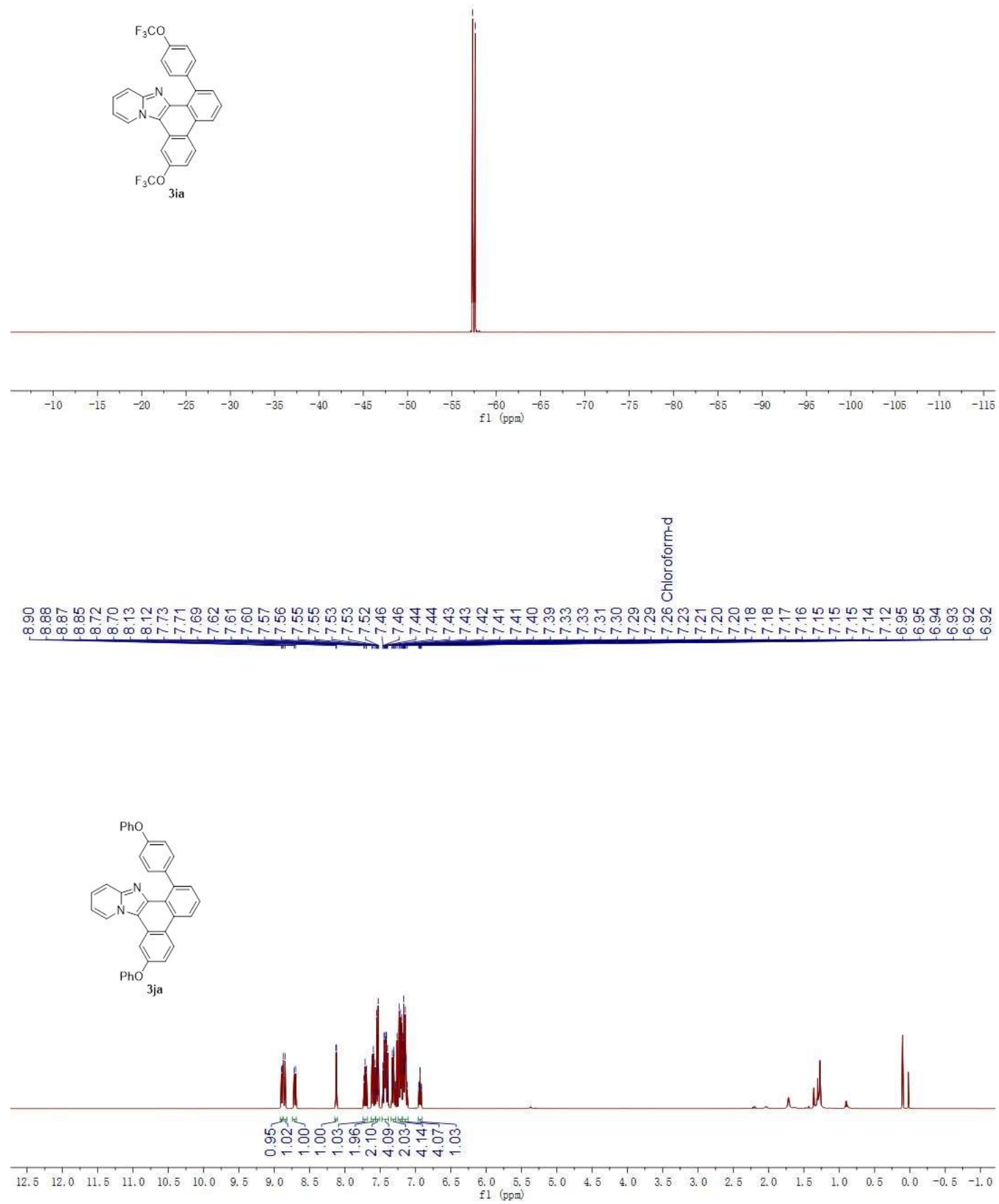


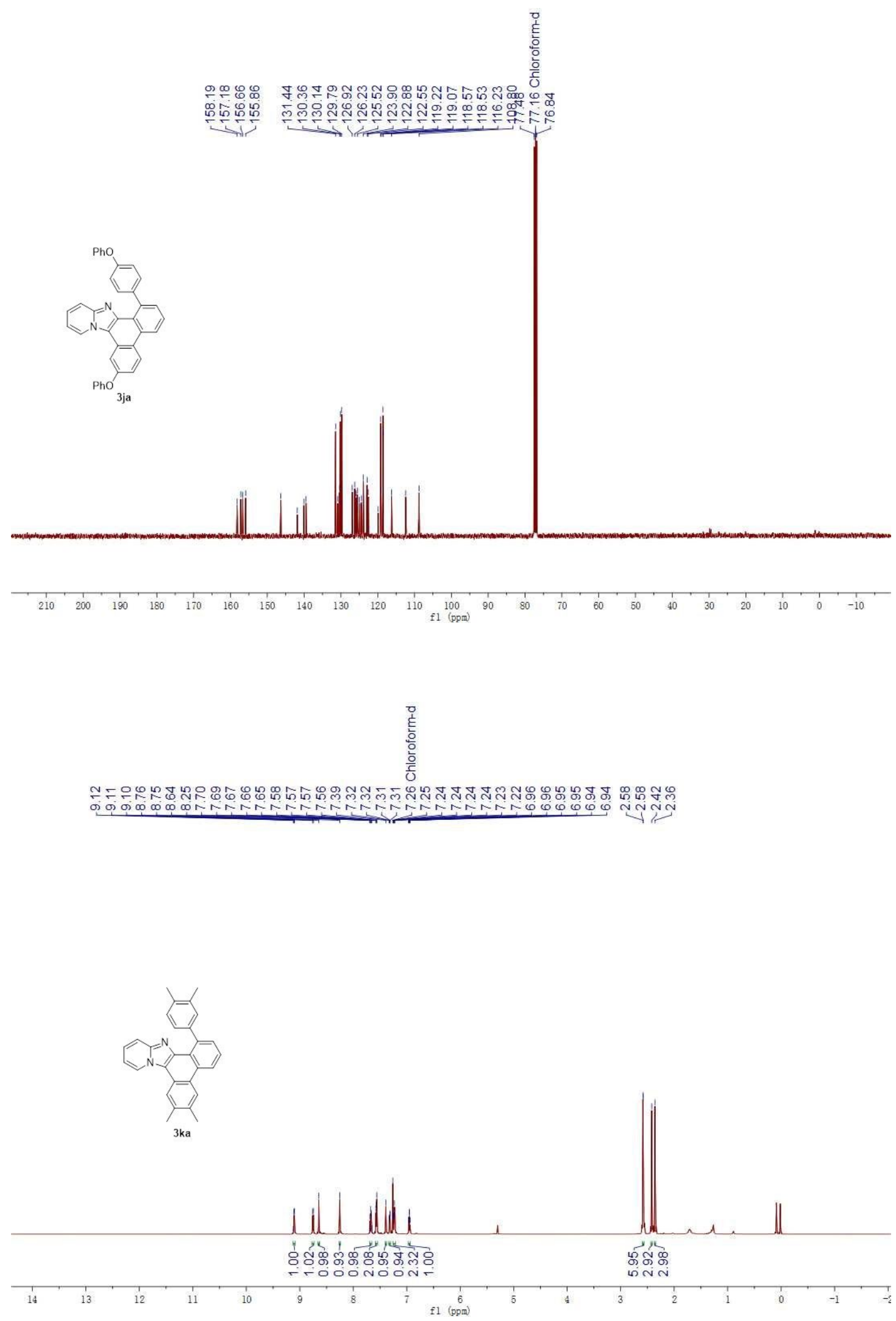




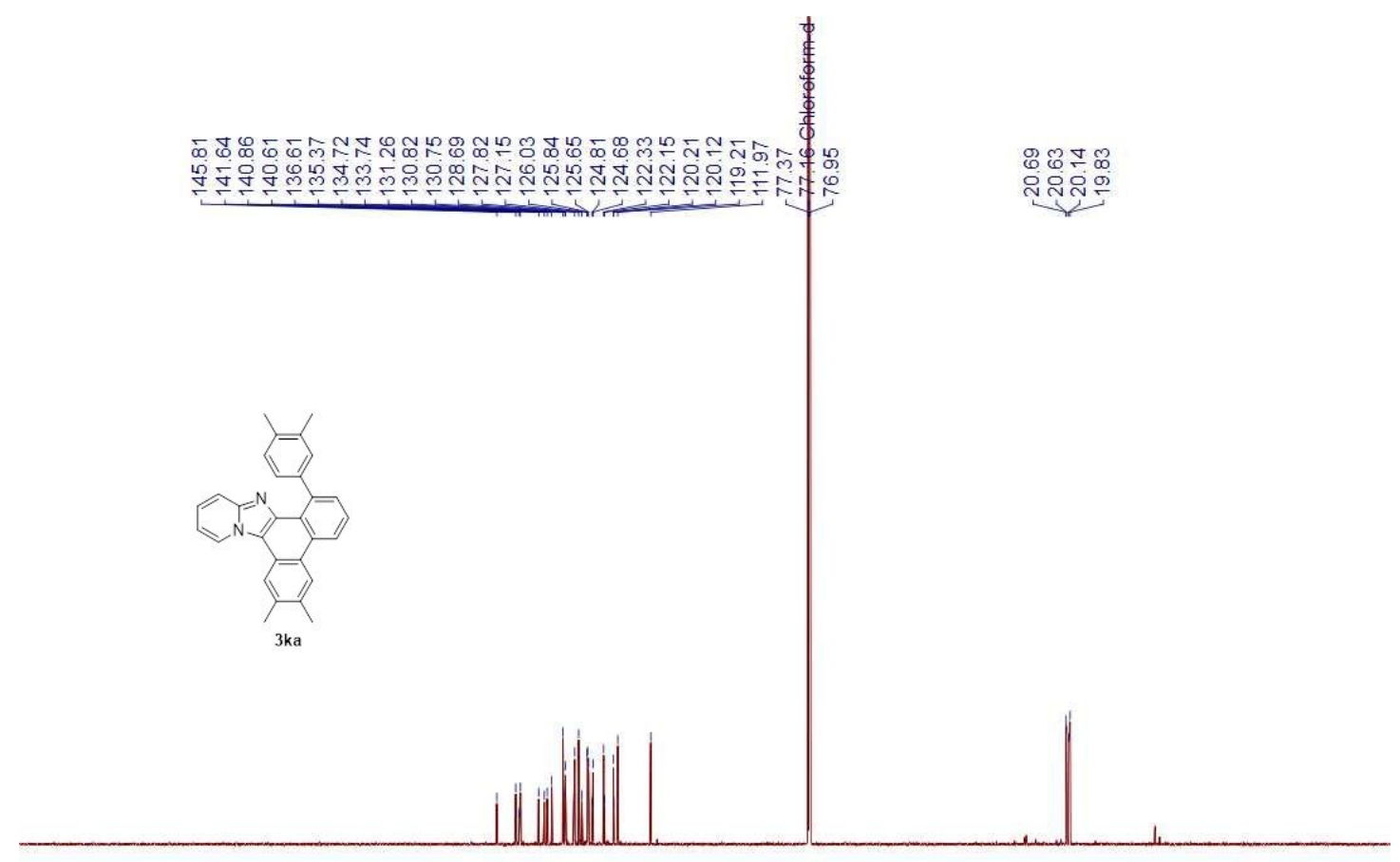

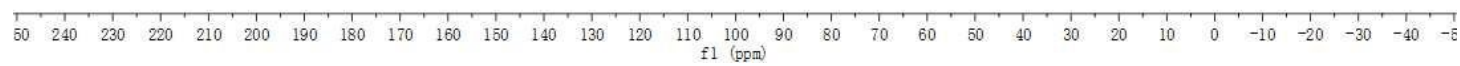

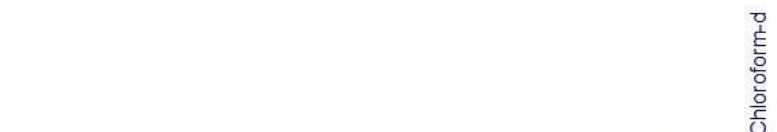

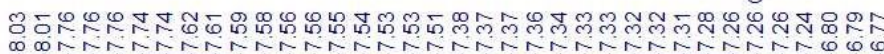

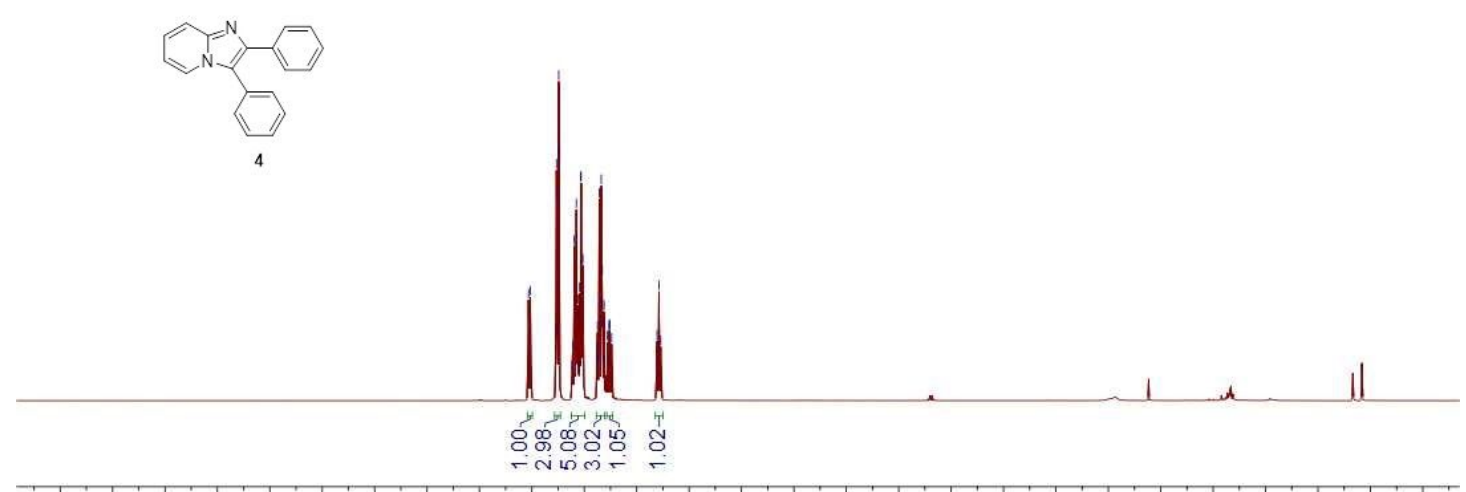

$\begin{array}{llllllllllllllllllllllllllll}12.5 & 12.0 & 11.5 & 11.0 & 10.5 & 10.0 & 9.5 & 9.0 & 8.5 & 8.0 & 7.5 & 7.0 & 6.5 & 6.0 & 5.5 & 5.0 & 4.5 & 4.0 & 3.5 & 3.0 & 2.5 & 2.0 & 1.5 & 1.0 & 0.5 & 0.0 & -0.5\end{array}$ 

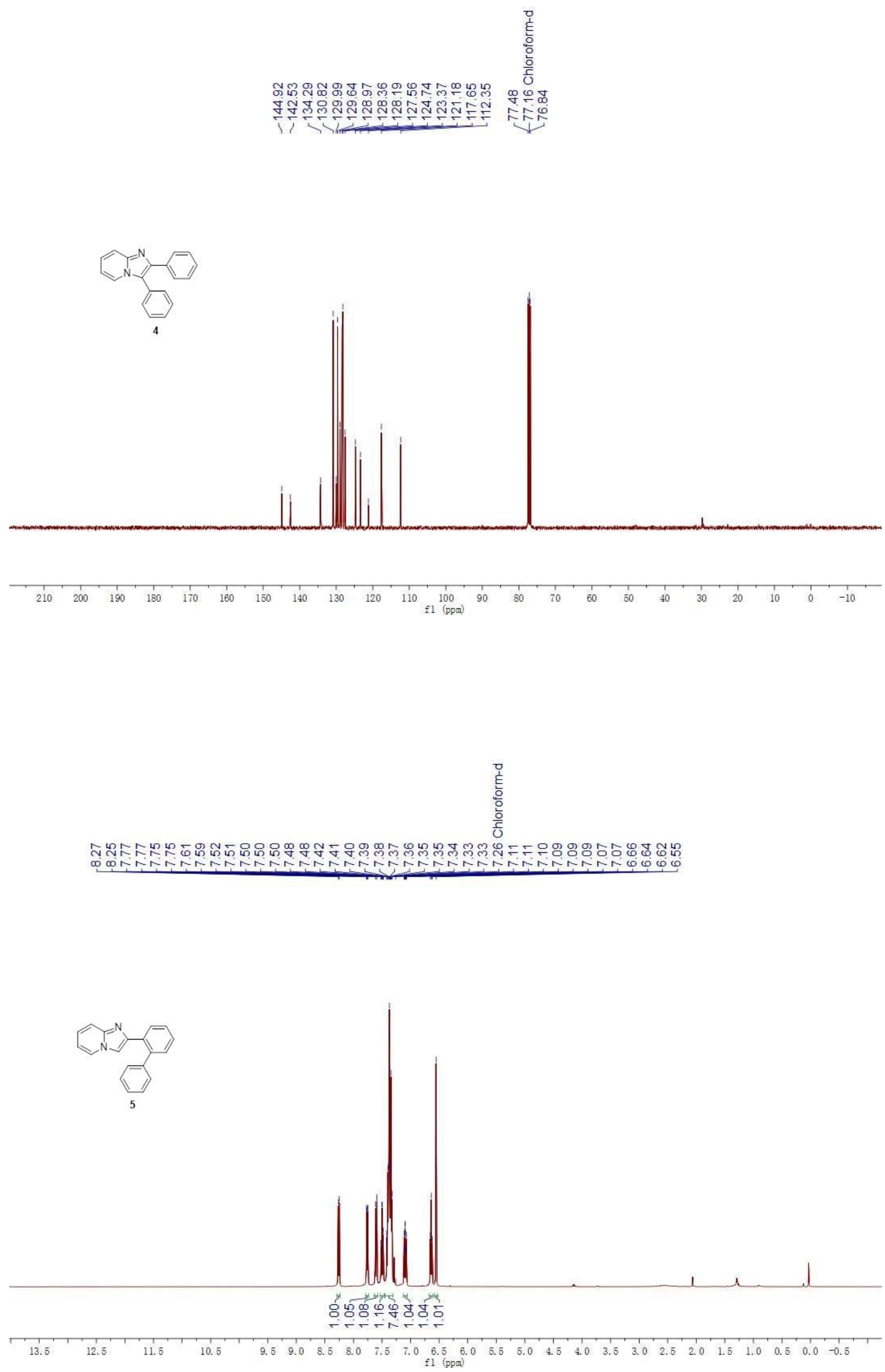

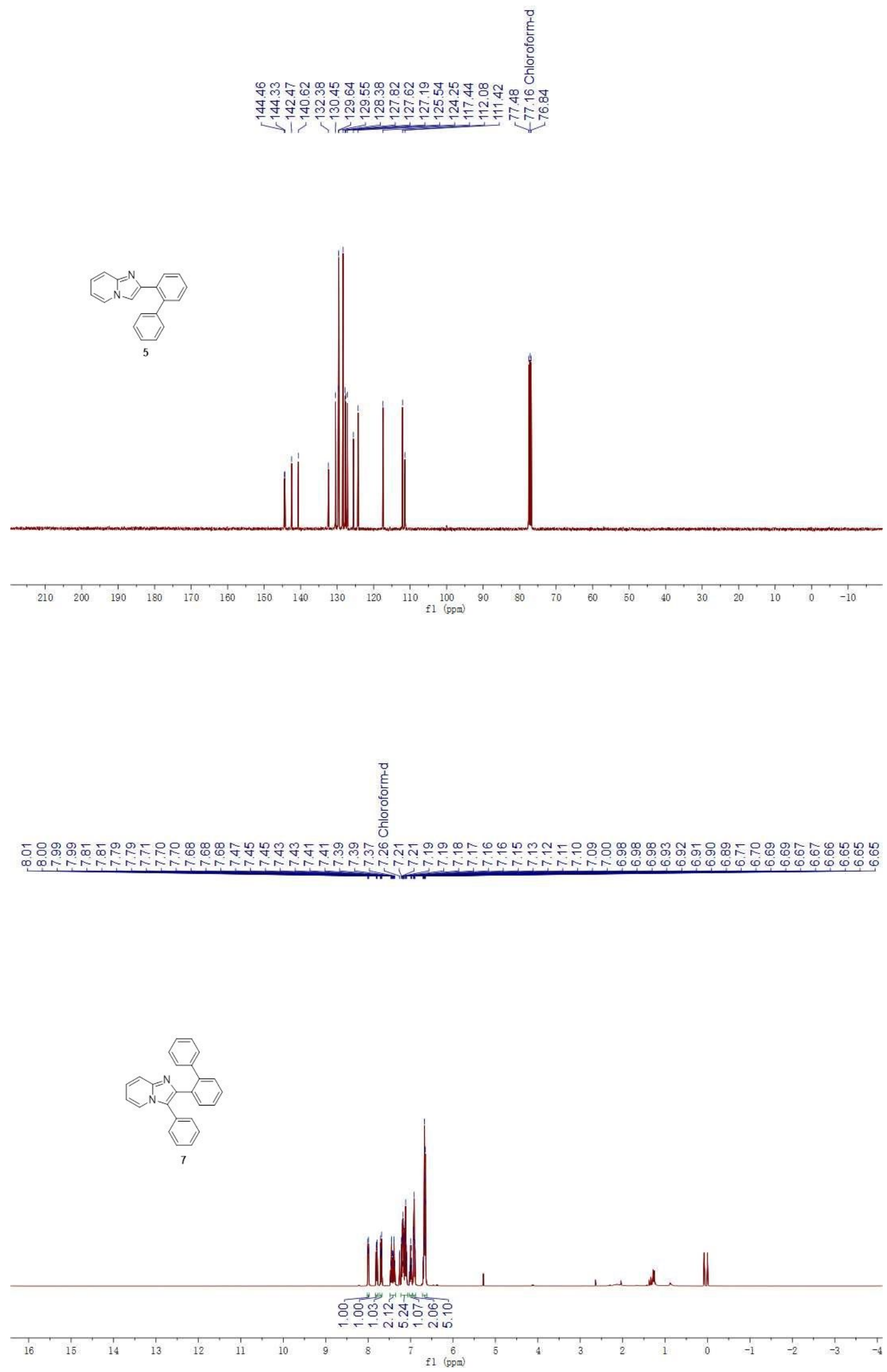


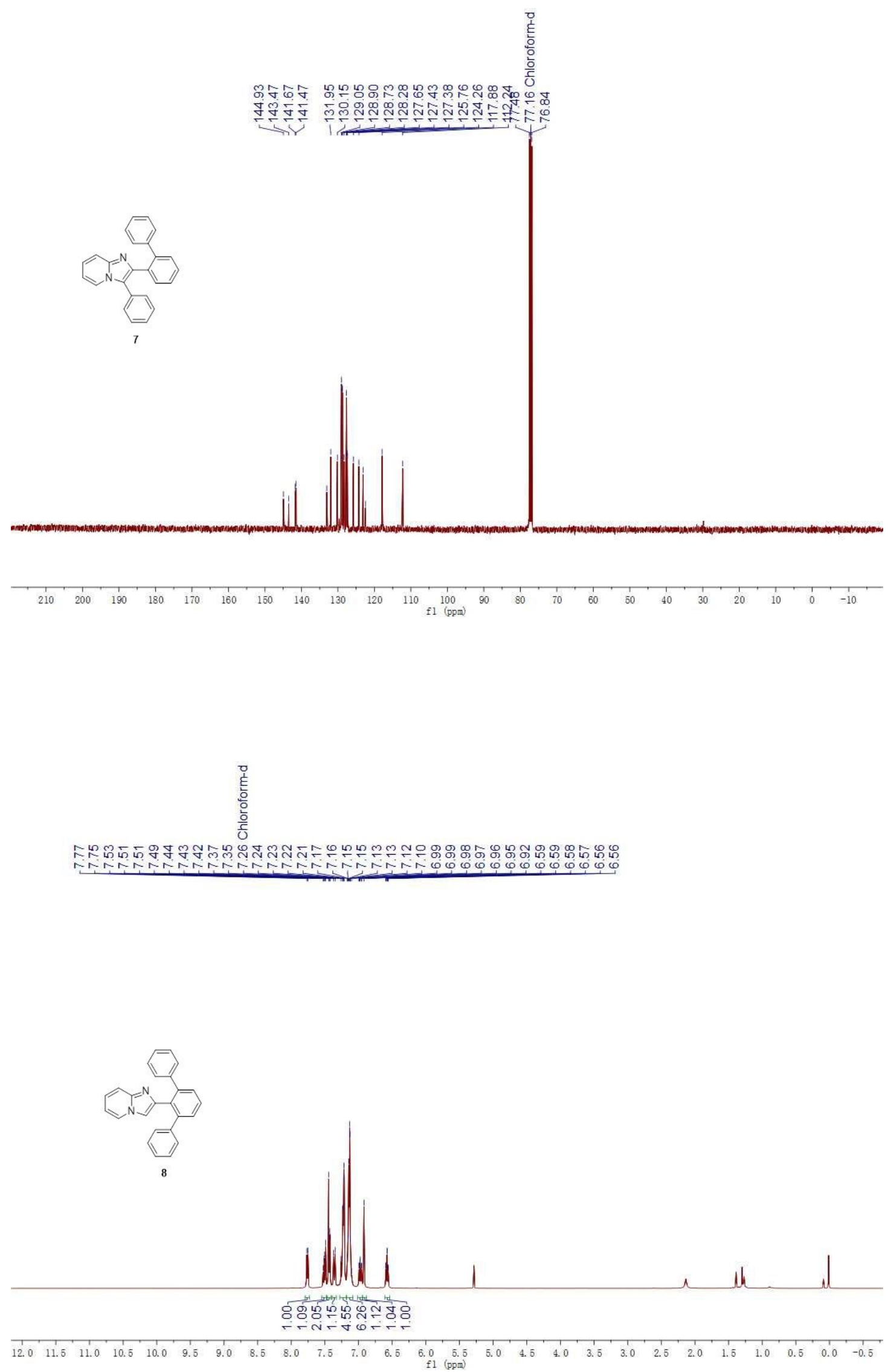



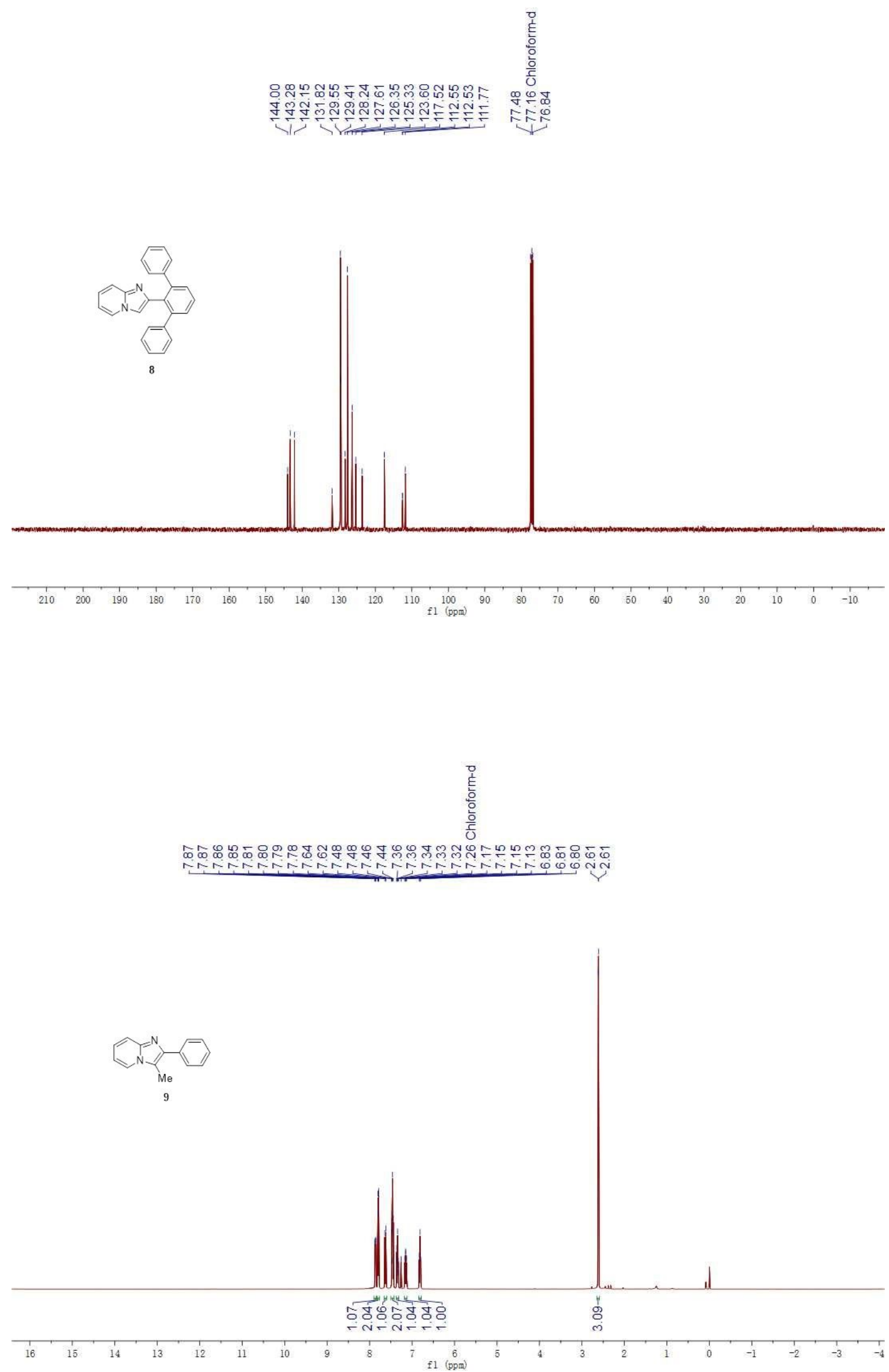

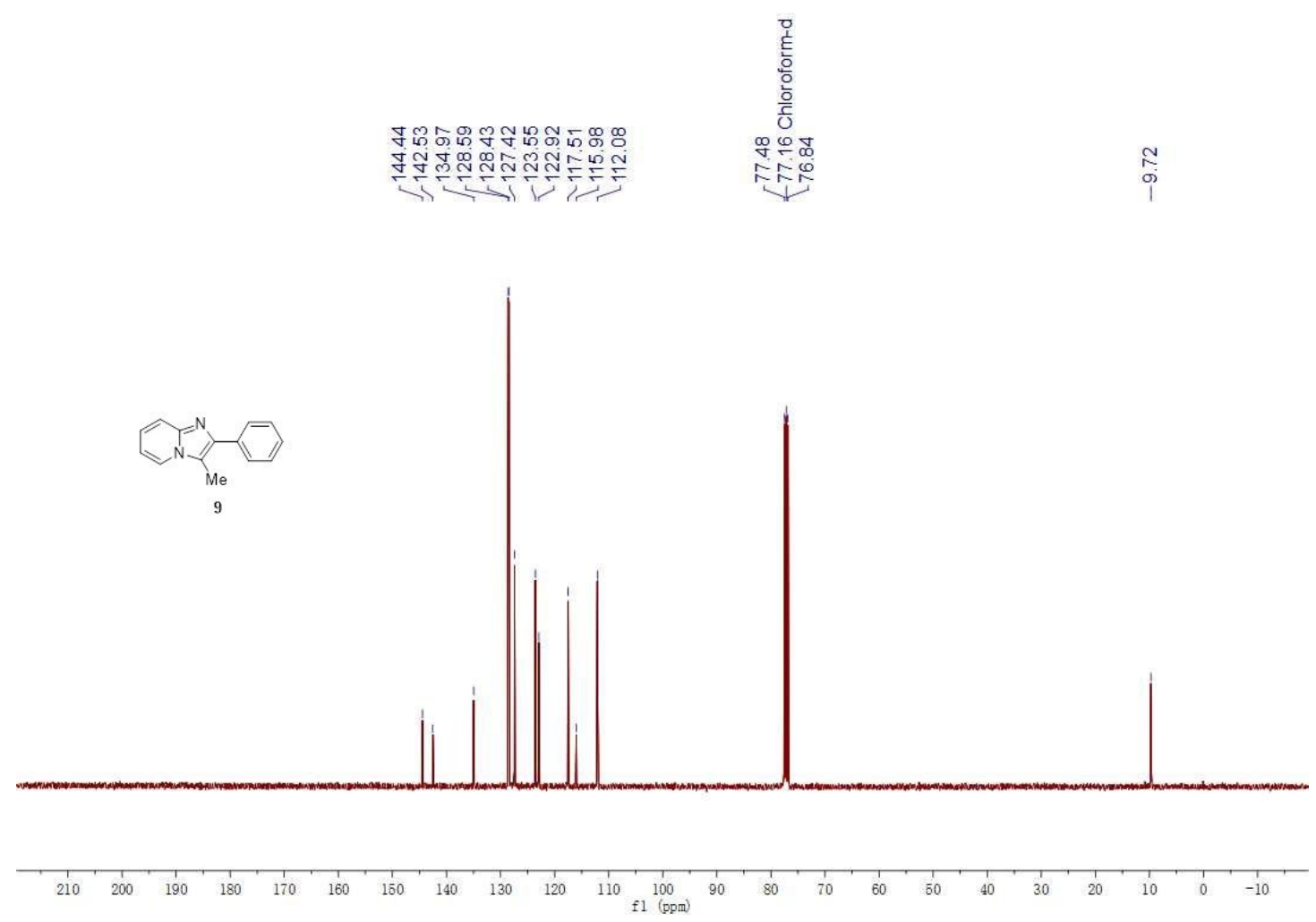

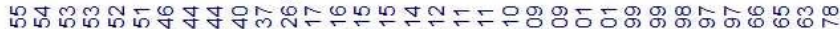

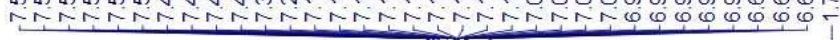

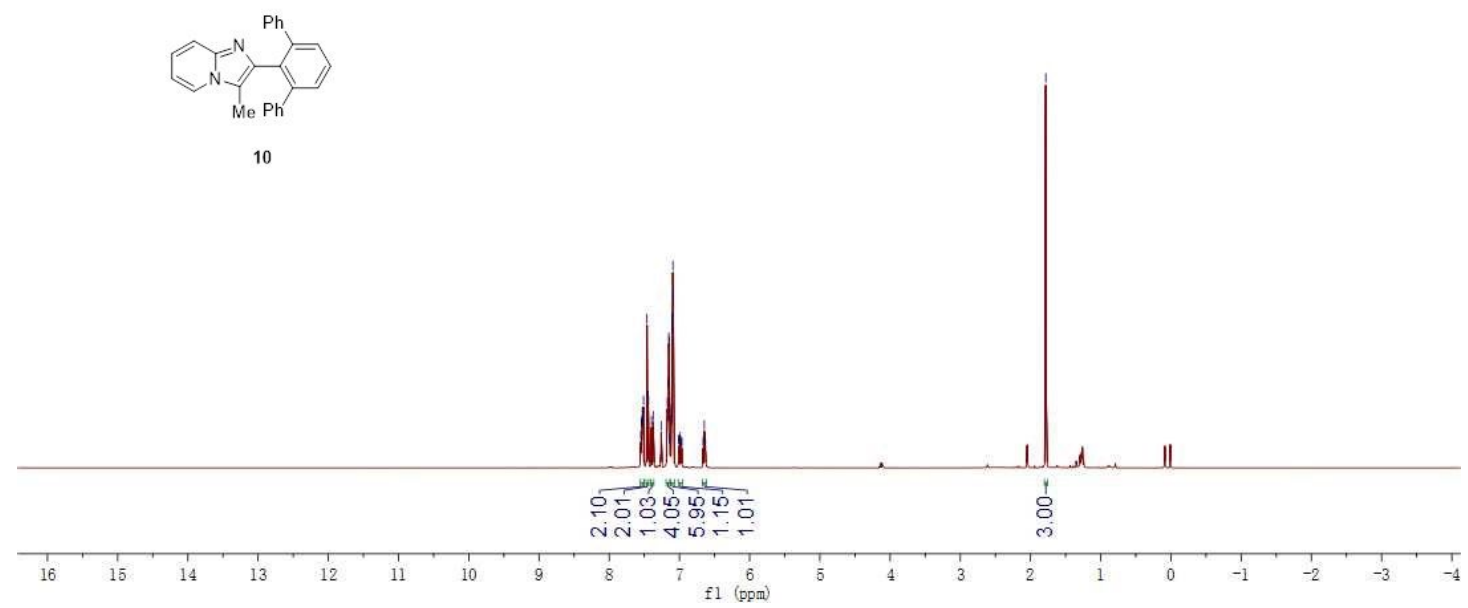



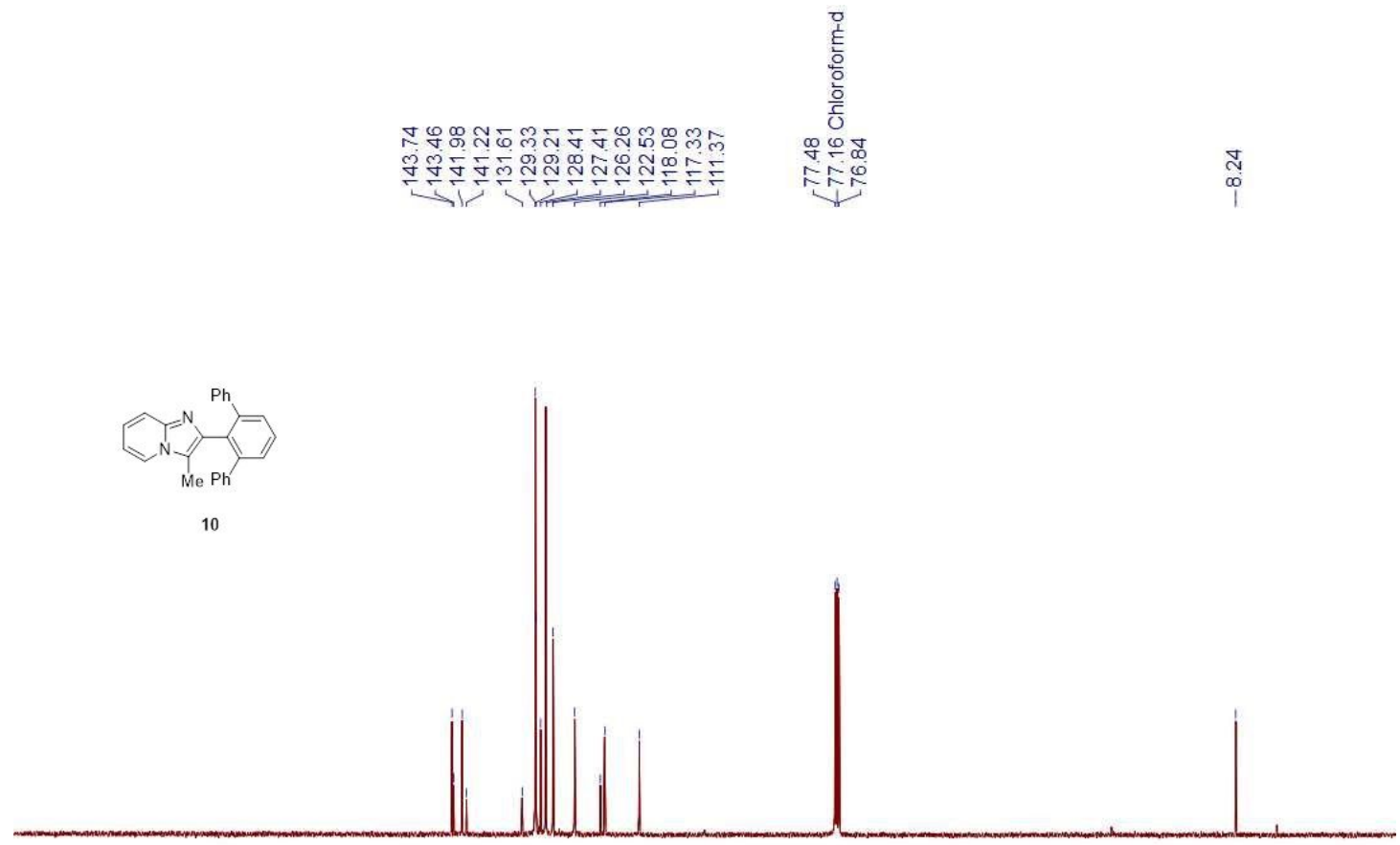

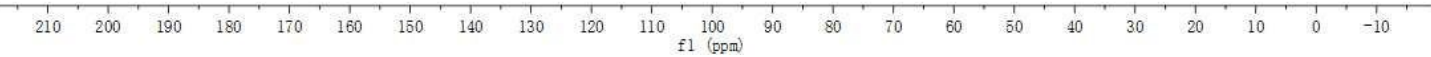<smiles>CO[As]</smiles>

N.

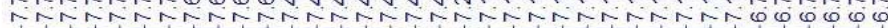

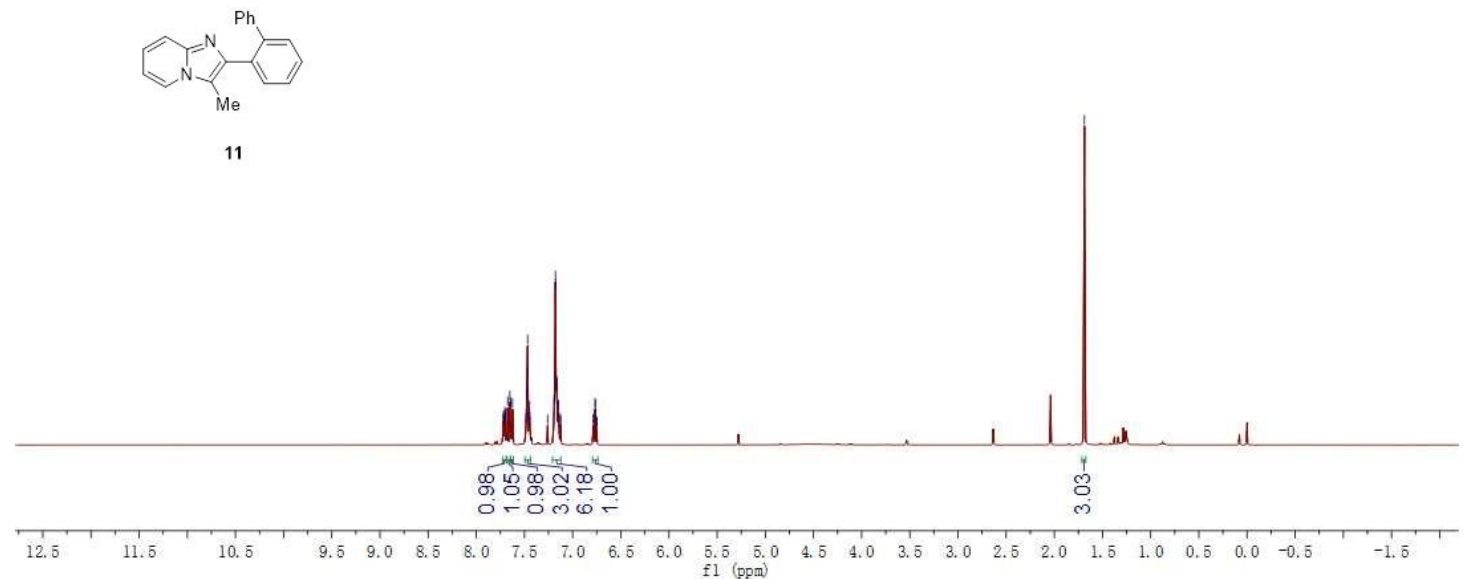




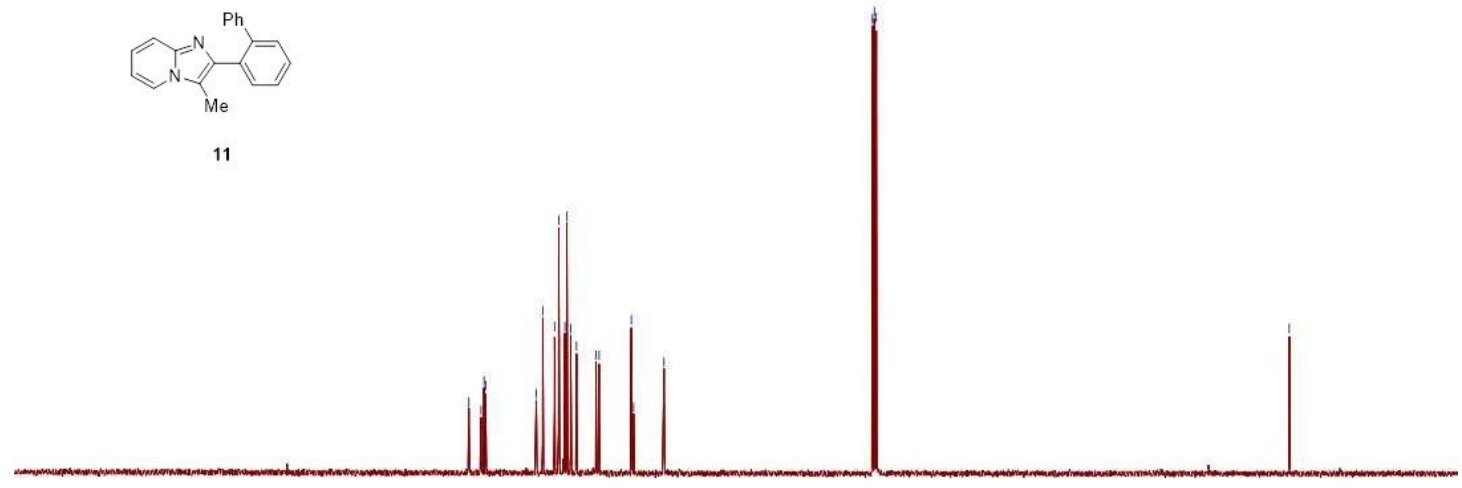

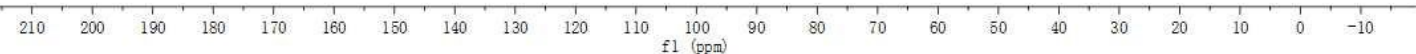

